\title{
Multi-Higgs boson production and unitarity in vector-boson fusion at future hadron colliders
}

\author{
Wolfgang Kilian, ${ }^{1, *}$ Sichun Sun, ${ }^{2,3, \dagger}$ Qi-Shu Yan, ${ }^{4,5}$ Xiaoran Zhao, ${ }^{6,8}$ and Zhijie Zhao ${ }^{1,5, \uparrow}$ \\ ${ }^{1}$ Department of Physics, University of Siegen, 57072 Siegen, Germany \\ ${ }^{2}$ Department of Physics and INFN, Sapienza University of Rome, Rome I-00185, Italy \\ ${ }^{3}$ Department of Physics, National Taiwan University, Taipei 10617, Taiwan \\ ${ }^{4}$ School of Physics Sciences, University of Chinese Academy of Sciences, Beijing 100039, China \\ ${ }^{5}$ Center for future High Energy Physics, Chinese Academy of Sciences, Beijing 100039, China \\ ${ }^{6}$ Centre for Cosmology, Particle Physics and Phenomenology (CP3), Université catholique de Louvain, \\ Chemin du Cyclotron, 2, B-1348 Louvain-la-Neuve, Belgium
}

(Received 29 October 2019; accepted 24 March 2020; published 16 April 2020)

\begin{abstract}
We study multi-Higgs final states in vector boson fusion (VBF) processes at the LHC and at future proton-proton colliders, focusing on the prospects for measurements at 27 and $100 \mathrm{TeV}$. We use an effective Lagrangian which includes higher-dimensional operators in the mass eigenstates which are relevant to VBF processes and relate this to specific parametrizations and models for new physics in the Higgs sector. We derive theoretical constraints on the parameter space from the unitarity of $2 \rightarrow n$ scattering amplitudes and apply the results to $V V \rightarrow h h$ and $h h h$ processes, where $V=W, Z$. As a result, we present constraints on differential distributions as appropriate to the study of $V V \rightarrow h h$ and $h h h$ processes.
\end{abstract}

DOI: 10.1103/PhysRevD.101.076012

\section{INTRODUCTION}

After the discovery of the Higgs boson with mass $m_{h} \approx$ $125 \mathrm{GeV}$ at the LHC [1,2], detailed measurements of all of its properties have become central to any search for new physics beyond the Standard Model (SM). In the SM, the Higgs boson has three types of interaction at tree level: (i) the Yukawa interaction with massive fermions, (ii) the interaction with electroweak gauge bosons ( $W^{ \pm}$and $Z$ ), and (iii) the cubic and quartic Higgs self-interactions. Establishing the last type of interaction is a crucial test of our current understanding of electroweak symmetry breaking. However, any direct measurement of Higgs selfinteractions involves producing two or more Higgs bosons in a single elementary process.

For all possible multi-Higgs production processes, the SM rates are very small. Beyond the SM, new effects may significantly enhance the rate, but any such enhancement is subject to generic relations from unitarity. In the current

\footnotetext{
*kilian@physik.uni-siegen.de

†sichunssun@gmail.com

*yanqishu@ucas.ac.cn

\$xiaoran.zhao@uclouvain.be

"zhao@physik.uni-siegen.de
}

Published by the American Physical Society under the terms of the Creative Commons Attribution 4.0 International license. Further distribution of this work must maintain attribution to the author(s) and the published article's title, journal citation, and DOI. Funded by SCOAP ${ }^{3}$. paper, we investigate multi-Higgs production in the context of vector-boson fusion topologies, compute production rates with appropriate cuts and selection criteria, and study the applicable unitarity constraints within an effectivetheory formalism. The results are intended to supply future studies of multi-Higgs production with generic limits of event distributions and rates that have to be considered in the physical interpretation of the analysis.

\section{A. Higgs-pair production in the SM}

The cubic Higgs self-coupling, hhh, contributes to processes that involve at least two Higgs bosons in the final state. At the LHC, the dominant process of Higgs-pair production in the $\mathrm{SM}$ is gluon-gluon fusion $(\mathrm{ggF})$ via a heavy top-quark loop. Current LHC data constrain the triple-Higgs self-coupling only very weakly [3]. Multiple groups have evaluated the potential for a first meaningful measurement of the triple-Higgs self-coupling at future high-luminosity runs of the LHC [4-10]. The considered decay channels of the Higgs pair include $W^{+} W^{-} W^{+} W^{-}$ [11,12], $b \bar{b} \gamma \gamma$ [13-18], $b \bar{b} W^{+} W^{-}$[19], $b \bar{b} \tau^{+} \tau^{-}$[20-22], $b \bar{b} \mu^{+} \mu^{-}$[13], $W^{+} W^{-} \gamma \gamma$ [23], and $b \bar{b} b \bar{b}[20,24,25]$. It is expected that the triple-Higgs self-coupling can be constrained within $40 \%$ accuracy after collecting $3 \mathrm{ab}^{-1}$ of data at the $14 \mathrm{TeV}$ LHC [26]. Beyond the LHC, at a future $100 \mathrm{TeV}$ hadron collider, the Higgs-pair production rate is enhanced significantly [9,15,27-32], allowing for a more accurate determination of the Higgs potential. 
Vector-boson fusion (VBF), $V V \rightarrow h h$, is a subdominant process of Higgs-pair production in hadron collisions [33]. The vector bosons $V=W^{ \pm}, Z$ are effectively radiated from incoming quarks. In addition to its dependence on the Higgs self-interaction, this process also depends on the $h V V$ and $h h V V$ couplings. The single-Higgs couplings $h V V$ can be determined from the precise measurement of the decay branching fractions $h \rightarrow W W^{*}$ and $h \rightarrow Z Z^{*}$ at the LHC, up to a common normalization factor. Current LHC data on the decay branching fractions for these channels are consistent with the SM predictions [34,35]. The Higgs-pair interaction $h h V V$ has not been accessible otherwise. In principle, the $V V \rightarrow h h$ process allows for a simultaneous extraction of this class of couplings and of the Higgs self-interaction $h h$.

The VBF mode of Higgs-pair production at hadron colliders has been studied in Refs. [36-40]. Beyond tree level, the NLO QCD correction enhances the cross section by $\sim 7 \%[41,42]$. In the high-luminosity mode of the LHC (HL-LHC) with $3 \mathrm{ab}^{-1}$ at $14 \mathrm{TeV}$, the $h h V V$ interaction can be constrained to $20 \%$ [38]. A $100 \mathrm{TeV}$ hadron collider has the potential to reduce the uncertainty down to $1 \%$ [39]. The $h h W W$ coupling is also accessible in the $W^{ \pm} W^{ \pm} h$ final state. In Ref. [43], it is found that this particular final state can constrain this coupling to $O(100 \%)$ at the HL-LHC and to $20 \%$ at a $100 \mathrm{TeV}$ collider.

There are further modes of Higgs-pair production in hadron collisions, namely, $t \bar{t} h h$ or $V h h$ production. The corresponding production rates are substantially smaller than in the $\mathrm{ggF}$ and VBF modes $[41,42,44-50]$.

\section{B. Triple-Higgs production in the SM}

The quartic Higgs coupling $h h h h$ is even more elusive, and experimentally establishing this interaction in the SM is a challenging task. Direct access requires processes with three Higgs bosons in the final state. In the dominant production channel at the LHC, $g g \rightarrow h h h$, the total cross section at $14 \mathrm{TeV}$ is only $O(0.01) \mathrm{fb}[51,52]$. As an alternative, the authors of Ref. [53] have considered triple Higgs-strahlung, $p p \rightarrow Z h h h$, but the cross section is also tiny. At a $100 \mathrm{TeV}$ hadron collider, triple-Higgs production via $\mathrm{ggF}$ can become observable in principle [54-60]. The cross section of $g g \rightarrow h h h$ at a $100 \mathrm{TeV}$ hadron collider is estimated to $5 \mathrm{fb}$ if NLO QCD corrections are included [54]. Various decay channels have been investigated in some detail, such as $h h h \rightarrow b \bar{b} b \bar{b} \gamma \gamma$ [55,56], hhh $\rightarrow$ $b \bar{b} b \bar{b} \tau \tau$ [57,60], and $h h h \rightarrow b \bar{b} W W^{*} W W^{*}$ [58]. These results are encouraging, but an unambiguous discovery of this process in the SM puts strong requirements on the performance of the detector and analysis.

Looking at the VBF mode of triple-Higgs production, $V V \rightarrow h h h$, the characteristics of the signal suggest a dedicated study despite the small expected event rate. The VBF topology, which implies forward jets with suppressed QCD activity in the central region, improves the signal-to-background ratio considerably. This process is sensitive to a $h h h V V$ interaction which does not exist in the SM but may be expected for a strongly interacting Higgs sector [61]. The amplitude also involves the lower-order $h V V$ and $h h V V$ couplings and is subject to gauge cancellations at high energy. For instance, an anomalous $h V V$ coupling would have a strong impact on triple-Higgs production in VBF [62].

At a future lepton collider, the situation is slightly more favorable. A high-luminosity $e^{+} e^{-}$collider in the energy range between $500 \mathrm{GeV}$ and $3 \mathrm{TeV}$ gives access to the Higgs-strahlung and VBF modes of Higgs-pair production and allows for a meaningful determination of the cubic Higgs self-interaction [63,64]. Furthermore, single-Higgs production processes enable an absolute determination of the $h V V$ couplings, which is an essential ingredient of an unambiguous determination of the Higgs potential in pairproduction processes. However, the SM cross section for triple-Higgs production in either $e^{+} e^{-} \rightarrow Z h h h$ or $e^{+} e^{-} \rightarrow$ $\nu \bar{\nu} h h$ does not rise above 1 ab $[65,66]$.

\section{Purpose and contents of this paper}

For a process that is as rare as triple-Higgs production, in the presence of large background, detection becomes a challenge even if an SM calculation predicts a sizable number of events. Fortunately, any disturbance of the SM interactions is likely to increase the expected event yield, possibly by a significant amount, so even a loose upper limit on the cross section should acquire physical meaning. To this end, it is important to know about model-independent upper limits, beyond which experimentally determined bounds would lose their immediate significance.

In this paper, we study double- and triple-Higgs production in VBF processes in an effective-field theory (EFT) approach. In this framework, anomalous effects are parametrized by the coefficients of higher-dimensional operators. By investigating the consequences of S-matrix unitarity for the amplitudes $V V \rightarrow h h$ and $V V \rightarrow h h h$, we constrain the energy-dependent parameter region where the EFT yields a valid parametrization. We use the packages WHIZARD [67] and Madgraph5_aMC@NLO [68] to compute the tree-level cross sections including all terms linear and bilinear in the EFT parameters. Evaluating numerical results at 14,27 , and $100 \mathrm{TeV}$, we turn the results into scale-dependent bounds on the model parameters. For a complete picture, it is important to treat Higgs-pair and triple-production processes on the same footing. By applying our methods to a detailed study of signal and background effects which we do not attempt here, it should become possible to properly gauge the achievable sensitivity of the collider to new-physics effects in the Higgs potential, in a largely model-independent fashion. 
The paper is organized as follows. In Sec. II, we establish the framework and introduce the generic effective Lagrangian, together with two more specific model scenarios where anomalous effects are present in the Higgs sector. In Sec. III, we derive unitarity relations that arise in general and in particular for this class of processes. To this end, we generalize the well-known formalism for quasielastic relativistic scattering processes to multiparticle final states and compute the specific constraints that arise within the EFT framework. In Sec. IV, we apply our findings to the phenomenology of Higgs-pair and triple-production processes, considering cut strategies, interference effects, and invariant-mass distributions. We conclude this paper with a discussion of our results in Sec. V.

\section{EFFECTIVE LAGRANGIAN FOR VBF HIGGS PRODUCTION}

For an unbiased approach to multi-Higgs production phenomenology and constraints, we would like to employ a framework that is as model independent as possible.
We choose to parametrize the Higgs interactions within an effective-theory framework. In practice, this may be considered as arising from the most general form-factor approach for the interactions of interest, where all form factors are expanded in powers of momentum. We keep the leading terms in the expansion. We do not introduce new fields, and we keep the infrared symmetries of the SM (QED, QCD) intact. The effective Lagrangian is expressed in terms of physical fields $W^{ \pm}, Z, h$, etc.

It is well known that such a framework can be rendered formally gauge invariant by introducing a nonlinear gauge representation for the electroweak interactions. Vice versa, the Lagrangian in terms of physical fields emerges from a generic nonlinear representation by selecting the unitary gauge, where Goldstone and ghost fields are eliminated. Furthermore, it is perturbatively equivalent to a standard effective-theory framework with linear gauge representation [69]. We have used the same framework for our previous study of multiple Higgs production in gluon-gluon fusion [58].

The effective Lagrangian can be written as follows:

$$
\begin{aligned}
\mathcal{L}_{\mathrm{EFT}}=\mathcal{L}_{\overline{\mathrm{SM}}}+\mathcal{L}_{h}+\mathcal{L}_{V V h}+\mathcal{L}_{V h}, \\
\mathcal{L}_{h}=-\lambda_{3} \frac{m_{h}^{2}}{2 v} h^{3}-\frac{\kappa_{5}}{2 v} h \partial^{\mu} h \partial_{\mu} h-\lambda_{4} \frac{m_{h}^{2}}{8 v^{2}} h^{4}-\frac{\kappa_{6}}{4 v^{2}} h^{2} \partial^{\mu} h \partial_{\mu} h+\cdots \\
\mathcal{L}_{V V h}=-\left(g_{W, b 1} \frac{h}{v}+g_{W, b 2} \frac{h^{2}}{2 v^{2}}+g_{W, b 3} \frac{h^{3}}{6 v^{3}}+\cdots\right) W_{\mu \nu}^{+} W^{-\mu \nu} \\
-\left(g_{A, b 1} \frac{h}{2 v}+g_{A, b 2} \frac{h^{2}}{4 v^{2}}+g_{A, b 3} \frac{h^{3}}{12 v^{3}}+\cdots\right) F_{\mu \nu} F^{\mu \nu} \\
-\left(g_{X, b 1} \frac{h}{v}+g_{X, b 2} \frac{h^{2}}{2 v^{2}}+g_{X, b 3} \frac{h^{3}}{6 v^{3}}+\cdots\right) F_{\mu \nu} Z^{\mu \nu} \\
-\left(g_{Z, b 1} \frac{h}{2 v}+g_{Z, b 2} \frac{h^{2}}{4 v^{2}}+g_{Z, b 3} \frac{h^{3}}{12 v^{2}}+\cdots\right) Z_{\mu \nu} Z^{\mu \nu} \\
\mathcal{L}_{V H}=g_{W, a 1} \frac{2 m_{W}^{2}}{v} h W^{+, \mu} W_{\mu}^{-}+g_{W, a 2} \frac{m_{W}^{2}}{v^{2}} h^{2} W^{\mu} W_{\mu}+g_{W, a 3} \frac{m_{W}^{2}}{3 v^{3}} h^{3} W^{\mu} W_{\mu} \\
+g_{Z, a 1} \frac{m_{Z}^{2}}{v} h Z^{\mu} Z_{\mu}+g_{Z, a 2} \frac{m_{Z}^{2}}{2 v^{2}} h^{2} Z^{\mu} Z_{\mu}+g_{Z, a 3} \frac{m_{Z}^{2}}{6 v^{3}} h^{3} Z^{\mu} Z_{\mu}+\cdots
\end{aligned}
$$

Dots indicate higher-dimensional interactions which are not relevant for the VBF Higgs production processes that we consider. We restrict the calculation to charge and parity symmetry (CP)-conserving interactions and therefore omit any $\mathrm{CP}$-violating operators.

In the SM at the tree level, we have the relations $\lambda_{3}=$ $\lambda_{4}=g_{W, a 1}=g_{W, a 2}=g_{Z, a 1}=g_{Z, a 2}=1$ and $\kappa_{5}=\kappa_{6}=g_{V}$, $b 1=g_{V, b 2}=g_{V, b 3}=g_{W, a 3}=g_{Z, a 3}=0$, where the subscript $V$ denotes $W, A, X, Z$. It is understood that the corresponding terms have been removed from $\mathcal{L}_{\overline{\mathrm{SM}}}$, such that they are not double counted.

The higher-order operators in the kinetic-energy term (proportional to $\kappa_{5}$ and $\kappa_{6}$ ) are redundant and can be eliminated by applying the equation of motion of the Higgs field or by a nonlinear transformation [70]. To eliminate $\kappa_{5}$, we may replace $h \rightarrow h+\frac{a}{2 v} h^{2}$ and get parameter shifts such as 
TABLE I. Parameters that contribute to the VBF Higgs production processes studied in this paper.

\begin{tabular}{lccc}
\hline \hline & $V V \rightarrow h$ & $V V \rightarrow h h$ & $V V \rightarrow h h h$ \\
\hline Parameters & $g_{V, a 1}, g_{V, b 1}$ & $g_{V, a 1}, g_{V, b 1}$ & $g_{V, a 1}, g_{V, b 1}$ \\
involved & $\ldots$ & $g_{V, a 2}, g_{V, b 2}, \lambda_{3}, \kappa_{5}$ & $g_{V, a 2}, g_{V, b 2}, \lambda_{3}, \kappa_{5}$ \\
& $\ldots$ & $\ldots$ & $g_{V, a 3}, g_{V, b 3}, \lambda_{4}, \kappa_{6}$ \\
\hline \hline
\end{tabular}

$$
\begin{aligned}
\lambda_{3} \rightarrow \lambda_{3}+a & \lambda_{4} \rightarrow \lambda_{4}+a^{2}+6 a \lambda_{3}, \\
\kappa_{5} \rightarrow \kappa_{5}-2 a & \kappa_{6} \rightarrow \kappa_{6}+5 a \kappa_{5}-2 a^{2}, \\
g_{W, a 2} \rightarrow g_{W, a 2}+a g_{W, a 1} & g_{W, a 3} \rightarrow g_{W, a 3}+3 a g_{W, a 2} .
\end{aligned}
$$

Choosing $a=\frac{1}{2} \kappa_{5}$ eliminates $\kappa_{5}$. Analogously, replacing $h \rightarrow h+\frac{b}{3 v^{2}} h^{3}$ results in

$$
\begin{gathered}
\lambda_{4} \rightarrow \lambda_{4}+4 b, \\
\kappa_{6} \rightarrow \kappa_{6}-4 b, \\
g_{W, a 3} \rightarrow g_{W, a 3}+2 b g_{W, a 1},
\end{gathered}
$$

so we can eliminate $\kappa_{6}$ with $b=\frac{\kappa_{6}}{4}$. To facilitate the comparison between parametrizations, we choose to retain these parameters in Table II below (cf. also Ref. [58]).

The phenomenological Lagrangian (2.1) provides a robust parametrization of new physics in the Higgselectroweak sector, under the condition that no new on shell states appear in the kinematically accessible range. In Table I, we summarize the dependency of various Higgs production processes on the coefficients in the effective Lagrangian, as can be read off from the contributing

\begin{tabular}{|c|c|c|}
\hline & SILH & Higgs inflation \\
\hline$\lambda_{3}$ & $\left(1+\frac{5}{2} c_{6} v^{2} / f^{2}\right)\left(1+\frac{3}{2} c_{6} v^{2} / f^{2}\right)^{-1} \zeta_{h}$ & $\left(1+6 \xi^{2} v^{2} / M_{p}^{2}\right)^{-1 / 2}$ \\
\hline$\lambda_{4}$ & $\left(1+\frac{15}{2} c_{6} v^{2} / f^{2}\right)\left(1+\frac{3}{2} c_{6} v^{2} / f^{2}\right)^{-1} \zeta_{h}^{2}$ & $\left(1+6 \xi^{2} v^{2} / M_{p}^{2}\right)^{-1}$ \\
\hline$\kappa_{5}$ & $-2 c_{H} v^{2} / f^{2} \zeta_{h}^{3}$ & $-12 v^{2} \xi^{2} / M_{p}^{2}\left(1+6 \xi^{2} v^{2} / M_{p}^{2}\right)^{-3 / 2}$ \\
\hline$\kappa_{6}$ & $-2 c_{H} v^{2} / f^{2} \zeta_{h}^{4}$ & $-12 v^{2} \xi^{2} / M_{p}^{2}\left(1+6 \xi^{2} v^{2} / M_{p}^{2}\right)^{-2}$ \\
\hline$g_{W, b 1}$ & $c_{H W} \frac{g^{2} v^{2}}{32 \pi^{2} f^{2}} \zeta_{h} \zeta_{W}^{2}$ & 0 \\
\hline$g_{W, b 2}$ & $c_{H W} \frac{g^{2} v^{2}}{32 \pi^{2} f^{2}} \zeta_{h}^{2} \zeta_{W}^{2}$ & 0 \\
\hline$g_{A, b 1}$ & $-c_{\gamma} \frac{g^{2} v^{2}}{8 \pi^{2} f^{2}} \frac{g^{2}}{g_{\rho}^{2}} \cos ^{2} \theta \zeta_{h} \zeta_{A}^{2}$ & 0 \\
\hline$g_{A, b 2}$ & $-c_{\gamma} \frac{g^{2} v^{2}}{8 \pi^{2} f^{2}} \frac{g^{2}}{g_{\rho}^{2}} \cos ^{2} \theta \zeta_{h}^{2} \zeta_{A}^{2}$ & 0 \\
\hline$g_{X, b 1}$ & $\begin{array}{c}\frac{g g^{\prime} v^{2}}{64 \pi^{2} f^{2}}\left[\left(c_{H W}-c_{H B}\right)+8 c_{\gamma} \frac{g^{2}}{g_{\rho}^{2}} \sin ^{2} \theta\right] \zeta_{h} \zeta_{A} \zeta_{Z} \\
+c_{\gamma} \frac{g^{2} v^{2}}{4 \pi^{2} f^{2}} \frac{g^{2}}{g_{\rho}^{2}} \cos ^{2} \theta \zeta_{h} \zeta_{A Z}^{2}\end{array}$ & 0 \\
\hline$g_{X, b 2}$ & $\begin{array}{c}\frac{g g^{\prime} v^{2}}{64 \pi^{2} f^{2}}\left[\left(c_{H W}-c_{H B}\right)+8 c_{\gamma} \frac{g^{2}}{g_{\rho}^{2}} \sin ^{2} \theta\right] \zeta_{h}^{2} \zeta_{A} \zeta_{Z} \\
+c_{\gamma} \frac{g^{2} v^{2}}{4 \pi^{2} f^{2}} \frac{g^{2}}{g_{\rho}^{2}} \cos ^{2} \theta \zeta_{h}^{2} \zeta_{A Z}^{2}\end{array}$ & 0 \\
\hline$g_{Z, b 1}$ & $\begin{array}{c}\frac{g^{2} v^{2}}{32 \pi^{2} f^{2}}\left(c_{H W}+c_{H B} \tan ^{2} \theta\right) \zeta_{h} \zeta_{Z}^{2}-c_{\gamma} \frac{g^{2} v^{2}}{8 \pi^{2} f^{2}} \frac{g^{2}}{\frac{2}{\rho}} \cos ^{2} \theta \zeta_{h} \zeta_{A Z}^{2} \\
-\frac{g g^{\prime} v^{2}}{64 \pi^{2} f^{2}}\left[\left(c_{H W}-c_{H B}\right)+8 c_{\gamma} \frac{g^{2}}{g_{\rho}^{2}} \sin ^{2} \theta\right] \zeta_{h} \zeta_{A Z} \zeta_{Z}\end{array}$ & 0 \\
\hline$g_{Z, b 2}$ & $\begin{array}{c}\frac{g^{2} v^{2}}{32 \pi^{2} f^{2}}\left(c_{H W}+c_{H B} \tan ^{2} \theta\right) \zeta_{h}^{2} \zeta_{Z}^{2}-c_{\gamma} \frac{g^{2} v^{2}}{8 \pi^{2} f^{2}} \frac{g^{2}}{g_{\rho}^{2}} \cos ^{2} \theta \zeta_{h}^{2} \zeta_{A Z}^{2} \\
-\frac{g g^{\prime} v^{2}}{64 \pi^{2} f^{2}}\left[\left(c_{H W}-c_{H B}\right)+8 c_{\gamma} \frac{g^{2}}{g_{\rho}^{2}} \sin ^{2} \theta\right] \zeta_{h} \zeta_{A Z} \zeta_{Z}\end{array}$ & 0 \\
\hline$g_{W, a 1}$ & {$\left[1-\left(c_{W} \frac{g^{2} v^{2}}{m_{\rho}^{2}}+c_{H W} \frac{g^{2} v^{2}}{16 \pi^{2} f^{2}}\right)\right] \zeta_{h} \zeta_{W}^{2}$} & $\left(1+6 \xi^{2} v^{2} / M_{p}^{2}\right)^{-1 / 2}$ \\
\hline$g_{Z, a 1}$ & {$\left[1-\left(c_{W} \frac{g^{2} v^{2}}{m_{\rho}^{2}}+c_{B} \frac{g^{2} v^{2}}{m_{\rho}^{2}}+c_{H W} \frac{g^{2} v^{2}}{16 \pi^{2} f^{2}}+c_{H B} \frac{g^{2} v^{2}}{16 \pi^{2} f^{2}}\right)\right] \zeta_{h} \zeta_{Z}^{2}$} & $\left(1+6 \xi^{2} v^{2} / M_{p}^{2}\right)^{-1 / 2}$ \\
\hline$g_{W, a 2}$ & {$\left[1-3\left(c_{W} \frac{g^{2} v^{2}}{m_{\rho}^{2}}+c_{H W} \frac{g^{2} v^{2}}{16 \pi^{2} f^{2}}\right)\right] \zeta_{h}^{2} \zeta_{W}^{2}$} & $\left(1+6 \xi^{2} v^{2} / M_{p}^{2}\right)^{-1}$ \\
\hline$g_{Z, a 2}$ & {$\left[1-3\left(c_{W} \frac{g^{2} v^{2}}{m_{\rho}^{2}}+c_{B} \frac{g^{g^{2} v^{2}}}{m_{\rho}^{2}}+c_{H W} \frac{g^{2} v^{2}}{16 \pi^{2} f^{2}}+c_{H B} \frac{g^{2} v^{2}}{16 \pi^{2} f^{2}}\right)\right] \zeta_{h}^{2} \zeta_{Z}^{2}$} & $\left(1+6 \xi^{2} v^{2} / M_{p}^{2}\right)^{-1}$ \\
\hline$g_{W, a 3}$ & From dim-8 operators or higher & $\mathcal{O}(\xi)$ \\
\hline$g_{Z, a 3}$ & From dim-8 operators or higher & $\mathcal{O}(\xi)$ \\
\hline
\end{tabular}
Feynman diagrams.

TABLE II. Relations between the phenomenological Lagrangian parameters in (2.1)-(2.4) (first column), the SILH effective Lagrangian A 1 (second column), and the free parameters of the Higgs-inflation model, Sec. A 1. Note the extra parameters $\zeta_{h}^{n}, \zeta_{W}^{n}, \zeta_{Z}^{n}, \zeta_{A}^{n}, \zeta_{A Z}^{n}$ (defined in Eq. (A34)-(A41) and the $\left(1+6 \xi^{2} v^{2} / M_{p}^{2}\right)^{-1 / 2}$ factor, induced by the Higgs and gauge-boson wave-function normalization, respectively. 
Table II contains the actual translation between parameter sets. We consider two actual models: the strongly interacting light Higgs (SILH) model and Higgs-inflation model. Details of deriving the relation between the SILH Lagrangian and the effective Lagrangian (2.1), the relation between the Higgs-inflation model and the effective Lagrangian, including conventions, are given in A 2.

In Table II, we list the coefficient expressions for the Higgs-inflation model and relate them to the SILH operator basis and to the Higgs Lagrangian that we use for our study. It is evident that the SILH operator basis, which is appropriate for a generic strongly interacting model, incorporates directions in parameter space which are absent in the more specific model of Higgs inflation. Dedicated measurements of Higgs self-interactions become essential if such a class of model is realized.

\section{CONSTRAINTS ON PARAMETERS FROM THE UNITARITY OF S MATRIX}

When adopting an EFT for calculations, we accept a truncated Taylor expansion as a model for any scattering amplitude, distribution or cross section. A term of dimension $d$ in the Lagrangian generates uncanceled factors that rise proportional to $E^{d-4}$ in the amplitude, where $E$ is the overall energy scale in a scattering process [71]. Due to this growth, the model ceases to be weakly interacting at some scale.

The scale where the scattering strength has to saturate, can be deduced from the optical theorem, i.e., unitarity. Physically, the theorem describes the effect of rescattering which occurs whenever overlapping field amplitudes become sufficiently large. In this work, we want to turn the argument around and determine bounds for EFT parameters such that sizable rescattering corrections do not occur below some arbitrary scale $Q$, for the concrete processes of multi-Higgs production in VBF.

In the simplest case of spinless $2 \rightarrow 2$ scattering, exploiting the optical theorem is textbook knowledge. A partial-wave decomposition will reformulate the restricted $\mathcal{S}$ operator as a discrete matrix which can be diagonalized for convenience (cf., e.g., Ref. [72]).

The extension to relativistic $2 \rightarrow n$ scattering $(n>2)$, in the context of the SM and its extensions, is not as familiar. There is no obvious decomposition of the final state. Apparently, we have to consider $m \rightarrow n$ contributions to the scattering operator which do not correspond to physical scattering processes, and reformulating the problem into matrix equations is no longer straightforward. To arrive at useful inequalities, previous work introduced extra assumptions [73-75]. Neglecting spin also simplifies the problem considerably [76].

Below, we adopt a generic approach, which we then apply to the particular case at hand. It turns out that the exact form of decomposition does not matter for the purpose of deriving phenomenologically viable parameter bounds. In the particular case of EFT operators, complications from kinematical dependencies are largely absent.

\section{A. General unitarity constraints}

Unitarity is the conservation of probability in a quantum theory, applied to the $\mathcal{S}$ operator that encodes the scattering of observable particles $\mathcal{S}^{\dagger} \mathcal{S}=1$. Its nontrivial part $\mathcal{T}$, defined by $\mathcal{S}=1+i \mathcal{T}$, satisfies the universal relation

$$
-i\left(\mathcal{T}-\mathcal{T}^{\dagger}\right)=\mathcal{T}^{\dagger} \mathcal{T} \text {. }
$$

We are interested in unitarity conditions for matrix elements between asymptotic states which consist of a finite number $n_{a}$ of particles with well-defined masses. We denote multiparticle states collectively by $\left|\alpha, \Phi_{a}\right\rangle$, where $\Phi_{a}$ is a shorthand for the kinematical configuration of $n_{a}$ on shell four-momenta (the phase-space point), and $\alpha$ denotes the set of discrete quantum numbers such as helicity and color. Furthermore, we fix the total momentum of a multiparticle state $a$ to $p_{a}$. With this constraint, the manifold of configurations $\left(\alpha, \Phi_{a}\right)$ becomes a compact manifold for each fixed $n_{a}$.

Momentum conservation allows us to introduce the matrix elements of the scattering amplitude operator $\mathcal{M}$ between the initial state $\left|\alpha, \Phi_{a}\right\rangle$ and the final state $\left|\beta, \Phi_{b}\right\rangle$,

$$
\left\langle\beta, \Phi_{b}|\mathcal{T}| \alpha, \Phi_{a}\right\rangle=(2 \pi)^{4} \delta^{(4)}\left(p_{a}-p_{b}\right)\left\langle\beta, \Phi_{b}|\mathcal{M}| \alpha, \Phi_{a}\right\rangle .
$$

We take matrix elements on both sides of Eq. (3.1) and insert a complete set of multiparticle states $\left|\gamma, \Phi_{c}\right\rangle$,

$$
\begin{aligned}
- & i\left[\left\langle\beta, \Phi_{b}|\mathcal{M}| \alpha, \Phi_{a}\right\rangle-\left\langle\alpha, \Phi_{a}|\mathcal{M}| \beta, \Phi_{b}\right\rangle^{*}\right] \\
& =\sum_{\gamma} \int \mathrm{d} \Phi_{c}\left\langle\gamma, \Phi_{c}|\mathcal{M}| \beta, \Phi_{b}\right\rangle^{*}\left\langle\gamma, \Phi_{c}|\mathcal{M}| \alpha, \Phi_{a}\right\rangle,
\end{aligned}
$$

where $\mathrm{d} \Phi_{c}$ denotes the canonical Lorentz-invariant measure on the phase space $\left\{\Phi_{c}\right\}$ constrained by $p_{c}=p_{a}=p_{b}$.

For calculations, we may introduce a bijective mapping between the unit hypercube in $d_{a}=3 n_{a}-4$ dimensions, $\left\{x_{a} \in \mathbb{R}^{d_{a}} ; 0<\left(x_{a}\right)_{i}<1\right\}$ and the manifold $\left\{\Phi_{a}\right\}$, for each fixed $n_{a}$. For instance, we may factorize phase space as a tree consisting of $n_{a}-1$ momentum splittings of type $1 \rightarrow 2$, with $p_{a}$ at the root. There are $2\left(n_{a}-1\right)$ angular variables and $n_{a}-2$ invariant-mass variables. This mapping introduces a Jacobian $J_{a}\left(x_{a}\right)=\mathrm{d} \Phi_{a} / \mathrm{d} x_{a}$, which should incorporate symmetry factors where appropriate. The construction provides a method of evaluating phasespace integrals that has become standard and preserves overall Lorentz invariance. If we introduce amplitude functions which include the Jacobian factors as follows:

$M_{\beta \alpha}\left(x_{b}, x_{a}\right)=J_{b}^{1 / 2}\left(x_{b}\right)\left\langle\beta, \Phi_{b}\left(x_{b}\right)|\mathcal{M}| \alpha, \Phi_{a}\left(x_{a}\right)\right\rangle J_{a}^{1 / 2}\left(x_{a}\right)$,

Eq. (3.1) takes the form 


$$
\begin{aligned}
-i & {\left[M^{\beta \alpha *}\left(x_{b}, x_{a}\right)-M^{\alpha \beta}\left(x_{a}, x_{b}\right)\right] } \\
& =\sum_{\gamma} \int \mathrm{d} x_{c} M^{\gamma \beta *}\left(x_{c}, x_{b}\right) M^{\gamma \alpha}\left(x_{c}, x_{a}\right) .
\end{aligned}
$$

If massless particles are involved, the sum over intermediate states is infinite, and the matrix elements contain nonintegrable infrared, collinear, and Coulomb singularities, so the integrals do not converge. To remedy this, we may introduce some version of phase-space slicing and sum over nearly degenerate states, which introduces indefinite particle numbers $[77,78]$. However, in the present context where we are studying the production of massive bosons, we focus on anomalous couplings of the Higgs boson. As we do not change the couplings of massless particles, we may ignore this complication, and assume that all external and internal states are massive. The sum over intermediate states then is a finite sum, the matrix elements and the Jacobians are finite, and the integration manifold [the union of the unit hypercubes for all contributing $\left.\left(n_{a}, \alpha\right)\right]$ is compact.

In such a situation, it is possible to introduce a scalar product of square-integrable functions on the integration manifold and to find a complete basis of functions which are mutually orthonormal with respect to this scalar product. For instance, choosing the canonical scalar product, we could take a straightforward Fourier expansion. A more physical choice could involve spherical harmonics for the normalized angular variables and an arbitrary basis for the invariant-mass variables. In the two-particle case where there are no free invariant masses, this becomes the standard partial-wave expansion. We note that for each particle combination $a$, we may choose a different kind of expansion for the corresponding phase space $\Phi_{a}\left(x_{a}\right)$.

We adopt, for simplicity, the canonical scalar product and a corresponding orthonormal basis $\left\{H_{A}^{\alpha}\left(x_{a}\right)\right\}$ on each $\alpha$ phase space,

$$
\int \mathrm{d} x_{a} H_{A}^{\alpha *}\left(x_{a}\right) H_{B}^{\alpha}\left(x_{a}\right)=\delta_{A B},
$$

where $A$ is an appropriate (multi-)index which labels the basis functions. We expand the amplitudes as

$$
M^{\beta \alpha}\left(x_{b}, x_{a}\right)=2 \sum_{A B} a_{A B}^{\alpha \beta} H_{A}^{\alpha}\left(x_{a}\right) H_{B}^{\beta *}\left(x_{b}\right)
$$

and thus express the scattering in terms of an actual matrix with elements $a_{A B}^{\alpha \beta}{ }^{1}$ Explicitly, the coefficients are

$$
a_{A B}^{\alpha \beta}=\frac{1}{2} \int \mathrm{d} x_{a} \mathrm{~d} x_{b} H_{A}^{\alpha *}\left(x_{a}\right) H_{B}^{\beta}\left(x_{b}\right) M^{\beta \alpha}\left(x_{b}, x_{a}\right) .
$$

They take complex values and depend only on the total momentum, $a_{A B}^{\alpha \beta}=a_{A B}^{\alpha \beta}\left(p_{a}\right)$, where $p_{a}=p_{b}$. If we choose

\footnotetext{
${ }^{1}$ The factor 2 has been inserted for consistency with the standard two-particle partial-wave expansion.
}

a phase-space parametrization which preserves Lorentz invariance, the coefficients depend only on $s=p_{a}^{2}$.

We obtain a discrete version of Eq. (3.5) [79,80],

$$
-i\left(a_{A B}^{\alpha \beta}-a_{B A}^{\beta \alpha *}\right)=2 \sum_{\gamma} \sum_{C} a_{A C}^{\alpha \gamma} a_{B C}^{\beta \gamma *},
$$

where all coefficients are finite and the sums are convergent if the simplifications regarding massless states are applied, as described above.

Equation (3.9) encodes all unitarity relations of the scattering matrix in question. To derive constraints on individual amplitudes, we need a positivity condition. We may diagonalize the scattering matrix and obtain exact relations for superpositions of states. Alternatively, we may derive less comprehensive but phenomenologically more useful relations by focusing on diagonal matrix elements, i.e., $\alpha=\beta$ and $A=B$,

$$
\begin{array}{r}
-i\left(a_{A A}^{\alpha \alpha}-a_{A A}^{\alpha \alpha *}\right)=2 \sum_{\gamma} \sum_{C}\left|a_{A C}^{\alpha \gamma}\right|^{2}, \\
=2\left|a_{A A}^{\alpha \alpha}\right|^{2}+2 \sum_{C \neq A}\left|a_{A C}^{\alpha \alpha}\right|^{2}+2 \sum_{\gamma \neq \alpha} \sum_{C}\left|a_{A C}^{\alpha \gamma}\right|^{2} .
\end{array}
$$

To cast this in the intuitive geometry of the Argand circle, we may express the diagonal amplitude in terms of its real and imaginary parts and write

$\left|\operatorname{Re} a_{A A}^{\alpha \alpha}\right|^{2}+\left|\operatorname{Im} a_{A A}^{\alpha \alpha}-\frac{1}{2}\right|^{2}+\sum_{C \neq A}\left|a_{A C}^{\alpha \alpha}\right|^{2}+\sum_{\gamma \neq \alpha} \sum_{C}\left|a_{A C}^{\alpha \gamma}\right|^{2}=\frac{1}{4}$.

That is, each complex-valued elastic amplitude $a_{A A}^{\alpha \alpha}(s)$ must lie on a circle with radius $r$ around $i / 2$, where the elastic radius $r=1 / 2$ is reduced by the total contribution of all inelastic channels.

The exact relation (3.12) yields strict upper bounds for the elastic amplitude as well as for the total inelastic contribution, which trivially translates into a bound for each individual final state in this representation. We read off

$$
\begin{aligned}
\left|\operatorname{Re} a_{A A}^{\alpha \alpha}\right|^{2} & \leq \frac{1}{4} \\
\left|\operatorname{Im} a_{A A}^{\alpha \alpha}-\frac{1}{2}\right|^{2} & \leq \frac{1}{4} \\
\sum_{C \neq A}\left|a_{A C}^{\alpha \alpha}\right|^{2} & \leq \frac{1}{4} \\
\sum_{\gamma \neq \alpha} \sum_{C}\left|a_{A C}^{\alpha \gamma}\right|^{2} & \leq \frac{1}{4} .
\end{aligned}
$$

Examples for the application of these bounds, referring also to the treatments in Refs. [72-74,76], can be found in Appendix B. 
(a)

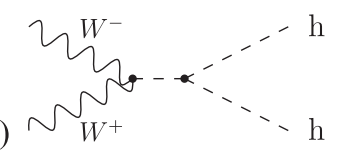

(b)

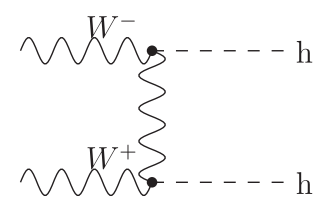

(c)

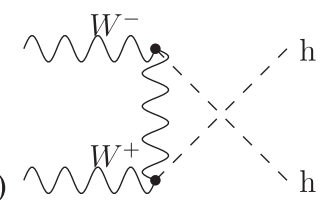

(d)

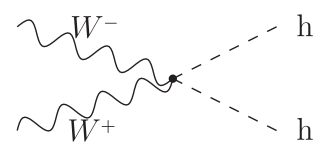

FIG. 1. Four types of Feynman diagrams which contribute to the processes $W^{+} W^{-} \rightarrow h h$.

The last inequality in Eq. (3.13) gives the unitarity constraints on inelastic scattering. The important observation is that it is independent of the chosen basis in multiparticle phase space, $\left\{H^{\gamma}\right\}$. To see this, we define the coefficients $b_{A}^{\alpha \gamma}$ as follows:

$$
\begin{aligned}
b_{A}^{\alpha \gamma} \equiv & \frac{1}{4} \int \mathrm{d} x_{a} \mathrm{~d} x_{b} \mathrm{~d} x_{c} H_{A}^{\alpha *}\left(x_{a}\right) H_{A}^{\alpha}\left(x_{b}\right) \\
& \times M^{\gamma \alpha *}\left(x_{c}, x_{b}\right) M^{\gamma \alpha}\left(x_{c}, x_{a}\right),
\end{aligned}
$$

which is clearly independent of $H^{\gamma}$. Using the expansion in Eq. (3.7), we find

$$
b_{A}^{\alpha \gamma}=\sum_{C}\left|a_{A C}^{\alpha \gamma}\right|^{2} \geq 0 .
$$

The unitarity constraint for inelastic scattering can be written as

$$
\sum_{\gamma \neq \alpha} b_{A}^{\alpha \gamma} \leq \frac{1}{4}
$$

The problem has been reformulated as a set of discrete matrix equations. Diagonalization becomes a possibility also for the generic case, although analytical solutions do not exist for realistic applications [81].

We are interested in inelastic scattering processes, $V V \rightarrow$ $h h$ and $V V \rightarrow h h h$. For those, the inequality (3.16) constrains the sum over all contributions to a particular final state. Moreover, we read off the weaker constraint

$$
0 \leq b_{A}^{\alpha \gamma} \leq \frac{1}{4}
$$

for each individual contribution $\gamma$. In fact, the offending terms in the EFT turn out to either not depend on the phasespace point at all, or otherwise the dependence is well controlled, so that meaningful bounds can easily be computed. We arrive at a set of conservative bounds on the parameter space even without an analytical solution. A more detailed calculation could further narrow down the parameter space but not widen it.

\section{B. Unitarity constraints arising from $V V \rightarrow h h$}

We now apply the generic formalism to the two-particle inelastic scattering process $V V \rightarrow h h$. In the following, we derive the formulas for $W^{+} W^{-} \rightarrow h h$. Analogous results for $Z Z \rightarrow h h$ can be obtained by replacing the couplings accordingly and taking into account the symmetry factor from two identical $Z$ boson. We assume that the on shell approximation is justified for the purpose of deriving unitarity bounds, i.e., we treat the incoming vector bosons as on shell with a pair invariant mass $m\left(W^{+} W^{-}\right)=$ $m(h h)=\hat{s}$. In the actual process, the incoming propagators are spacelike with a virtuality of $O\left(m_{W}^{2}\right)$; cf. Ref. [82] for the possibility of relaxing that assumption.

We are looking at an inelastic channel. If we expand in an orthonormal basis as described in the preceding subsection, we obtain

$$
b_{A}^{W^{+} W^{-} \rightarrow h h}(\hat{s}) \equiv \sum_{C}\left|a_{A C}^{W^{+} W^{-} \rightarrow h h}(\hat{s})\right|^{2} \leq \frac{1}{4},
$$

where $A$ and $C$ are (multi-)indices for the initial-state and final-state basis, respectively. We note that the initial-state particles carry spin as well as momentum, while the finalstate phase space manifold is trivially given by the unit sphere, for fixed energy $\sqrt{\hat{s}}$.

As shown in Fig. 1, there are four distinct Feynman diagrams which contribute to the $W^{+} W^{-} \rightarrow h h$ process in the SM, and this breakdown remains valid in the EFT,

$$
M\left(W^{+} W^{-} \rightarrow h h\right)=M_{s}+M_{t}+M_{u}+M_{4} .
$$

We refer to these as the $s$ channel, $t$ channel, $u$ channel, and contact-interaction amplitudes, respectively.

In the high-energy limit $s \gg m_{W}^{2}, m_{H}^{2}$, the leading contribution in the EFT is proportional to $s$. We thus write a series expansion as follows, in terms of the rescaled energy $\sqrt{s / v^{2}}$ as a dimensionless expansion parameter,

$$
M\left(W^{+} W^{-} \rightarrow h h\right)=\sum_{i=0}^{+\infty} m_{i}\left(\frac{\sqrt{s}}{v}\right)^{2-i},
$$

where $m_{i}$ are the coefficients in the expansion. In Table III, we list the prefactors of the leading contribution for each

TABLE III. Leading contribution $m_{0}$ to the helicity amplitudes for $V V \rightarrow h h$, broken down by type of Feynman diagram. The notation $\mathcal{O}\left(g_{W, b 2}^{2}\right)$ indicates that the contribution is nonzero but depends on the phase-space point, proportional to the coupling constants $g_{W, b 2}^{2}$.

\begin{tabular}{lccc}
\hline \hline Helicity configuration & ++ & +- & 00 \\
\hline$s$ channel & $\frac{1}{2} \kappa_{5} g_{W, b 1}$ & 0 & $\frac{1}{2} \kappa_{5} g_{W, a 1}$ \\
$t, u$ channel & $2 g_{W, b 1}^{2}$ & $\mathcal{O}\left(g_{W, b 1}^{2}\right)$ & $-g_{W, a 1}^{2}$ \\
Contact interaction & $g_{W, b 2}$ & 0 & $g_{W, a 2}$ \\
\hline \hline
\end{tabular}




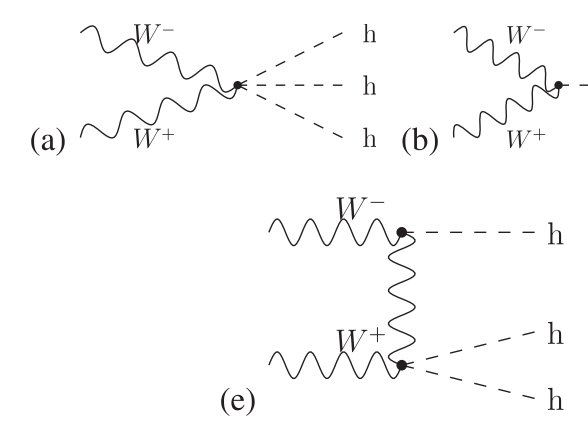

(e)

FIG. 2. Seven types of Feynman diagrams which contribute to the processes $W^{+} W^{-} \rightarrow h h h$. amplitude and each one of the four independent helicity modes. The amplitudes of all other helicity modes are related to the four modes that we include in the table. We find that with the exception of one term $(t / u$-channel +-$)$, all leading contributions are independent of the kinematics. These table entries translate directly into bounds for amplitude coefficients. We also observe that only the ,+++- , and 00 modes lead to amplitudes rising proportional to $s$, so we may focus on those when considering unitarity bounds.

Angular-momentum conservation directs the choice of a convenient phase-space basis for the initial state of two vector bosons. We couple helicity with orbital angular momentum to total angular momentum $j$, i.e., adopt the Wigner D-matrix formalism (cf. Appendix B) in analogy with the formalism developed for quasielastic processes [83].

We thus derive individual bounds for amplitude coefficients $b_{j}\left(h_{1} h_{2}\right)$,

$$
b_{j}\left(h_{1} h_{2}\right) \leq \frac{1}{4}, \quad \text { where } h_{i}=+-0 .
$$

The strongest bounds on the EFT coefficients that we obtain for this process are the following ones:

$$
\begin{gathered}
b_{0}(00)=\frac{s^{2}}{2^{9} \pi^{2} v^{4}}\left|g_{W, a 2}-g_{W, a 1}^{2}+\frac{1}{2} \kappa_{5} g_{W, a 1}\right|^{2} \leq \frac{1}{4}, \\
b_{0}(++)=\frac{s^{2}}{2^{9} \pi^{2} v^{4}}\left|g_{W, b 2}+2 g_{W, b 1}^{2}+\frac{1}{2} \kappa_{5} g_{W, b 1}\right|^{2} \leq \frac{1}{4}, \\
b_{2}(+-)=\frac{s^{2}}{3 \times 2^{10} \pi^{2} v^{4}} g_{W, b 1}^{4} \leq \frac{1}{4} .
\end{gathered}
$$

In particular, the +- mode contributes a bound on $g_{W, b 1}$, i.e., the $h W_{T}^{+} W_{T}^{-}$interaction, which is independent of the other EFT parameters.

We have deliberately neglected the constraint from the +0 helicity amplitude. The amplitude should respect the following constraint:

$$
b_{2}(+0)=\frac{s m_{W}^{2}}{32^{7} \pi^{2} v^{4}} g_{W, a 1}^{2} g_{W, b 1}^{2} \leq \frac{1}{4} .
$$

In principle, we can also derive a bound of $g_{W, b 1}$ from this equation also, instead from Eq. (3.24). But it is noticed that only when $g_{W, a 1} \geq \sqrt{\sqrt{3}} \pi / m_{W} \approx 7$, the new bound from $b_{2}(+0)$ could be more stringent than that from $b_{2}(+-)$. As it is known that a large deviation of $g_{W, a 1}=1$ is ruled out by the Higgs decay data from current LHC measurements; therefore, we will neglect the bound derived from $b_{2}(+0)$ here and after.

\section{Unitarity constraints arising from $V \boldsymbol{V} \rightarrow \boldsymbol{h} h \boldsymbol{h}$}

The helicity amplitudes of the process $W^{+} W^{-} \rightarrow h h h$ are associated with seven distinct types of Feynman diagrams, Fig. 2. Similar to $W^{+} W^{-} \rightarrow h h$, in the highenergy limit, the amplitude can be expanded as a series in powers of $\sqrt{s / v^{2}}$,

$$
M\left(W^{+} W^{-} \rightarrow h h h\right)=\sum_{i=0}^{+\infty} m_{i} v^{-1}\left(\frac{\sqrt{s}}{v}\right)^{2-i} .
$$

We list the leading term $m_{0}$ in Table IV for each helicity combination. Wherever the coefficient is phase-space dependent, we denote it as $\mathcal{O}(C)$, where $C$ is a combination of coupling constants.

Since this is also an inelastic channel, we obtain unitarity bounds on the $b$-coefficients defined in Eq. (3.14),

TABLE IV. Leading contribution $m_{0}$ to the helicity amplitudes in the high-energy limit for $V V \rightarrow h h h$, broken down by type of Feynman diagram.

\begin{tabular}{cccc}
\hline \hline & ++ & +- & 00 \\
\hline $\mathrm{a}$ & $g_{W, b 3}$ & 0 & $g_{W, a 3}$ \\
$\mathrm{~b}$ & $\frac{1}{2} g_{W, b 1} \kappa_{6}$ & 0 & $\frac{1}{2} g_{W, a 1} \kappa_{6}$ \\
$\mathrm{c}$ & $\frac{3}{2} g_{W, b 2} \kappa_{5}$ & 0 & $\frac{3}{2} g_{W, a 2} \kappa_{5}$ \\
$\mathrm{~d}$ & $g_{W, b 1} \kappa_{5}^{2}$ & 0 & $g_{W, a 1} \kappa_{5}^{2}$ \\
$\mathrm{e}$ & $6 g_{W, b 1} g_{W, b 2}$ & $\mathcal{O}\left(g_{W, b 1} g_{W, b 2}\right)$ & $-4 g_{W, a 1} g_{W, a 2}$ \\
$\mathrm{f}$ & $\mathcal{O}\left(g_{W, b 1}^{3}\right)$ & $\mathcal{O}\left(g_{W, b 1}^{3}\right)$ & $4 g_{W, a 1}^{3}$ \\
$\mathrm{~g}$ & $3 g_{W, b 1}^{2} \kappa_{5}$ & $\mathcal{O}\left(g_{W, b 1}^{2} \kappa_{5}\right)$ & $-2 g_{W, a 1}^{2} \kappa_{5}$ \\
\hline \hline
\end{tabular}




$$
b_{A}^{W^{+} W^{-} \rightarrow h h h}(\hat{s}) \leq \frac{1}{4}
$$

Note that the $b$ coefficients are independent of the phasespace parametrization and of the basis functions for the triple-Higgs system; only the phase-space parametrization and the basis functions for the $W$-boson pair do matter. As discussed in Appendix B, after we choose the Wigner D matrix as our basis for the $W^{+} W^{-}$state, the $b$ coefficients are diagonal and can be denoted as $b_{j}\left(h_{1} h_{2}\right)$, where $j$ represents the total angular momentum, and $h_{i}=+,-, 0$ are the helicities of the two $W$ bosons. We calculate the (reduced) $b$-coefficients directly according to Eq. (3.14). Although the result is independent of the phase-space parametrization for the triple-Higgs system, an explicit expression is required for phase-space integration; we adopt the form given in Appendix B 4. We give the results for the three helicity modes as follows:
(1) For the 00 helicity mode, the amplitude is constant in phase space. The corresponding bound becomes

$$
\begin{aligned}
b_{0}(00)= & \frac{s^{3}}{3 \times 2^{14} \pi^{4} v^{6}} \mid g_{W, a 3} \\
& +\frac{1}{2} g_{W, a 1} \kappa_{6}+\frac{3}{2} g_{W, a 2} \kappa_{5}+g_{W, 1} \kappa_{5}^{2} \\
& -4 g_{W, a 1} g_{W, a 2}+4 g_{W, a 1}^{3}-\left.2 g_{W, a 1}^{2} \kappa_{5}\right|^{2} \leq \frac{1}{4} .
\end{aligned}
$$

(2) For the ++ helicity mode, the type-f contribution is phase-space dependent, and it yields a nonzero $b_{j}$ for $j>0$. However, we checked that the dependence is of minor importance, and the bounds from $b_{j} \leq \frac{1}{4}$ with $j>0$ turn out to be much weaker than the bounds from $W^{+} W^{-} \rightarrow h h$. Therefore, we only quote $b_{0}$ here,

$$
\begin{aligned}
b_{0}(++)= & \frac{s^{3}}{3 \times 2^{14} \pi^{4} v^{6}}\left(\mid g_{W, b 3}+\frac{1}{2} g_{W, b 1} \kappa_{6}+\frac{3}{2} g_{W, b 2} \kappa_{5}+g_{W, b 1} \kappa_{5}^{2}\right. \\
& \left.+6 g_{W, b 1} g_{W, b 2}+f_{1} g_{W, b 1}^{3}-\left.3 g_{W, b 1}^{2} \kappa_{5}\right|^{2}+f_{2} g_{W, b 1}^{6}\right) \leq \frac{1}{4},
\end{aligned}
$$

with $f_{1}=7.49994 \pm 0.00005$ and $f_{2}=0.0658 \pm 0.0006$ computed by numerical integration. The negligible $f_{2}$ reflects the fact that the dependence of $g_{W, b 1}^{3}$ on phase space is small.

(3) For the +- helicity mode, only $b_{j}$ with $j=2,4, \ldots$ are nonzero, and among them the largest one is $b_{2}$, which is given by

$$
b_{2}(+-)=\frac{s^{3}}{3 \times 2^{14} \sqrt{6} \pi^{4} v^{6}}\left|g_{W, b 1} g_{W, b 2}+2 g_{W, b 1}^{3}+\frac{1}{2} g_{W, b 1}^{2} \kappa_{5}\right|^{2} \leq \frac{1}{4} .
$$

Similar to the $V V \rightarrow h h$ case, we find that the constraint from the $b_{2}(+0)$ helicity mode amplitude only contributes to $m_{1}$, which belongs to a subleading one. It is noticed that the related amplitude depends on the values of $g_{W, a 1}, g_{W, a 2}$, and $\kappa_{5}$. A meaningful constraint derived from $b_{2}(+0)$ mode could only be more stringent when the values of these parameters could have very large deviation from the prediction of the SM. Although such a possibility might not be forbidden, we will not consider it in our following discussion.

\section{MULTI-HIGGS PRODUCTION IN VBF PROCESSES IN THE EFT APPROACH}

In this section, we consider the phenomenology of multi-Higgs production in VBF together with basic strategies to isolate the signals at a high-energy hadron collider. The impact of anomalous couplings, introduced via the EFT Lagrangian, depends on the final-state kinematics, and has to be understood in order to evaluate the physical implications of assigning unitarity bounds to the EFT parameter set.

We have computed the cross sections for the processes $p p \rightarrow h h j j$ and $p p \rightarrow h h h j j$ including the full dependence on the higher-dimensional operator coefficients, represented by the free parameters of the phenomenological Lagrangian (2.1). To enhance the contribution of the VBF subprocess, we apply standard VBF cuts, as listed in Table V. We display results for the $14 \mathrm{TeV} \mathrm{LHC}$, for a

TABLE V. Acceptance cuts used for the calculation of VBF (multi-)Higgs production in $p p$ collision (VBF cuts) for three different collider energies.

\begin{tabular}{lccc}
\hline \hline Cuts & $\sqrt{s}=14 \mathrm{TeV}$ & $\sqrt{s}=27 \mathrm{TeV}$ & $\sqrt{s}=100 \mathrm{TeV}$ \\
\hline$P_{t}(j)$ & $>20 \mathrm{GeV}$ & $>20 \mathrm{GeV}$ & $>30 \mathrm{GeV}$ \\
$\Delta R(j, j)$ & $>0.8$ & $>0.8$ & $>0.8$ \\
$|\eta(j)|$ & $<5.0$ & $<5.0$ & $<8.0$ \\
$\Delta \eta(j, j)$ & $>3.6$ & $>3.6$ & $>4.0$ \\
$M(j, j)$ & $>500 \mathrm{GeV}$ & $>500 \mathrm{GeV}$ & $>800 \mathrm{GeV}$ \\
\hline \hline
\end{tabular}




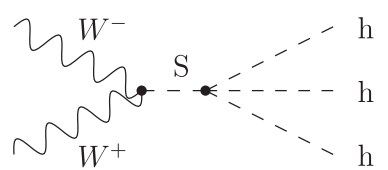

FIG. 3. Triple-Higgs production diagram with a five-point vertex $W W h h h$ effectively generated by an auxiliary field $S$.

$p p$ collider with $27 \mathrm{TeV}$ c.m. energy, and for a $100 \mathrm{TeV} p p$ collider.

For the numerical calculations, we use the automatic Monte Carlo integration and simulation packages WHIZARD 2.3 [67] and Madgraph5_aMC@NLO [68], where we have implemented the effective Lagrangian (2.1). For Madgraph5_aMC@NLO, we have constructed an appropriate Universal FeynRules Output (UFO) model file. ${ }^{2}$ For WHIZARD, we introduced an auxiliary field $S$ with a Lagrangian,

$$
\begin{aligned}
\mathcal{L}_{S}= & \frac{1}{2}\left(\partial_{\mu} S\right)^{2}-\frac{1}{2} M^{2} S^{2}-g_{S h h h}\left(\partial^{2} S\right) h^{3} \\
& +g_{W, a 3} \frac{2 m_{W}^{2}}{v^{3}} S W^{\mu} W_{\mu}-\frac{g_{W, b 3}}{v^{3}} S W^{\mu \nu} W_{\mu \nu} .
\end{aligned}
$$

Choosing $M=0$ and $g_{S h h h}=-1$ and restricting the calculation to the triple-Higgs production process, $g_{W, a 3}$ and $g_{W, b 3}$ become equivalent to the parameters in our convention, and the resulting amplitude expression is identical to the one that follows from using (2.1) directly, cf. Fig. 3. The anomalous $Z Z h h h$ vertex is implemented in a similar way.

We have cross-checked numerical results from WHIZARD and Madgraph5_aMC@NLO and found mutual agreement. As another cross-check, we have validated selected results against the package VBFNLO [84,85], again with good agreement.

For the pure SM, we obtain the cross sections after VBF cuts as listed in Table VI. All numerical results are computed at leading order in the strong and electroweak perturbative expansions.

The VBF cuts in Table $\mathrm{V}$ force the remnant jets to a forward/backward configuration, with high energy and momentum, as it is expected from $q \rightarrow W q^{\prime}$ splitting in the VBF signal region. We require $p_{T}(j)>20 \mathrm{GeV}$ for 14 and $27 \mathrm{TeV}$, and $30 \mathrm{GeV}$ for $100 \mathrm{TeV}$, respectively. Regarding the transition from LHC kinematics to a $100 \mathrm{TeV}$ collider, our numerical results demonstrate that the forward jets can acquire significantly larger rapidity than at lower energy (Fig. 4). Therefore, we assume a better rapidity coverage for the detector at $100 \mathrm{TeV}$ and have adapted our cuts in Table $\mathrm{V}$ accordingly.

\footnotetext{
${ }^{2}$ The current version 2.8 .1 of WHIZARD does support this UFO standard. The calculations for the current paper relies on an earlier version which did not support the five-point vertices in the EFT contributions to $V V \rightarrow h h h$.
}

TABLE VI. SM values for the cross sections of the processes $p p \rightarrow h j j, p p \rightarrow h h j j$, and $p p \rightarrow h h h j j$ with VBF cuts, at three different collider energies.

\begin{tabular}{lccc}
\hline \hline Process & $\sigma(14 \mathrm{TeV})[\mathrm{fb}]$ & $\sigma(27 \mathrm{TeV})[\mathrm{fb}]$ & $\sigma(100 \mathrm{TeV})[\mathrm{fb}]$ \\
\hline$p p \rightarrow h j j$ & $1.64 \times 10^{3}$ & $4.87 \times 10^{3}$ & $2.60 \times 10^{4}$ \\
$p p \rightarrow h h j j$ & 1.10 & 4.32 & 41.2 \\
$p p \rightarrow h h h j j$ & $2.73 \times 10^{-4}$ & $1.73 \times 10^{-3}$ & $4.50 \times 10^{-2}$ \\
\hline \hline
\end{tabular}

\section{A. Higgs-pair production}

The analysis of Higgs-pair production, $V V \rightarrow h h$, aims at a determination of the $V V h h$ and $h h h$ couplings. Anomalous lower-order couplings such as $V V h$ would affect the amplitudes but can be measured in lower-order processes, with considerably higher event rates. We assume that such measurements yield better precision. For the sake of simplicity, we thus fix the $V V h$ couplings to their SM values, $g_{V, a 1}=1$ and $g_{V, b 1}=0$. In addition, we assume the custodial-symmetry relations $g_{W, a 2}=g_{Z, a 2}=g_{V, a 2}$ and $g_{W, b 2}=g_{Z, b 2}=g_{V, b 2}$ whenever contributions of the $Z$ boson are considered. Furthermore, we introduce the shifts $\delta g_{V, a 2}=g_{V, a 2}-1, \quad \delta \lambda_{3}=\lambda_{3}-1$ which multiply the deviation with respect to the SM, as parameters in our calculation.

By construction, the tree-level result for the cross section in the EFT depends linearly and bilinearly on the free parameters, and can be cast into the form

$$
\begin{aligned}
& \sigma(p p \rightarrow h h j j)=\sum_{i+j+k \leq 2} \sigma_{i j k}^{h h}\left(\delta g_{V, a 2}\right)^{i} g_{V, b 2}^{j}\left(\delta \lambda_{3}\right)^{k} \\
= & \sigma_{000}^{h h}+\sigma_{100}^{h h} \delta g_{V, a 2}+\sigma_{200}^{h h}\left(\delta g_{V, a 2}\right)^{2} \\
& +\sigma_{010}^{h h} g_{V, b 2}+\sigma_{110}^{h h} \delta g_{V, a 2} g_{V, b 2}+\sigma_{020}^{h h} g_{g_{V, b 2}}^{2} \\
& +\sigma_{001}^{h h} \delta \lambda_{3}+\sigma_{101}^{h h} \delta g_{V, a 2} \delta \lambda_{3}+\sigma_{011}^{h h} g_{V, b 2} \delta \lambda_{3}+\sigma_{002}^{h h}\left(\delta \lambda_{3}\right)^{2} .
\end{aligned}
$$

In Table VII, we display the values of the SM cross section $\sigma_{000}^{h h}$ and of all EFT coefficients $\sigma_{i j k}^{h h}$, evaluated for the three collider energies of 14, 27, and $100 \mathrm{TeV}$. As discussed in Sec. III B, the amplitude contributions proportional to $\delta g_{V, a 2}$ and $g_{V, b 2}$ grow linearly with $s$. For any nonzero values of $\delta g_{V, a 2}$ or $g_{V, b 2}$, the resulting contributions to the cross section will break perturbative unitarity as $s$ increases. If such terms are present, we should expect a rapidly growing enhancement in the Higgs-pair invariantmass distribution $m(h h)$, eventually dampened by nonperturbative rescattering corrections. We will discuss this property below.

The amplitude contribution proportional to $\delta \lambda_{3}$ does not grow with energy relative to the SM cross section. The dependence on $\delta \lambda_{3}$ is dominated by the low-energy region, and constraining $\delta \lambda_{3}$ is much more challenging than constraining $\delta g_{V, a 2}$ and $g_{V, b 2}$. Conversely, the uncertainty 


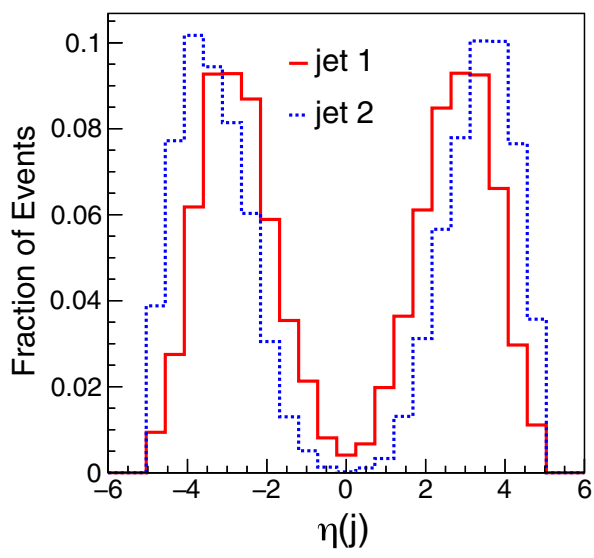

(a) $14 \mathrm{TeV}$

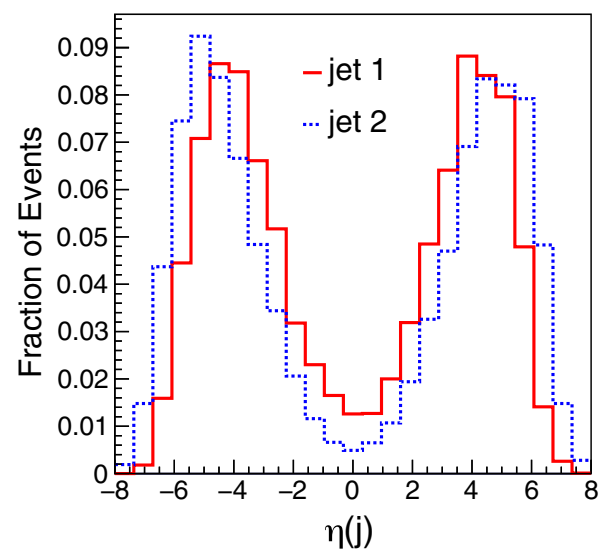

(b) $100 \mathrm{TeV}$

FIG. 4. Rapidity distribution ( $\eta$ ) of the forward tagging jets at (a) $14 \mathrm{TeV}$ and (b) $100 \mathrm{TeV}$. Jet 1 (2) labels the harder (softer) jet, respectively.

on $\delta \lambda_{3}$ is not essential for studying the dependence on $\delta g_{V, a 2}$ and $g_{V, b 2}$. In the following, we focus on the effects induced by $\delta g_{V, a 2}$ and $g_{V, b 2}$.

When adopting the EFT approach for an analysis, there is an underlying assumption that the included terms are dominant, and higher-order terms can be dropped. In a cross section calculation, the formally leading term is the interference of the linear EFT contribution with the SM part, which should be larger than the quadratic EFT contribution. In the present case, the linear coefficient $\sigma_{010}^{h h}$ is much smaller than the naive expectation $\mathcal{O}\left(\sqrt{\sigma_{000}^{h h} \sigma_{020}^{h h}}\right)$. The interference effect is suppressed, and the squared term $\sigma_{020}^{h h}$ is more important even for small values of $g_{V, b 2}$.

In fact, there is a cancellation among regions in phase space caused by a helicity mismatch between the SM and the EFT terms. The parameter $g_{V, b 2}$ multiplies the coupling between the transverse vector bosons and Higgs bosons, while $\delta g_{V, a 2}$ and the SM contribution describe the coupling between longitudinal vector bosons and Higgs bosons. Consequently, different azimuthal distributions reflect the different polarizations of intermediate vector bosons. The finite vector-boson masses induce some level of mixing, so the cancellation is not exact.

To illustrate this property, in Fig. 5 we show the distribution of $\Delta \phi(j j)$ at the $14 \mathrm{TeV}$ LHC, i.e., the azimuthal-angle correlation of the two VBF jets. For $\sigma_{000}^{h h}, \sigma_{100}^{h h}$, and $\sigma_{200}^{h h}$ terms, the distribution of $\Delta \phi(j j)$ is almost flat. By contrast, for $\sigma_{010}^{h h}, \sigma_{110}^{h h}$, and $\sigma_{020}^{h h}$, the differential cross section depends on $\Delta \phi(j j)$ with a sign flip near $\pi / 2$.

We conclude that the interference contribution of $g_{V, b 2}$, for instance, can significantly be enhanced by constructing an appropriate observable, weighing events by azimuthal distance. Furthermore, the azimuthal dependence in Fig. 5 discriminates between the two different EFT parameters and should definitely be accounted for in an analysis. Since helicity-mixing effects disappear with increasing energy, the discrimination becomes even clearer for the higher collider energies of 27 or $100 \mathrm{TeV}$.

We now turn to the distribution in the observable $m(h h)$, the invariant mass of the final state as the total c.m. energy of the elementary $V V \rightarrow h h$ scattering. In this observable, effects growing with energy are manifest, and we should study the behavior of the terms proportional to $\delta g_{V, a 2}$ and $g_{V, b 2}$.

In Fig. 6, we show the $m(h h)$ distribution of the $\sigma_{200}^{h h}$ and $\sigma_{020}^{h h}$ terms (green and blue, respectively) and the SM distribution (red). As discussed above, the EFT distributions decrease much slower with increasing $m(h h)$, compared to the SM curve. $\delta g_{V, a 2}$ and $g_{V, b 2}$ lead to similar $V V \rightarrow h h$ subamplitudes where the leading contribution for $\delta g_{V, a 2}$ corresponds to longitudinal vector bosons, while for $g_{V, b 2}$ it corresponds to transverse vector bosons. The dominant contribution to the complete off shell process originates from quasi-on-shell collinear splitting $q \rightarrow V q^{\prime}$. The emission of longitudinal vector boson from a quark is kinematically suppressed in relation to transverse vector bosons [86]. Consequently, for comparable values of the

TABLE VII. Coefficients $\sigma_{i j k}^{h h}$ (in fb) in the expression (4.2) for VBF $h h$ at three different collider energies.

\begin{tabular}{lcccccccccc}
\hline \hline & $\sigma_{\mathrm{SM}}^{h h}=\sigma_{000}^{h h}$ & $\sigma_{100}^{h h}$ & $\sigma_{200}^{h h}$ & $\sigma_{010}^{h h}$ & $\sigma_{110}^{h h}$ & $\sigma_{020}^{h h}$ & $\sigma_{001}^{h h}$ & $\sigma_{101}^{h h}$ & $\sigma_{011}^{h h}$ & $\sigma_{002}^{h h}$ \\
\hline $14 \mathrm{TeV}$ & 1.10 & -3.51 & 11.0 & 1.31 & 1.7 & 87.8 & -0.81 & 3.6 & 0.35 & 0.66 \\
$27 \mathrm{TeV}$ & 4.32 & -15.0 & 61.1 & 6.91 & 9.6 & 957 & -2.89 & 14.1 & 1.4 & 2.3 \\
$100 \mathrm{TeV}$ & 41.2 & -158 & 1302 & 79.2 & 95 & $4.80 \times 10^{4}$ & -21.8 & 123 & 11.2 & 16.9 \\
\hline \hline
\end{tabular}



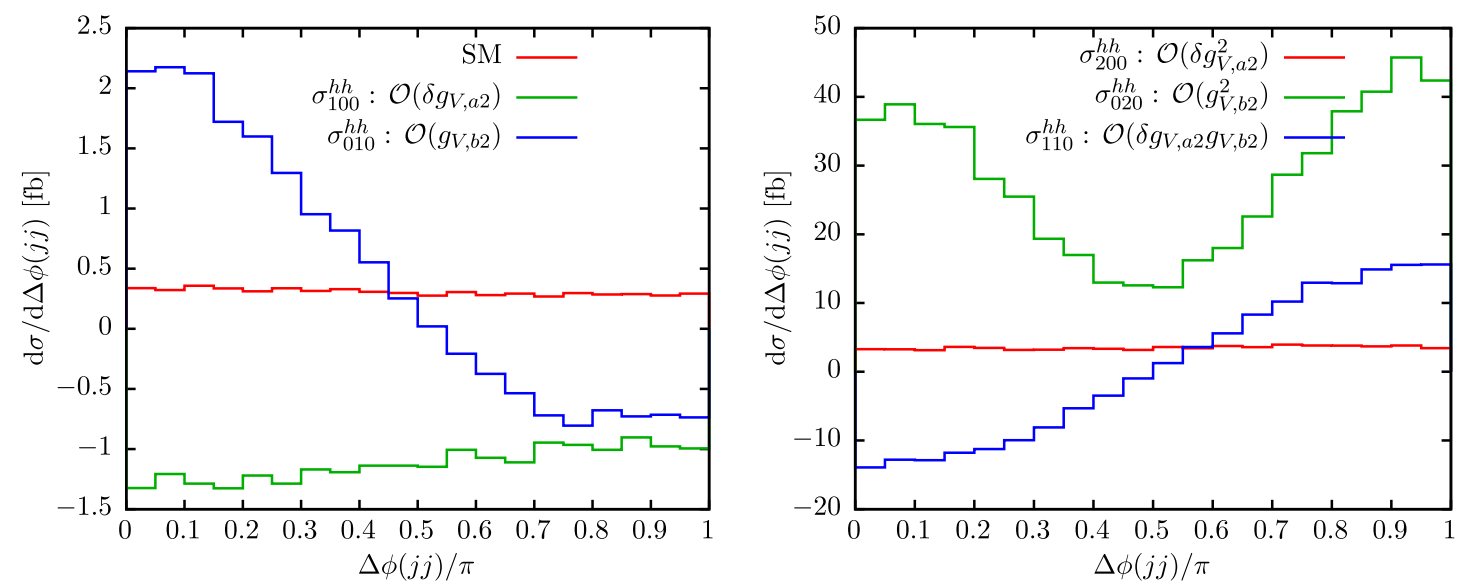

FIG. 5. Distribution of $\Delta \phi(j j)$ for various modifications of VBF $h h$ production in the EFT at the $14 \mathrm{TeV}$ LHC.

EFT coefficients $g_{V, b 2}$ and $\delta g_{V, a 2}$, the contribution from $g_{V, b 2}$ is much larger than $\delta g_{V, a 2}$ and exhibits a slower decrease as a function of $m(h h)$.

The powerlike increasing EFT distributions will leave the perturbative regime beyond some point in the highenergy region that depends on the parameters, invalidating the EFT as a systematic approximation to the (unknown) true amplitudes. Given numerical values for the EFT parameters, perturbative unitarity thus provides an upper bound on the range of validity of the EFT. We may require that the EFT should be valid up to some scale $Q$, in the range $m(h h) \leq Q$. The perturbative unitarity constraints derived in Sec. III B then provide bounds on the values of $\delta g_{V, a 2}$ and $g_{V, b 2}$. Restricting $\delta g_{V, a 2}$ and $g_{V, b 2}$ to vary only within this parameter range, we can compute the differential cross section $\frac{\mathrm{d} \sigma}{\mathrm{d} m(h h)}$ in the region $m(h h) \leq Q$, and derive the maximally allowed deviation at any point within that region.

Explicitly, we model the deviation from the SM by linear and quadratic terms in the EFT parameters, dropping any higher-dimensional contributions. Allowing a single parameter, say, $\delta g_{V, a 2}$, to deviate from zero and imposing the unitarity bound for the entire $m(h h)$ distribution below the scale $Q$, we obtain the formal condition

$$
\begin{aligned}
& \left.\max _{\delta g_{V, a 2}} \frac{\mathrm{d} \sigma^{\mathrm{NP}}}{\mathrm{d} m h)}\right|_{m(h h)=Q} \\
& =\max _{\delta g_{V, a 2}}\left|\delta g_{V, a 2}\left(\left.\frac{\mathrm{d} \sigma_{100}^{h h}}{\mathrm{~d} m(h h)}\right|_{m(h h)=Q}+\left.\delta g_{V, a 2} \frac{\mathrm{d} \sigma_{200}^{h h}}{\mathrm{~d} m(h h)}\right|_{m(h h)=Q}\right)\right|
\end{aligned}
$$

Here, we can take the unitarity bounds from Sec. III B,

$$
-\frac{8 \sqrt{2} \pi v^{2}}{Q^{2}} \leq \delta g_{V, a 2} \leq \frac{8 \sqrt{2} \pi v^{2}}{Q^{2}}
$$

Analogous results hold for $g_{V, b 2}$ if varied on its own. Regarding the general case of both $V V h h$ couplings nonzero, we recall that their interference is small and asymptotically suppressed, as discussed above. In effect, the overall deviation of the distribution is approximately the

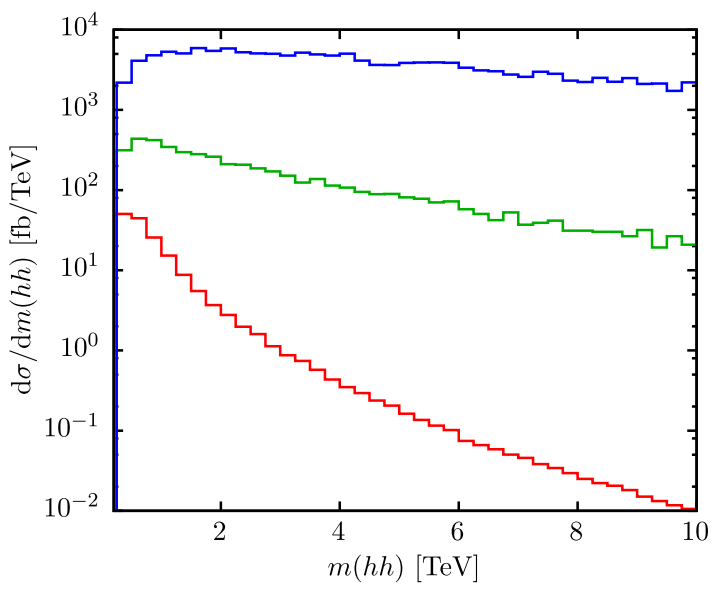

FIG. 6. Higgs-pair invariant-mass distribution for the coefficients of the leading power corrections to VBF $h h h$ production in $p p$ collisions. The SM baseline is drawn in red. We display results for $14 \mathrm{TeV}$ (left) and for $100 \mathrm{TeV}$ (right). 

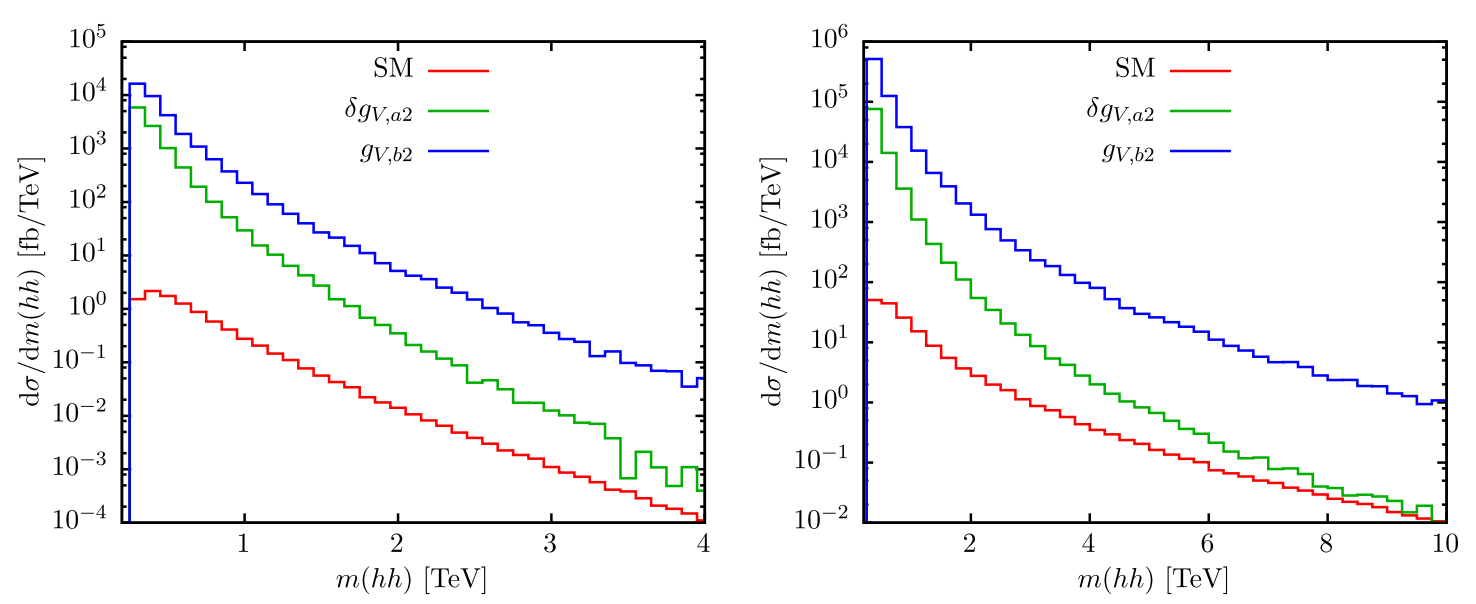

FIG. 7. Maximally allowed enhancement of the differential cross section of VBF $h h$ production in $p p$ collisions, as a function of the Higgs-pair invariant mass. We display results for $14 \mathrm{TeV}$ (left) and for $100 \mathrm{TeV}$ (right), identifying $m(h h)$ with the cutoff scale $Q$.

sum of the maximally allowed deviations from $\delta g_{V, a 2}$ and $g_{V, b 2}$ being varied separately.

In Fig. 7, we display the results following from that argument, namely the curves of maximal event rate. Taking the conditions (4.4), (4.5) literally, we obtain an upper bound on the $m(h h)$ distribution for all energies, where we identify $m(h h)$ with $Q$. We observe that there is more freedom for enhancement in the direction of $\delta g_{V, b 2}$, which corresponds to transverse gauge bosons interacting with a Higgs pair, than for $\delta g_{V, a 2}$ which parametrizes the longitudinal coupling.

\section{B. Triple-Higgs production}

We now consider the $h h h j j$ final state which contains $V V \rightarrow h h h$ as the relevant sub-amplitude, on the same footing as the $h h j j$ final state in the previous subsection. The Feynman diagrams are displayed in Fig. 2. In analogy with $V V \rightarrow h h$, we assume that $g_{W, a 1}, g_{W, a 2}, \lambda_{3}$ and $g_{W, b 1}$, $g_{W_{b} 2}, \kappa_{5}$ have been sufficiently well determined from lower-multiplicity measurements, single-Higgs and double-Higgs production in particular. For simplicity, we set these parameters to their SM values 1 and 0 , respectively.

Expanding the full dependence on the remaining free parameters, we can write the cross section as

$$
\begin{aligned}
& \sigma(p p \rightarrow h h h j j)=\sum_{i+j+k \leq 2} \sigma_{i j k}^{h h h} g_{V, a 3}^{i} g_{V, b 3}^{j}\left(\delta \lambda_{4}\right)^{k} \\
= & \sigma_{000}^{h h h}+\sigma_{100}^{h h h} g_{V, a 3}+\sigma_{200}^{h h h}\left(g_{V, a 2}\right)^{2} \\
& +\sigma_{010}^{h h h} g_{V, b 3}+\sigma_{110}^{h h h} g_{V, a 3} g_{V, b 3}+\sigma_{020}^{h h h} g_{V, b 3}^{2} \\
& +\sigma_{001}^{h h h} \delta \lambda_{4}+\sigma_{101}^{h h h} g_{V, a 3} \delta \lambda_{4}+\sigma_{011}^{h h h} g_{V, b 3} \delta \lambda_{4}+\sigma_{002}^{h h h}\left(\delta \lambda_{4}\right)^{2} .
\end{aligned}
$$

We have computed the $\sigma_{i j k}^{h h h}$ coefficients by numerical integration using WHIZARD. The results are listed in Table VIII.

Only the couplings to vector bosons, $g_{V, a 3}$ and $g_{V, b 3}$, lead to unitarity violation at high energy in the EFT calculation. Here, $g_{V, a 3}$ couples a pair of longitudinal vector bosons to the Higgs triplet, while $g_{V, b 3}$ multiplies the triple-Higgs coupling of transverse vector bosons. By contrast, a deviation in the quartic Higgs coupling $\delta \lambda_{4}$ does not lead to a powerlike enhancement. Asymptotically, its effect is subleading compared to $g_{V, a 3}$ and $g_{V, b 3}$, and we expect significantly more resolution power for the latter parameters.

Of course, the main goal of such a measurement would be to get a handle on the quartic Higgs self-coupling.

TABLE VIII. Coefficients $\sigma_{i j k}^{h h}$ (in fb) in the expression (4.6) for the process VBF $h h h$ at three different collider energies.

\begin{tabular}{lccccc}
\hline \hline$[\mathrm{fb}]$ & $\sigma_{\mathrm{SM}}^{\text {hhh }}=\sigma_{000}^{\text {hhh }}$ & $\sigma_{100}^{\text {hhh }}$ & $\sigma_{200}^{\text {hhh }}$ & $\sigma_{010}^{\text {hhh }}$ & $\sigma_{110}^{\text {hhh }}$ \\
\hline $14 \mathrm{TeV}$ & $2.792 \times 10^{-4}$ & $-5.21 \times 10-4$ & 0.146 & $2.44 \times 10^{-3}$ & $1.55 \times 10^{-2}$ \\
$27 \mathrm{TeV}$ & $1.66 \times 10^{-3}$ & $-1.45 \times 10^{-3}$ & 2.30 & $2.00 \times 10^{-2}$ & 0.195 \\
$100 \mathrm{TeV}$ & $3.10 \times 10^{-2}$ & $5.18 \times 10^{-2}$ & 495 & 0.398 & 11 \\
{$[\mathrm{fb}]$} & $\sigma_{020}^{\text {hhh }}$ & $\sigma_{001}^{\text {hhh }}$ & $\sigma_{101}^{\text {hhh }}$ & $\sigma_{011}^{\text {hhh }}$ & $\sigma_{002}^{\text {hh }}$ \\
$14 \mathrm{TeV}$ & 3.29 & $-8.32 \times 10^{-5}$ & $7.24 \times 10^{-3}$ & $4.7 \times 10^{-4}$ & $2.09 \times 10^{-4}$ \\
$27 \mathrm{TeV}$ & 121 & $-3.55 \times 10^{-4}$ & $5.28 \times 10^{-2}$ & $2.9 \times 10^{-3}$ & $1.06 \times 10^{-3}$ \\
$100 \mathrm{TeV}$ & $6.67 \times 10^{4}$ & $-3.07 \times 10^{-3}$ & 1.61 & $4.6 \times 10^{-2}$ & $1.38 \times 10^{-2}$ \\
\hline \hline
\end{tabular}



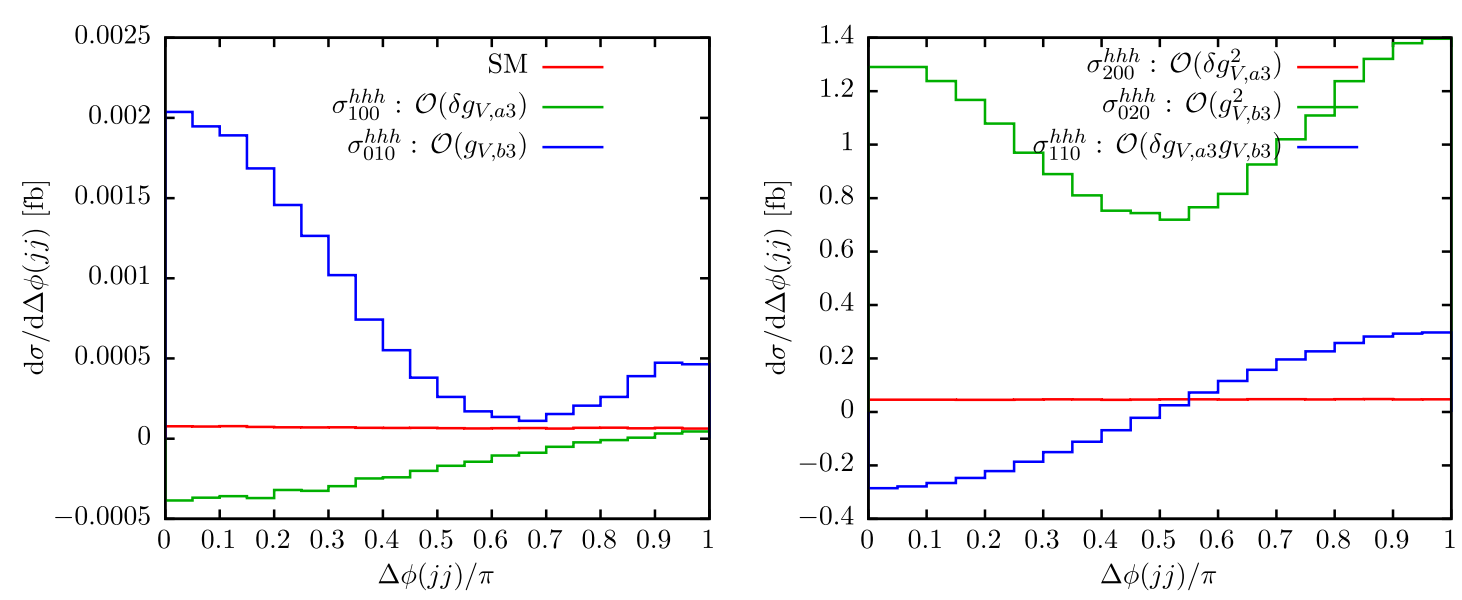

FIG. 8. Distribution of $\Delta \phi_{j j}$ for various modifications of VBF $h h h$ production at the $14 \mathrm{TeV} \mathrm{LHC}$.

The apparent dominance of the other EFT couplings makes it even more important to understand their impact on the process.

In Fig. 8, we show the azimuthal $\Delta \phi_{j j}$ distributions for VBF to $h h h$ at the $14 \mathrm{TeV}$ LHC. For the 27 and $100 \mathrm{TeV}$ colliders, the results are similar. The behavior is superficially analogous to the double-Higgs case, taking finitemass effects into account. Apart from the much smaller absolute values, there also are significant differences. The Lorentz structure of the interference terms is more complicated. This is reflected in the $\sigma_{100}^{h h h}$ and $\sigma_{010}^{h h h}$ distributions.

To examine the energy dependence of the $g_{V, a 3}$ and $g_{V, b 3}$ contributions, in Fig. 9 we plot the appropriate $m(h h h)$ distributions. While the SM curve displays the expected decrease with increasing $m(h h h)$, the $\mathcal{O}\left(g_{V, a 3}^{2}\right)$ and $\mathcal{O}\left(g_{V, b 3}^{2}\right)$ coefficients exhibit a wide plateau with a very slow falloff for high masses.

This behavior demonstrates the necessity of considering unitarity constraints on the EFT parameters. Based on formulas given in Sec. III C with a cutoff $Q, g_{V, a 3}$ and $g_{V, b 3}$ are restricted to the range

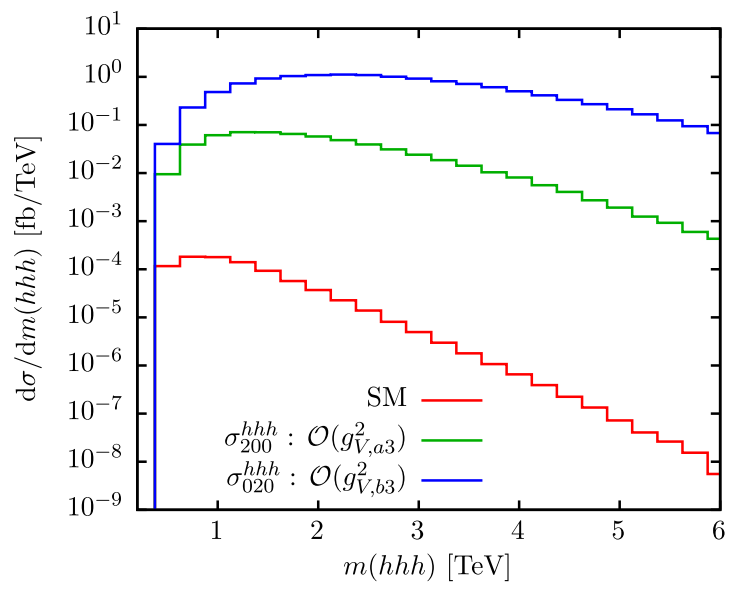

$$
\left|g_{V, a 3}\right|,\left|g_{V, b 3}\right|<\frac{64 \sqrt{3} v^{3} \pi^{2}}{Q^{3}} .
$$

In analogy with the double-Higgs case discussed before, we use this relation to deduce the maximally allowed enhancement of the differential cross section as a function of $m(h h h)$, varying either $g_{V, a 3}$ or $g_{V, b 3}$. The results are presented in Fig. 10. As the interference is negligible, we may sum the two sources incoherently to obtain an absolute upper bound. We conclude that the potential enhancement is substantial in the transverse mode, somewhat less so in the longitudinal mode. This conclusion is qualitatively similar to the double-Higgs case, cf. Fig. 7.

While we do not attempt a full study of the collider sensitivity to the final state in this work, we may remark that the presence of one extra Higgs boson relative to $h h$, reduces the signal reconstruction efficiency but also helps to suppress backgrounds. Despite the tiny cross section, obtaining some experimental limits on the parameters $g_{V, a 3}$ and $g_{V, b 3}$ may become feasible. This, in turn, would help to set meaningful bounds on the actual quartic Higgs selfcoupling.

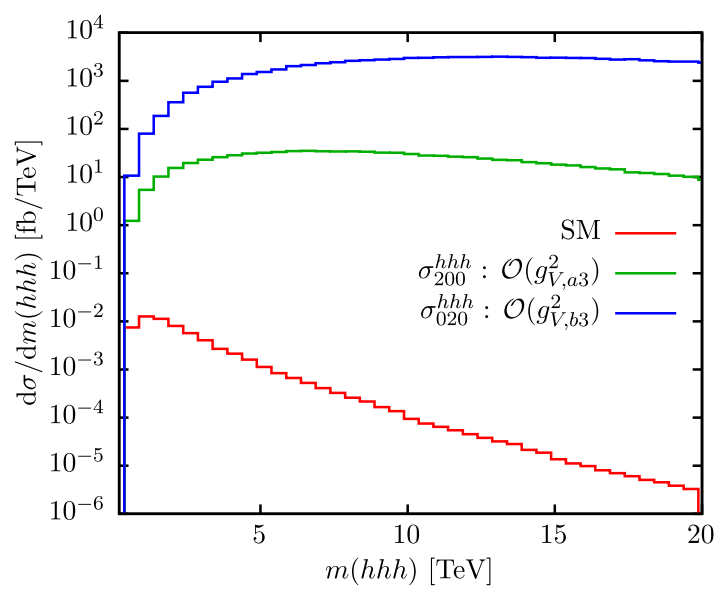

FIG. 9. Triple-Higgs invariant mass distribution for the coefficients of the leading power corrections to VBF $h h h$ production in $p p$ collisions. We display results for $14 \mathrm{TeV}$ (left) and for $100 \mathrm{TeV}$ (right). 

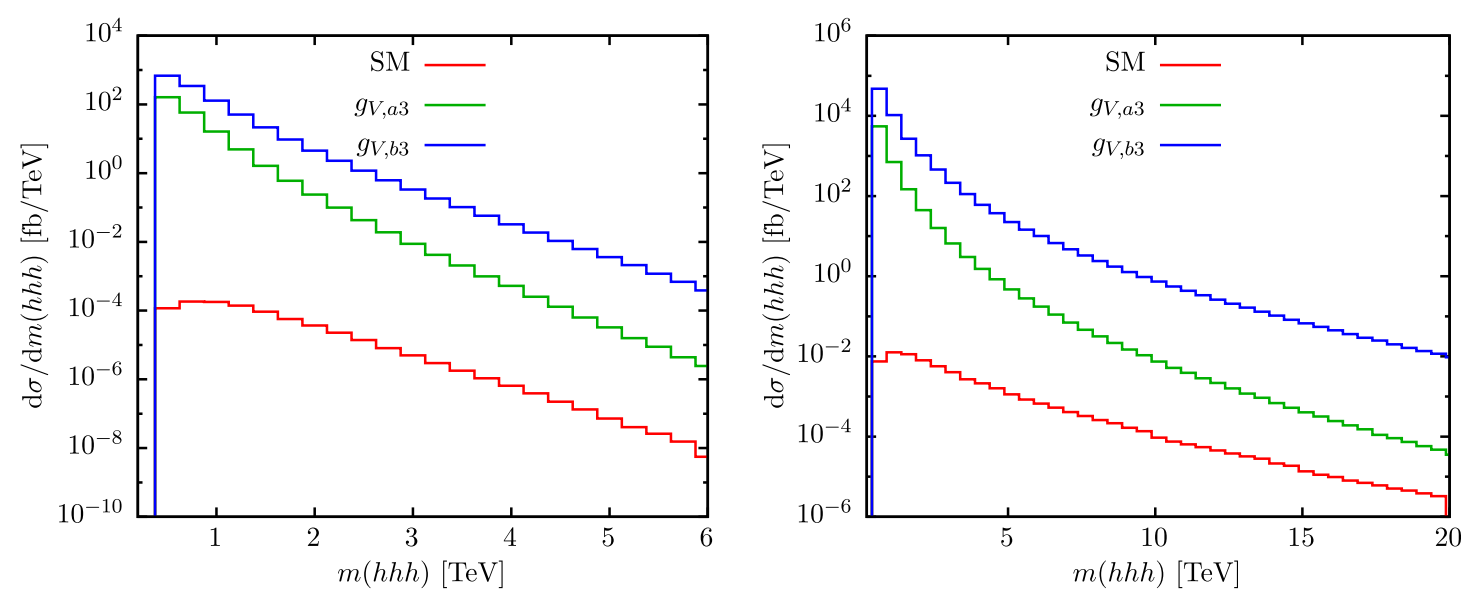

FIG. 10. Maximally allowed enhancement of the differential cross section of VBF $h h h$ production in $p p$ collisions, as a function of the triple-Higgs invariant mass. We display results for $14 \mathrm{TeV}$ (left) and for $100 \mathrm{TeV}$ (right), identifying $m(h h h)$ with the cutoff $Q$.

\section{Unitarity bounds for the cross sections of multi-Higgs final states}

We study the relation of the indirect and direct discovery potential for the two-Higgs process at the three collision energies $\sqrt{s}=14,27$, and $100 \mathrm{TeV}$. The criterion is whether for some given parameter point in the EFT, a significant part of the extrapolated cross section violates the (parameter-dependent) unitarity limit, and thus gives access to a range where nonEFT corrections must become dominant. To this end, in Fig. 11, we plot the invariant-mass distribution of the process $p p \rightarrow h h j j$. We fix the EFT parameters to $g_{a 2}=$ 0.9 (left plot) and $g_{b 2}=0.1$ (right plot), respectively. For these values, unitarity is saturated in the distribution at $4.6 \mathrm{TeV}$. To the left of that cutoff, the curves should extrapolate the low-energy effective theory in a still meaningful way. To the right, the extrapolation loses its validity, and the actual result will look significantly different. We expect nonperturbative suppression, resonances, or other new effects to set in at or before this effective cutoff. Stated differently, given a nonzero parameter value which by itself is an indirect sign of physics beyond the SM, the distribution probes a range where direct manifestations of new physics appear.

For the LHC energy $\sqrt{s}=14 \mathrm{TeV}$ and $g_{a 2}=0.9$, the cross section in the range $m(h h)>4.6 \mathrm{TeV}$ is negligible. There is no direct sensitivity to new effects in this process. Increasing the collision energy to $\sqrt{s}=27 \mathrm{TeV}$, the integrated cross section with $m(h h)>4.6 \mathrm{TeV}$ provides a fraction of $0.2 \%$ of the total cross section. If we look at $\sqrt{s}=100 \mathrm{TeV}$, the fraction of events with $m(h h)>$ 4.6 $\mathrm{TeV}$ provides $4.2 \%$. Assuming that the differential distribution is accessible in an experiment, for sufficiently large luminosity, a separation and discovery of new effects beyond the EFT becomes feasible. The analysis clearly benefits from an increase collision energy.
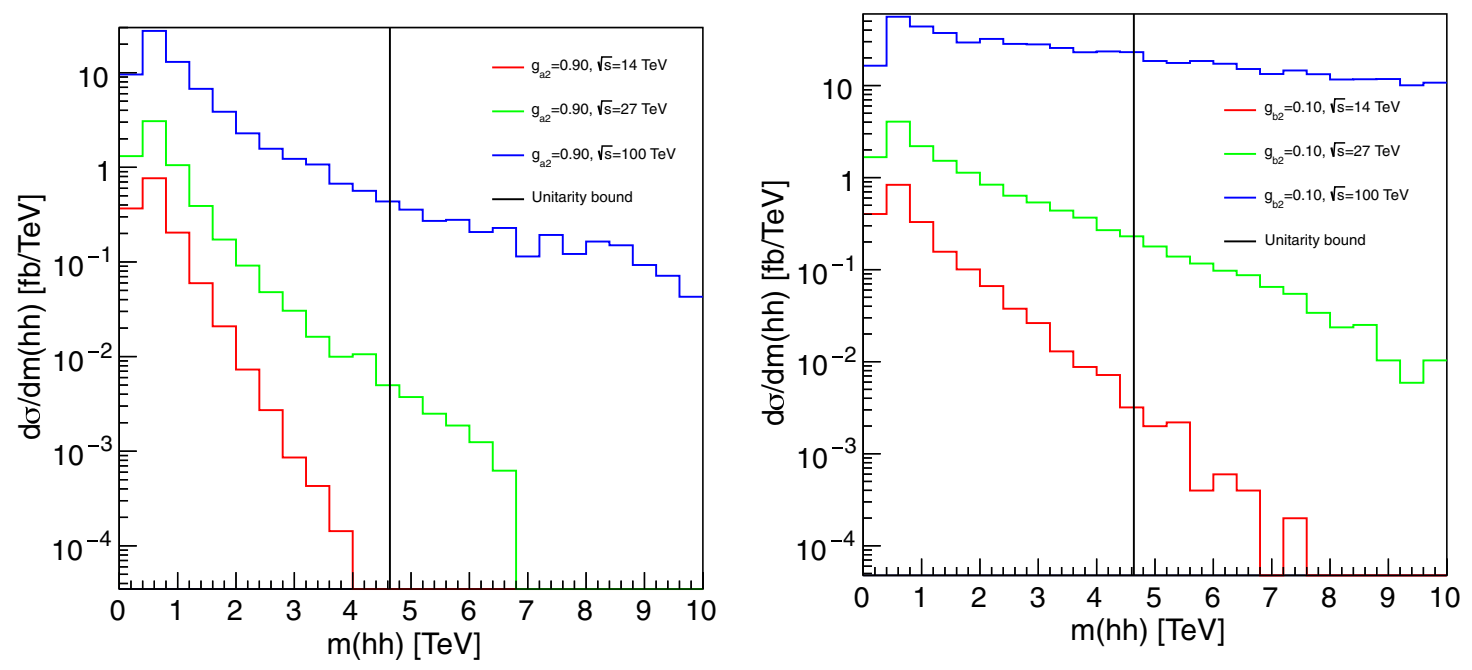

FIG. 11. The unitarity bounds and discovery potentials of different collision energy at hadronic colliders, i.e., 14, 27 and $100 \mathrm{TeV}$, are shown for $p p \rightarrow h h j j$ process. 

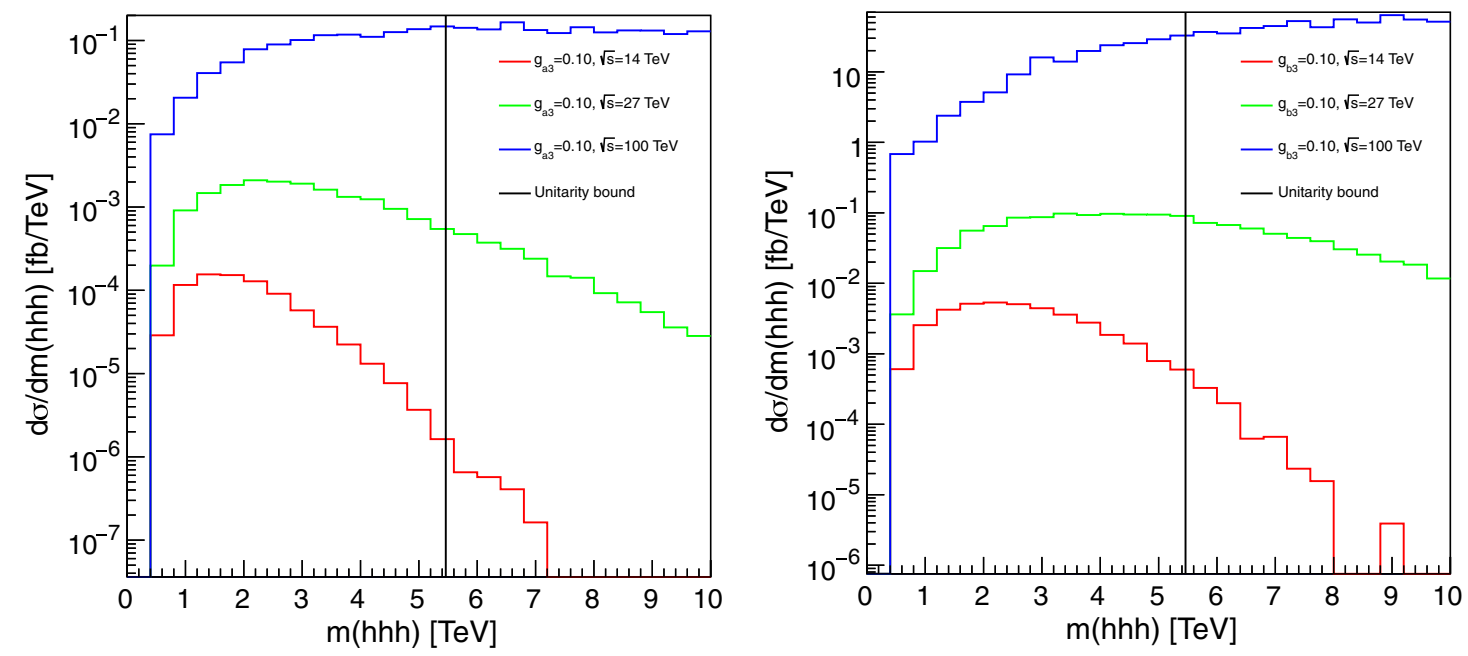

FIG. 12. The unitarity bounds and discovery potentials of different collision energy at hadronic colliders, i.e., 14, 27, and 100 TeV, are shown for $p p \rightarrow h h h j j$ processes.

The situation for the $g_{b 2}=0.10$ case is qualitatively similar. Due to the larger incoming flux of transverse polarized $\mathrm{W}$ bosons, the integrated cross section beyond the limit of $4.6 \mathrm{TeV}$ amounts to fractions $0.3 \%, 6.6 \%$, and $48.0 \%$ of the total rate at 14,27 , and $100 \mathrm{TeV}$, respectively. While the range of validity of the EFT is similar for both parameter points, the $g_{b 2}$ direction grants a more favorable view at new effects than $g_{a 2}$.

In Fig. 12, we analogously study the triple-Higgs process $p p \rightarrow h h h j j$. The parameter $g_{a 3}=0.1$ for the left plot $\left(g_{b 3}=0.1\right.$ for the right plot) corresponds to a cutoff at $5.42 \mathrm{TeV}$. Again, increasing the collider energy enhances the fraction of the cross section that falls beyond the unitarity limit in a naive extrapolation and thus is subject to nonperturbative corrections and new effects. In fact, for all parameter values, the distribution is more spread out toward higher invariant masses, compared to the doubleHiggs production channel. For instance, if the collision energy is $\sqrt{s}=100 \mathrm{TeV}$, the rate beyond the limit of $m(h h h)>5.42 \mathrm{TeV}$ becomes the dominant fraction in the extrapolation.

While a significant influence of non-EFT new effects is an interesting property of the naive extrapolation, we also have to take account of the fact that the true rate cannot exceed the unitarity limit. If we interpret this limit in the mass distribution as an ultimate cutoff, by removing the part of the cross section beyond that limit we can estimate the maximal cross section which is actually allowed by unitarity, for any given collision energy.

In Fig. 13, we show the maximally allowed cross sections as a function of the collider energy for $p p \rightarrow h h j j$ and $p p \rightarrow h h h j j$, respectively, denoted by the black curves.

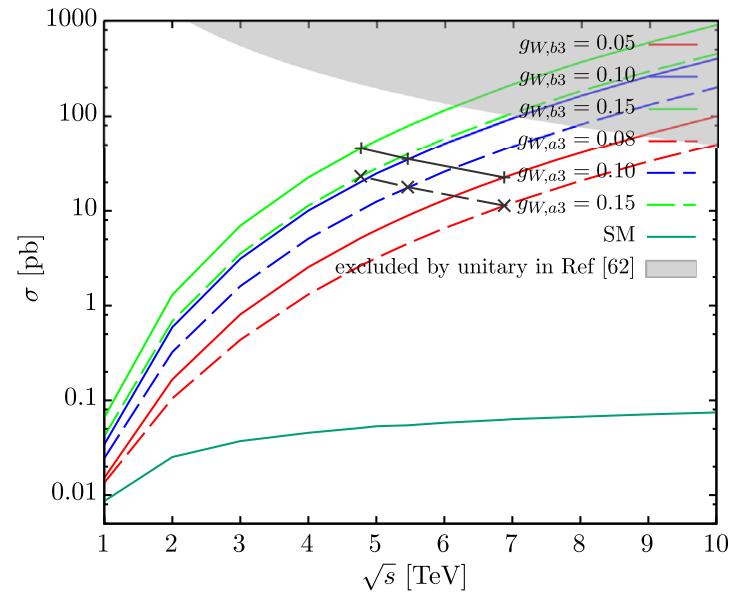

FIG. 13. Total cross section of $p p \rightarrow h h j j$ and $p p \rightarrow h h h j j$ as a function of the total energy, for three exemplary values of the free parameters. The black curves indicate the unitarity bounds for the total cross section as a function of the energy (see the text for details). For the purpose of comparison, we also show the cross section of the SM, and the unitarity bounds from Ref. [62], represented by the shaded regions. 
To avoid unnecessary complexity, we read off the black curves from the cross-section predictions for specific values of $g_{W, a 2}\left(g_{W, b 2}, g_{W, a 3}\right.$, and $\left.g_{W, b 3}\right)$. In principle, by using the steps given below, we can determine the maximal cross section for any value of $s$.

In the left plot of Fig. 13, we present the maximal cross sections for $p p \rightarrow h h j j$. For the purpose of comparison, the shaded regions indicate the parameter space which is excluded by unitarity if we use the method given in the Ref. [62].

To show how the constraints for $2 \rightarrow 2$ processes given in Eqs. (3.22)-(3.24) in our method can determine the maximal cross section, we have selected three values of $g_{W, a 2}\left(g_{W, b 2}\right)=0.15,0.10$, and 0.05 , which correspond to three UV cutoffs $3.5,4.2$, and $6.5 \mathrm{TeV}$, respectively. Accordingly, by using the formula given in Eq. (4.3), we generate three points where the dashed (solid) lines intersect the extrapolated cross section curves for $g_{W, a 2}$ $\left(g_{W, b 2}\right)$. Without any constraint from unitarity, the cross extrapolated cross sections would further increase with the increase of collision energy, crossing the black lines and ultimately entering the shaded region.

If we consider the triple-Higgs production process (right plot of Fig. 13), we arrive at similar conclusions.

There are the following three comments on this plot:

(i) The unitarity bounds that correspond to nontrivial values of $g_{W, a 2}$ and $g_{W, b 2}$ differ from each other: the applicable bound depends on the location in the EFT parameter space. For example, when the cutoff is taken as $3.5 \mathrm{TeV}$, the maximal cross section is 30 and $80 \mathrm{pb}$, respectively. This is a natural result due to the different incident flux of polarized vector bosons in the protons, as can be read out from Eq. (4.3).

(ii) The unitarity bounds derived in our method are more strict than the method determined in Ref. [62]. For example, when the cutoff is taken as $3.5 \mathrm{TeV}$, the maximal cross section is $300 \mathrm{pb}$ in term of the method of Ref. [62], which is different from the values in our method given above.

(iii) The SM cross section curves for these two subprocesses are also displayed in Fig. 13. We note that the maximally allowed cross section decreases with the increase of the total collision energy, but remains larger than that of the SM. In the small $s$ region $(s \sim 3-4 \mathrm{TeV})$, the allowed rate for the process $p p \rightarrow h h j j$ can be 1-2 orders of magnitude larger than that of the SM. For the process $p p \rightarrow h h h j j$, in the same region, the allowed rate can be 3-4 orders of magnitude larger than that of the SM.

For example, for the cutoff $s=4.7 \mathrm{TeV}$, the maximally allowed cross section for $g_{W, b 3}=0.15$ is $45 \mathrm{pb}$, while for $g_{W, a 3}=0.15$ we obtain $25 \mathrm{pb}$. The method given in Ref. [62] yields a weaker limit of around $200 \mathrm{pb}$.
For example, for the cutoff $s=4.7 \mathrm{TeV}$, the maximal cross section for $g_{W, b 3}=0.15$ is $45 \mathrm{pb}$ or so, while the one for $g_{W, a 3}=0.15$ is about $25 \mathrm{pb}$. In contrast, the results given in Ref. [62] (which are a direct use of the unitary condition $\sigma(2 \rightarrow n)<\frac{4 \pi}{s}$ from the methods given in $[73,74]$ ) yields a number $200 \mathrm{pb}$ or so.

To appreciate the difference between the results given in [62] and our results, it is noticed that the information of the helicity amplitudes given in Eqs. (3.22)-(3.24) and Eqs. (3.28)-(3.30) play a central role.

\section{Bounds on multi-Higgs production in the SILH and Higgs-inflation models}

After discussing unitarity bounds in the generic EFT context, we apply the formalism to the more specific SILH (Sec. A) and Higgs-inflation (Sec. A 1) models. We recall from Sec. A that the SILH model describes a truncation of the EFT expansion in a linear gauge representation, such that interactions with different multiplicities become related to each other. The Higgs-inflation model is even more restricted, and all corrections depend on just a single parameter.

For the SILH model, we simplify the treatment by considering only $c_{W}$ and $c_{B}$ as nonzero parameters, and furthermore impose the relation $c_{B}=-c_{W}$ to ensure that the $\hat{S}$ parameter is zero at tree level, cf. Eq. (A8). Denoting $\hat{c}_{W}=c_{W} \frac{m_{W}^{2}}{m_{\rho}^{2}}$ and $\hat{c}_{Z}=\left(1-\tan ^{2} \theta_{W}\right) \hat{c}_{W} \approx 0.71 \hat{c}_{W}$, we have $\delta g_{W, a 2}=3 \delta g_{W, a 1}=-3 \hat{c}_{W}, \quad \delta g_{Z, a 2}=3 \delta g_{Z, a 1}=3 \hat{c}_{Z}$.

Evaluating the unitarity constraints given in Sec. III for the $W$ fusion subamplitudes in this parametrization, we obtain

$$
\begin{gathered}
b_{0}^{W^{+} W^{-} \rightarrow h h}(00)=\frac{s^{2}}{2^{9} \pi^{2} v^{4}}\left(\hat{c}_{W}-\hat{c}_{W}^{2}\right)^{2} \leq \frac{1}{4}, \\
b_{0}^{W^{+} W^{-} \rightarrow h h h}(00)=\frac{s^{3}}{3 \times 2^{10} \pi^{4} v^{6}}\left(1+\hat{c}_{W}\right)^{2}\left(\hat{c}_{W}-\hat{c}_{W}^{2}\right)^{2} \leq \frac{1}{4} .
\end{gathered}
$$

If we assume that $\delta g_{W, a 1} \ll 1$, we can ignore higher-order terms in $\delta g_{W, a 1}$ and reduce this to

$$
\begin{gathered}
b_{0}^{W^{+} W^{-} \rightarrow h h}(00) \approx \frac{s^{2}}{2^{9} \pi^{2} v^{4}} \hat{c}_{W}^{2} \leq \frac{1}{4}, \\
b_{0}^{W^{+} W^{-} \rightarrow h h h}(00) \approx \frac{s^{3}}{3 \times 2^{10} \pi^{4} v^{6}} \hat{c}_{W}^{2} \leq \frac{1}{4} .
\end{gathered}
$$

Comparing the constraints from the double- and tripleHiggs production processes, we find that once $\sqrt{s} \geq$ $\sqrt{6} \pi v \approx 1.9 \mathrm{TeV}$, the $b_{0}$ value that results from VBF triple-Higgs production is larger than its double-Higgs 
TABLE IX. Coefficients $\sigma_{j}^{h h}$ (in fb) in Eq. (4.14) for VBF $h h$ in SILH with $c_{B}=-c_{W}$. We show values for $p p$ collisions at 14 , 27 , and $100 \mathrm{TeV}$, respectively.

\begin{tabular}{lccccc}
\hline \hline$[\mathrm{fb}]$ & $\sigma_{\mathrm{SM}}^{h h}=\sigma_{0}^{h h}$ & $\sigma_{1}^{h h}$ & $\sigma_{2}^{h h}$ & $\sigma_{3}^{h h}$ & $\sigma_{4}^{h h}$ \\
\hline $14 \mathrm{TeV}$ & 1.10 & 0.90 & 10.6 & -9.3 & 16.1 \\
$27 \mathrm{TeV}$ & 4.32 & 3.0 & 56.4 & -64.5 & 78.1 \\
$100 \mathrm{TeV}$ & 41.2 & 14.3 & $1.13 \times 10^{3}$ & $-1.79 \times 10^{3}$ & $1.29 \times 10^{3}$ \\
\hline \hline
\end{tabular}

counterpart, and thus provides a stronger constraint. In addition, considering that the differential cross section is proportional to $b$ [see Eq. (B15)], this observation suggests that the cross section deviation from the SM prediction would be larger for triple-Higgs than for double-Higgs production. The triple-Higgs production process should be considered as supplementing relevant information, provided the difficulties of isolating the final state can be overcome.

We expand the cross section for VBF double-Higgs production in the form

$$
\sigma(p p \rightarrow h h j j)=\sum_{j=0}^{4} \sigma_{j}^{h h} \hat{c}_{W}^{j}
$$

and list the coefficient values in Table IX. In contrast to the generic EFT (2.1) where all distinct interactions have distinct coefficients, the SILH parameters enter the amplitude quadratically, and thus appear with up to fourth power in the cross section.

We observe that $\sigma_{2}^{h h} \gg \sigma_{1}^{h h}$, in line with the discussion in the preceding subsections. On the other hand, $\sigma_{2}^{h h}, \sigma_{3}^{h h}, \sigma_{4}^{h h}$ are all of the same order; they are accounted for in the $b_{0}$ term in Eq. (4.10). As long as $\hat{c}_{W} \ll 1$, the contributions from $\sigma_{3}^{h h}$ and $\sigma_{4}^{h h}$ are much smaller than the one from $\sigma_{2}^{h h}$.

Similarly, for VBF triple-Higgs production, the cross section becomes

$$
\sigma=\sum_{j=0}^{6} \sigma_{j}^{h h h} \hat{c}_{W}^{2},
$$

and the numerical results are shown in Table X. As expected, from the leading contribution represented by Eq. (4.11), we find that $\left(\left|\sigma_{0}^{h h h}\right|,\left|\sigma_{1}^{h h h}\right|,\left|\sigma_{3}^{h h h}\right|,\left|\sigma_{5}^{h h h}\right|\right) \ll$ $\left(\sigma_{2}^{h h h}|,| \sigma_{4}^{h h h}|,| \sigma_{6}^{h h h} \mid\right)$. Therefore, in the case $\hat{c}_{W} \ll 1, \sigma_{j}^{h h h}$ contributions with $j>2$ are negligible with respect to the $\sigma_{2}^{\text {hhh }}$ contribution.

In Fig. 14, we present the maximally allowed enhancement with respect to the SM differential cross section for VBF double-Higgs and triple-Higgs production, respectively, after taking into account all unitarity constraints for $W^{+} W^{-} \rightarrow h h, W^{+} W^{-} \rightarrow h h h, Z Z \rightarrow h h$, and $Z Z \rightarrow h h h$. The SM cross section for VBF triple-Higgs production is indeed tiny, 2-3 orders smaller than the cross section for VBF double-Higgs production. However, if we push the SILH parameters to their unitarity limits, the differential cross section for both processes becomes much larger than the SM cross section. In fact, the enhancement of VBF triple-Higgs production can amount to 3-6 orders of magnitude, becoming comparable to VBF double-Higgs production. In the region of large multi-Higgs invariant mass, the former rate can even surpass VBF double-Higgs production. This is due to the fact that the $V V \rightarrow h h h$ cross section grows faster than $V V \rightarrow h h$, as indicated by the $b_{0}$ values given in Eqs. (4.10) and (4.11).

It is obvious that in such a region of strong interactions, the SILH model as a truncated EFT will most likely be inappropriate, and higher-order terms and nonperturbative effects will dominate the rates. Nevertheless, this particular example should serve to illustrate the generic property of strongly interacting quantum field theories, that multiple production of particles is no more suppressed relative to low-multiplicity processes, and only inclusive observables are under control. This observation holds as soon as the masses of the involved particles - in our case, the Higgs boson-become negligible. If strong interactions are a possibility, setting bounds on high-multiplicity final states is of major physical relevance even if the process itself cannot be detected.

Turning to the one-parameter Higgs-inflation model as described in Sec. A 1 , we denote $\hat{x}=6 \xi^{2} v^{2} / M_{p}^{2}$ and express the unitarity constraints given in Sec. III as

$$
\begin{gathered}
b_{0}^{W^{+} W^{-} \rightarrow h h}(00)=\frac{s^{2}}{2^{9} \pi^{2} v^{4}}(1+\hat{x})^{-4} \hat{x}^{2} \leq \frac{1}{4}, \\
b_{0}^{W^{+} W^{-} \rightarrow h h h}(00)=\frac{s^{3}}{3 \times 2^{10} \pi^{4} v^{6}}(1+\hat{x})^{-7} \hat{x}^{4} \leq \frac{1}{4} .
\end{gathered}
$$

In the limit of small $\hat{x}$, VBF triple-Higgs boson production provides a weaker bound if $\sqrt{s} \geq \frac{16 \sqrt{3}}{3} v \approx 2.3 \mathrm{TeV}$.

TABLE X. Coefficients $\sigma_{j}^{h h h}$ (in fb) for different contributions to VBF $h h h$ in SILH with $c_{B}=-c_{W}$. We show values for $p p$ collisions at 14,27 , and $100 \mathrm{TeV}$, respectively.

\begin{tabular}{lccccccc}
\hline \hline$[\mathrm{fb}]$ & $\sigma_{\mathrm{SM}}^{h h h}=\sigma_{0}^{h h h}$ & $\sigma_{1}^{h h h}$ & $\sigma_{2}^{h h h}$ & $\sigma_{3}^{h h h}$ & $\sigma_{4}^{h h h}$ & $\sigma_{5}^{h h h}$ & $\sigma_{6}^{h h h}$ \\
\hline $14 \mathrm{TeV}$ & $2.792 \times 10^{-4}$ & $1.61 \times 10^{-3}$ & 1.89 & 0.198 & -3.85 & -0.218 & 2.09 \\
$27 \mathrm{TeV}$ & $1.66 \times 10^{-3}$ & $2.52 \times 10^{-3}$ & 30.1 & 1.87 & -58.3 & -1.97 & 29.6 \\
$100 \mathrm{TeV}$ & $3.10 \times 10^{-2}$ & -0.28 & $6.58 \times 10^{3}$ & 93.6 & $-1.22 \times 10^{4}$ & -93.2 & $5.87 \times 10^{3}$ \\
\hline \hline
\end{tabular}



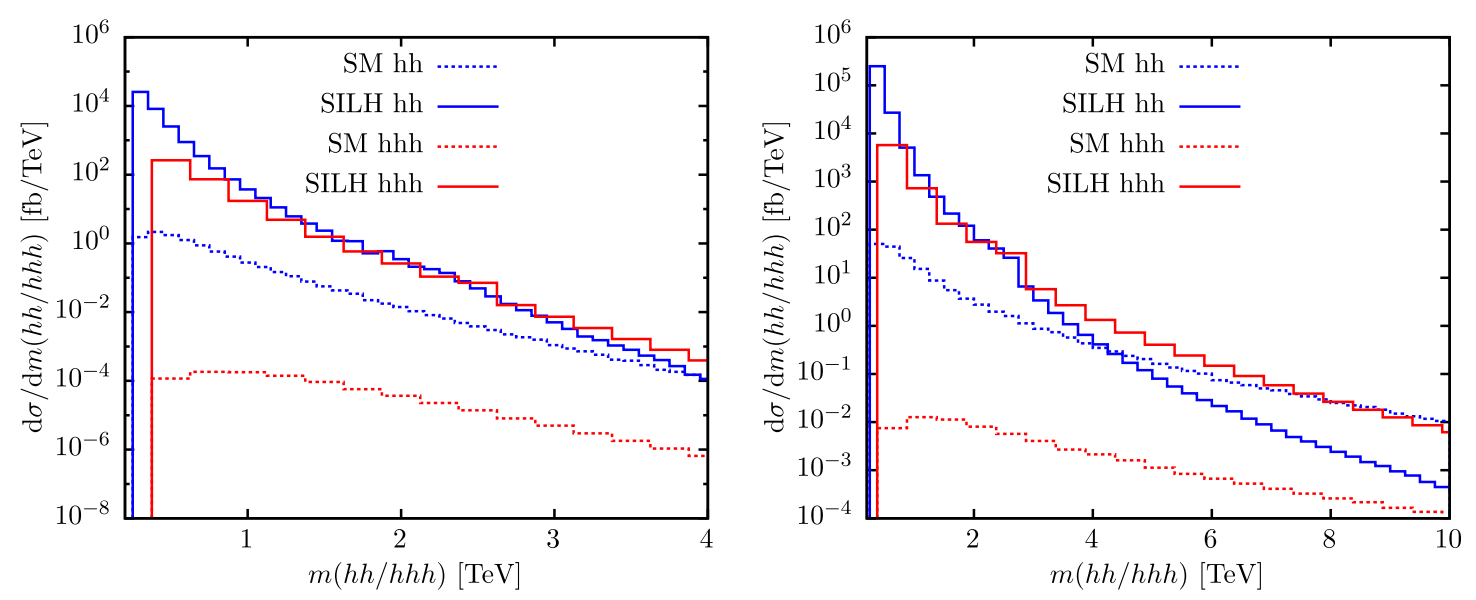

FIG. 14. Maximally allowed enhancement of the differential cross section of VBF $h h h$ production in $p p$ collisions in the SILH model, as a function of the triple-Higgs invariant mass. We display results for $14 \mathrm{TeV}$ (left) and for $100 \mathrm{TeV}$ (right), identifying $m(h h h)$ with the cutoff $Q$.

The cross sections for VBF double-Higgs and tripleHiggs production take the form

$$
\begin{gathered}
\sigma(h h)=(1+\hat{x})^{-6} \sum_{j=0}^{4} \sigma_{j}^{h h} \hat{x}^{j}, \\
\sigma(h h h)=(1+\hat{x})^{-9} \sum_{j=0}^{6} \sigma_{j}^{h h h} \hat{x}^{j} .
\end{gathered}
$$

We list the coefficients in Tables XI and XII, respectively. We can verify that the magnitude of the coefficients is consistent with Eqs. (4.16) and (4.17): $\left|\sigma_{2}^{h h}\right|,\left|\sigma_{3}^{h h}\right|,\left|\sigma_{4}^{h h}\right| \gg$ $\left|\sigma_{0}^{h h}\right|,\left|\sigma_{1}^{h h}\right|$ and $\left|\sigma_{4}^{h h h}\right|,\left|\sigma_{5}^{h h h}\right|,\left|\sigma_{6}^{h h h}\right| \gg\left|\sigma_{0}^{h h h}\right|,\left|\sigma_{1}^{h h h}\right|$, $\left|\sigma_{2}^{h h h}\right|,\left|\sigma_{3}^{h h h}\right|$.

In Fig. 15, we show the maximally allowed enhancement of the differential cross section in this restricted model. Again, the maximal deviations allowed by unitarity lift both
multi-Higgs processes by orders of magnitude, and the rates become comparable to each other. In contrast to the SILH case, the triple-production process approaches double production in the low-energy part of phase space, but falls off faster at high energy. This can be understood from Eqs. (4.16) and (4.17).

Comparing the unitarity bounds within the three models or parametrizations that we have considered, Figs. 7, 10, 14 , and 15 , we note that in the models with additional relations, there are regions where the $h h$ and $h h h$ cross sections become both large and of comparable magnitude. Individual bounds such as (4.18), (4.19) then have to be combined to a common bound according to (3.16). We should also account for multiple $W$ and $Z$ bosons in the final state. Since we have introduced the specific models for illustrative purposes, not expected to be complete or realistic in such an extreme parameter range, we did not attempt a more complete calculation. We expect combined bounds to be more restrictive, reduced by an effective

TABLE XI. Coefficients $\sigma_{j}^{h h h}$ (in fb) in Eq. (4.18). The values correspond to the Higgs-inflation model and apply to $p p$ collisions at 14,27 , and $100 \mathrm{TeV}$, respectively.

\begin{tabular}{lccccc}
\hline \hline$[\mathrm{fb}]$ & $\sigma_{\mathrm{SM}}^{h h}=\sigma_{0}^{h h}$ & $\sigma_{1}^{h h}$ & $\sigma_{2}^{h h}$ & $\sigma_{3}^{h h}$ & $\sigma_{4}^{h h}$ \\
\hline $14 \mathrm{TeV}$ & 1.10 & 8.07 & 33.7 & 47.1 & 20.3 \\
$27 \mathrm{TeV}$ & 4.32 & 33.6 & 154 & 223 & 98.9 \\
$100 \mathrm{TeV}$ & 41.2 & 331 & $2.20 \times 10^{3}$ & $3.57 \times 10^{3}$ & $1.64 \times 10^{3}$ \\
\hline \hline
\end{tabular}

TABLE XII. Coefficients $\sigma_{j}^{h h h}$ (in fb) in Eq. (4.19). The values correspond to the Higgs-inflation model and apply to $p p$ collisions at

\begin{tabular}{|c|c|c|c|c|c|c|c|}
\hline [fb] & $\sigma_{\mathrm{SM}}^{h h h}=\sigma_{0}^{h h h}$ & $\sigma_{1}^{h h h}$ & $\sigma_{2}^{h h h}$ & $\sigma_{3}^{h h h}$ & $\sigma_{4}^{h h h}$ & $\sigma_{5}^{h h h}$ & $\sigma_{6}^{h h h}$ \\
\hline $14 \mathrm{TeV}$ & $2.792 \times 10^{-4}$ & $3.72 \times 10^{-3}$ & $4.07 \times 10^{-2}$ & -0.132 & 2.49 & 5.51 & 2.86 \\
\hline $27 \mathrm{TeV}$ & $1.66 \times 10^{-3}$ & $2.47 \times 10^{-5}$ & 0.327 & -1.06 & 37.3 & 79.0 & 40.3 \\
\hline $100 \mathrm{TeV}$ & $3.10 \times 10^{-2}$ & 0.55 & 11.6 & -46.0 & $7.77 \times 10^{3}$ & $1.57 \times 10^{4}$ & $7.89 \times 10^{3}$ \\
\hline
\end{tabular}
14,27 , and $100 \mathrm{TeV}$, respectively. 

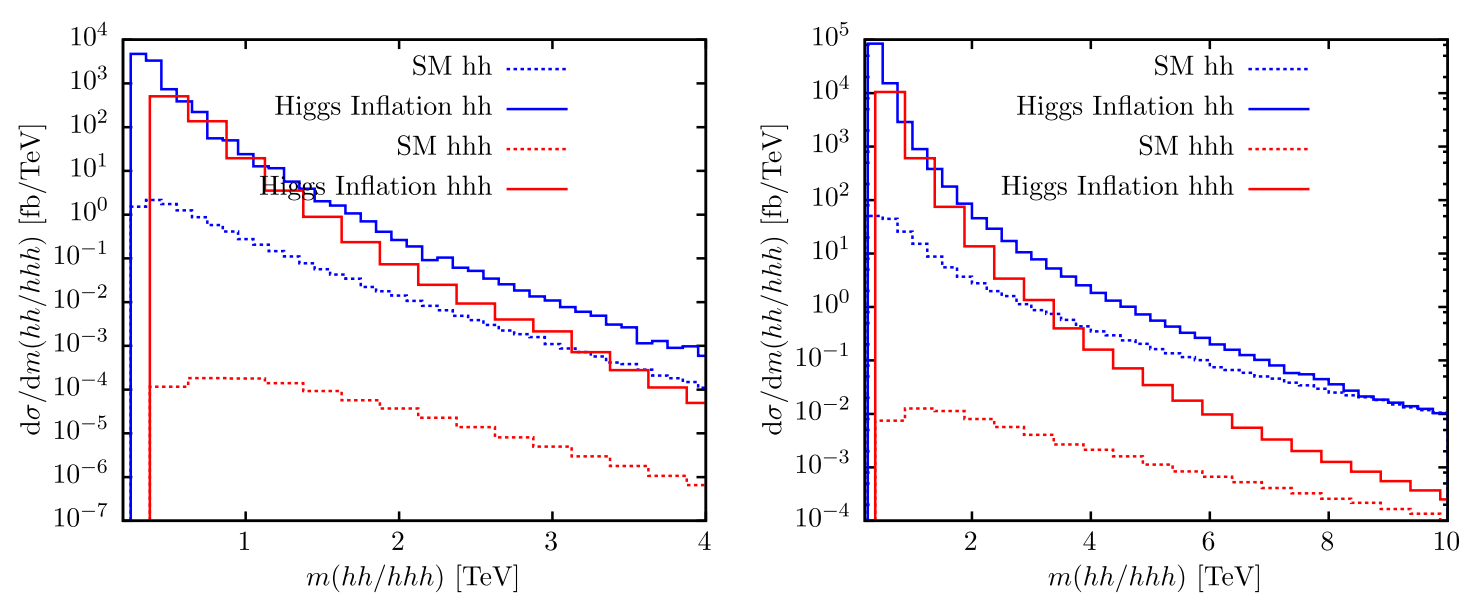

FIG. 15. Maximally allowed enhancement of the differential cross section of VBF $h$ hh production in $p p$ collisions in the Higgsinflation model, as a function of the triple-Higgs invariant mass. We display results for $14 \mathrm{TeV}$ (left) and for $100 \mathrm{TeV}$ (right), identifying $m(h h h)$ with the cutoff $Q$.

number of contributing final states which depends on the details of the model in the strongly interacting regime.

\section{SUMMARY AND DISCUSSION}

We have studied multi-Higgs boson production via VBF processes at the LHC and at future hadron colliders. While our emphasis lies on the rare triple-Higgs production mode, we have treated double- and triple-Higgs production processes in a common framework based on a generic Higgssector effective Lagrangian.

The generic effective Lagrangian given in Eq. (2.1) can be related to more restricted scenarios beyond the SM. Specifically, we have investigated two examples, the SILH model-linearly realized gauge symmetry, truncated at dimension six - and a Higgs-inflation model. Various further models with potentially strong interactions in the Higgs sector have been proposed in the literature, such as the minimal composite Higgs model [87], the composite twinHiggs model [88-90], or the composite minimal neutral naturalness model [91], cf. also [92]. Such models can be related to the generic effective Lagrangian in a similar way.

It is not surprising that even at a $100 \mathrm{TeV}$ proton-proton collider, observing the VBF multi-Higgs final state is very difficult and challenging if the SM is correct. Beyond the SM, models such as the ones mentioned above have revived the interest in new strong interactions with little impact on observables outside the Higgs sector, but potentially striking effects on the Higgs itself.

Anomalous interactions short of a new complete, weakly interacting theory, spoil the delicate gauge cancellations of the SM, leading to amplitudes which grow rapidly with energy and eventually saturate the unitarity limits. In this paper, we have derived unitarity limits for inelastic twoand three-particle production in the Higgs sector, and investigated their impact on the various possible contributions and form factors. It turns out that the unitarity limits are rather weak and allow for an enhancement of doubleand triple-Higgs production with rates that likely can be observed at a hadron collider, either the high-luminosity LHC or at a future high-energy $p p$ machine. In principle, the triple-Higgs rate can surpass the double-Higgs rate in part of the phase space, where the latter could also be enhanced by a large factor. We have computed the maximally possible enhancement in the context of either the generic EFT, or of the specific SILH and Higgs-inflation models.

For a more realistic study of the collider sensitivity to multi-Higgs production, one has to find strategies that enhance the signal in a difficult experimental environment, beyond the VBF cuts that we apply on the parton level. Regarding the theoretical models with which to compare, unitarity bounds have to be incorporated in a way that accounts for phase space in detail. There are generic algorithms such as in Refs. $[82,83,93]$, where the amplitude is projected onto the submanifold of amplitudes consistent with unitarity. Such a formalism should be applied to the case of inelastic and multiparticle production, to yield more specific limits, and proper matching to global-fit data obtained within the low-energy EFT. We defer this program to future work.

Our results confirm that despite the tiny rates for VBF multi-Higgs processes in the SM, substantial enhancements of multi-Higgs processes are a real possibility that is not in conflict with unitarity. Such final states should definitely be searched for in dedicated analyses.

\section{ACKNOWLEDGMENTS}

We thank Kaoru Hagiwara for useful discussions. W. K. thanks the CLICdp and Theory Groups for their hospitality at CERN, where part of this work was completed. W. K. is supported by the DFG Collaborative Research Center TRR 257 "Particle Physics Phenomenology after the Higgs Discovery." Z.Z. has been partially supported by a 
Nikolai Uraltsev Fellowship of the Center for Particle Physics, University of Siegen, and partially supported by the Natural Science Foundation of China under Grant No. 11875260 . S. S. is supported by the MOST of Taiwan under Grant No. 105-2811-M-002-130 and the CRF Grants of the Government of the Hong Kong SAR under HUKST4/CRF/13G. Q.-S. Y. is supported by the Natural Science Foundation of China under Grants No. 11475180 and No. 11875260. X.Z. has received funding from the European Union's Horizon 2020 research and innovation programme as part of the Marie SkłodowskaCurie Innovative Training Network MCnetITN3 (Grant No. 722104). We would like to acknowledge the Mainz Institute for Theoretical Physics for enabling us to complete this work.

\section{APPENDIX A: RELATION TO THE SILH EFFECTIVE LAGRANGIAN}

As mentioned above, the Lagrangian (2.1) does not manifestly exhibit electroweak gauge invariance. To reformulate it as an equivalent $S U(2)_{L} \times U(1)_{Y}$ invariant Lagrangian, we should apply the appropriate field redefinitions and connect our parameters to standard parametrizations in the literature. In fact, one may expect a linearly gauge-invariant Lagrangian to result from integrating out manifestly gaugeinvariant new physics at higher scales; for an example, cf. Ref. [94]. If we then truncate the gauge-invariant expansion at some fixed order, we obtain relations among the operator coefficients in (2.1).

In the following, we consider those relations for a particular version of the linear gauge representation truncated at dimension six, the SILH parametrization [70]. For the translation to different bases, cf., e.g., Refs. [87,95-97], these parameter relations are the consequence of truncating the gauge-invariant power-series expansion. By allowing higher-dimensional operators $(D \geq 8)$ in the EFT, we recover the original expression (2.1) after fixing the gauge.

There are various versions of the SILH effectiveLagrangian [70] parametrization. We refer to the following definition:

$$
\begin{aligned}
\mathcal{L}_{\mathrm{SILH}}= & \frac{c_{H}}{2 f^{2}} \partial^{\mu}\left(H^{\dagger} H\right) \partial_{\mu}\left(H^{\dagger} H\right)+\frac{c_{T}}{2 f^{2}}\left(H^{\dagger} \stackrel{\leftrightarrow}{D^{\mu}} H\right)\left(H^{\dagger} \stackrel{\leftrightarrow}{D}_{\mu} H\right)-\frac{c_{6} \lambda}{f^{2}}\left(H^{\dagger} H\right)^{3} \\
& +\left(\frac{c_{y} y_{f}}{f^{2}} H^{\dagger} H \bar{f}_{L} H f_{R}+\text { H.c. }\right)+\frac{c_{g} g_{S}^{2}}{16 \pi^{2} f^{2}} \frac{y_{t}^{2}}{g_{\rho}^{2}} H^{\dagger} H G_{\mu \nu}^{a} G^{a \mu \nu} \\
& +\frac{i c_{W} g}{2 m_{\rho}^{2}}\left(H^{\dagger} \sigma^{i} \stackrel{\leftrightarrow}{D^{\mu}} H\right)\left(D^{\nu} W_{\mu \nu}\right)^{i}+\frac{i c_{B} g^{\prime}}{2 m_{\rho}^{2}}\left(H^{\dagger} \stackrel{\leftrightarrow}{D^{\mu}} H\right)\left(\partial^{\nu} B_{\mu \nu}\right) \\
& +\frac{i c_{H W} g}{16 \pi^{2} f^{2}}\left(D^{\mu} H\right)^{\dagger} \sigma^{i}\left(D^{\nu} H\right) W_{\mu \nu}^{i}+\frac{i c_{H B} g^{\prime}}{16 \pi^{2} f^{2}}\left(D^{\mu} H\right)^{\dagger}\left(D^{\nu} H\right) B_{\mu \nu} \\
& +\frac{c_{\gamma} g^{\prime 2}}{16 \pi^{2} f^{2}} \frac{g^{2}}{g_{\rho}^{2}} H^{\dagger} H B_{\mu \nu} B^{\mu \nu} .
\end{aligned}
$$

Regarding derivatives acting on bosonic fields, we recall that we may apply the equations of motion

$$
\begin{gathered}
\left(D^{\mu} D_{\mu} H\right)^{j}=m^{2} H^{j}-\lambda\left(H^{\dagger} H\right) H^{j}-\bar{e} \Gamma_{e}^{\dagger} l^{j} \\
+\epsilon_{j k} \bar{q}^{k} \Gamma_{u} u-\bar{d} \Gamma_{d}^{\dagger} q^{j}, \\
\left(D^{\rho} G_{\rho \mu}\right)^{A}=g_{s}\left(\bar{q} \gamma_{\mu} T^{A} q+\bar{u} \gamma_{\mu} T^{A} u+\bar{d} \gamma_{\mu} T^{A} d\right), \\
\left(D^{\rho} W_{\rho \mu}\right)^{i}=\frac{g}{2}\left(H^{\dagger} i \stackrel{\leftrightarrow}{D_{\mu}^{i}} H+\bar{l} \gamma_{\mu} \tau^{i} l+\bar{q} \gamma_{\mu} \tau^{i} q\right), \\
\partial^{\rho} B_{\rho \mu}=g^{\prime} Y_{H} H^{\dagger} i \stackrel{\leftrightarrow}{i}{ }_{\mu} H+g^{\prime} \sum_{\psi \in\{l, e, q, u, d\}} Y_{\psi} \bar{\psi} \gamma_{\mu} \psi
\end{gathered}
$$

and trade them for terms with less derivatives and terms with flavor-diagonal contact interactions between bosons and fermions. Boson-fermion contact terms yield subleading effects in dedicated VBF data analyses, if we apply cuts that enhance the quasi on shell contribution for intermediate vector bosons, and optimize the analysis for resonant final-state vector bosons.

Further details of deriving the relation between the SILH Lagrangian and the effective Lagrangian (2.1), including conventions not listed here, are given in Appendix A 2 . Table II contains the actual translation between parameter sets.

We conclude this part with a remark on oblique corrections. According to Ref. [98], the parameter $\hat{S}$ is given by

$$
\begin{gathered}
\hat{S}=2 \frac{\cos \theta}{\sin \theta} c_{W B}, \\
\hat{T}=-c_{H},
\end{gathered}
$$

where $c_{W B} / v^{2} g g^{\prime}$ is the coefficient of the operator $H^{\dagger} \sigma^{i} H W_{\mu \nu}^{i} B^{\mu \nu} / g g^{\prime}$ for noncanonical gauge fields, and 
$c_{H}$ is the coefficient of the operator $\left|H^{\dagger} D_{\mu} H\right|^{2}$. If we translate the basis of Ref. [98] to our version of the SILH effective Lagrangian, we have

$$
\begin{gathered}
\hat{S}=-\left(c_{W}+c_{B}\right) \frac{m_{W}^{2}}{m_{\rho}^{2}}, \\
\hat{T}=-\frac{v^{2}}{f^{2}} c_{T} .
\end{gathered}
$$

We recall that $\hat{S}$ is constrained by data at the $10^{-3}$ level; the precise value depends on the variation of the other electroweak parameter $\hat{T}$. For our purposes, we have set $c_{T}$ to zero.

\section{Relation to models of Higgs inflation}

Higgs-inflation models [99-107] provide an interesting example of a scenario where new physics is associated with the Higgs sector, with little impact on other SM particles. Such models are notoriously difficult to identify, and any possible probe of Higgs interactions should be investigated. In the present context, this class of model provides an example of a scenario where the effective-Lagrangian description applies, the main effects are tied to the Higgs sector, and the parameter set is even more restricted. Conversely, the relations and limits that we derive for the parameters of the generic effective Lagrangian can be transferred to such a restricted model in a straightforward way.

We briefly review the derivation of the phenomenological Higgs Lagrangian for this model, where the Higgs field is coupled to gravity in a nonminimal way. The model is originally formulated as a Lagrangian in the Jordan frame,

$$
\begin{aligned}
S_{\text {Jordan }}= & \int \mathrm{d}^{4} x \sqrt{-g}\left\{-\frac{M^{2}+2 \xi H H^{\dagger}}{2}\right. \\
& \times R-\frac{1}{4} W^{a \mu \nu} W_{\mu \nu}^{a}-\frac{1}{4} B^{a \mu \nu} B_{\mu \nu}^{a} \\
& \left.+D_{\mu} H^{\dagger} D^{\mu} H-\lambda\left(H H^{\dagger}-\frac{v^{2}}{2}\right)^{2}\right\} .
\end{aligned}
$$

The value of $\xi$ can vary between $1 \ll \sqrt{\xi} \ll 10^{17}$, corresponding to $M \simeq M_{P}$.

For investigating the phenomenology, we apply the conformal transformation from the Jordan frame to the Einstein frame,

$$
\hat{g}_{\mu \nu}=\Omega^{2} g_{\mu \nu}, \quad \Omega^{2}=1+\frac{2 \xi H H^{\dagger}}{M_{P}^{2}} .
$$

This transformation leads to a nonminimal kinetic term for the Higgs field. In the unitary gauge $H=\frac{1}{\sqrt{2}}(0, h)^{T}$, we may introduce a scalar field $\chi$ as a transformed Higgs field,

$$
\mathrm{d} \chi=\sqrt{\frac{\Omega^{2}+12 \xi^{2} H H^{\dagger} / M_{P}^{2}}{\Omega^{4}}} \mathrm{~d} h .
$$

The action in the Einstein frame is

$S_{E} \supset \int d^{4} x \sqrt{-\hat{g}}\left\{-\frac{M_{P}^{2}}{2} \hat{R}+\partial_{\mu} \chi \partial^{\mu} \chi-U(\chi)\right\}$,

where $\hat{R}$ is calculated using the metric $\hat{g}_{\mu \nu}$. We neglect any renormalization-group running effect. The effective Higgs potential is

$$
U(\chi)=\frac{1}{\Omega(\chi)^{4}} \frac{\lambda}{4}\left(h(\chi)^{2}-\frac{v^{2}}{2}\right)^{2} .
$$

In the context of collider physics, we are looking at small field values $h \simeq \chi$ and $\Omega^{2} \simeq 1$, so the potential for the field $\chi$ is close to that of the initial Higgs field. Inflation physics is described by the large-field behavior of the Higgs field, the Higgs thus acting as an inflaton, where $h \gg M_{P} / \sqrt{\xi}$ (or $\left.\chi \gg \sqrt{6} M_{P}\right)$. In this range, we can approximate

$$
\begin{aligned}
h & \simeq \frac{M_{P}}{\sqrt{\xi}} \exp \left(\frac{\chi}{\sqrt{6} M_{P}}\right), \\
U(\chi) & =\frac{\lambda M_{P}^{4}}{4 \xi^{2}}\left(1+\exp \left(-\frac{2 \chi}{\sqrt{6} M_{P}}\right)\right)^{-2} .
\end{aligned}
$$

The potential is exponentially flat at large $h$, as appropriate for a model of inflation.

We are interested in collider phenomenology and thus assume small $h$ field values, so we replace $\chi$ by $h$ again. We plug Eq. (A12) into Eq. (A13) and omit higher-order terms. After reinstating the Higgs doublet notation $H$, we arrive $\mathrm{at}^{3}$

$$
\begin{aligned}
S_{E}= & \int d^{4} x \sqrt{-\hat{g}}\left\{-\frac{M_{P}^{2}}{2} \hat{R}+\right.\text { gauge interactions } \\
& +\frac{D_{\mu} H D^{\mu} H^{\dagger}}{\Omega^{2}}+\frac{3 \xi^{2}}{M_{p}^{2}} \frac{\partial_{\mu}\left(H^{\dagger} H\right) \partial^{\mu}\left(H H^{\dagger}\right)}{\Omega^{4}} \\
& -\frac{1}{\Omega^{2}} \lambda\left(H^{2}-\frac{v^{2}}{2}\right)^{2} \\
& \left.+\frac{2 H^{2}}{\Omega^{2}}\left(\frac{M_{W}^{2}}{v^{2}} W^{\mu} W_{\mu}+\frac{M_{Z}^{2}}{2 v^{2}} Z^{\mu} Z_{\mu}\right)\right\} .
\end{aligned}
$$

Details regarding the gauge interaction can be found in Ref. [108]. We obtain corrections to the coefficients of the following operators:

\footnotetext{
${ }^{3}$ Here we correct minor errors present in Ref. [58]. The corrections do not change the numerical results of that analysis.
} 


$$
\begin{aligned}
L_{V H}= & g_{W, a 1} \frac{2 m_{W}^{2}}{v} h W^{\mu} W_{\mu}+g_{W, a 2} \frac{m_{W}^{2}}{v^{2}} h^{2} W^{\mu} W_{\mu} \\
& +g_{W, a 3} \frac{m_{W}^{2}}{3 v^{3}} h^{3} W^{\mu} W_{\mu}+g_{Z, a 1} \frac{m_{Z}^{2}}{v} h Z^{\mu} Z_{\mu} \\
& +g_{Z, a 2} \frac{m_{Z}^{2}}{2 v^{2}} h^{2} Z^{\mu} Z_{\mu}+g_{Z, a 3} \frac{m_{Z}^{2}}{6 v^{3}} h^{3} Z^{\mu} Z_{\mu}+\cdots
\end{aligned}
$$

In Table II, we list the coefficient expressions for the Higgs-inflation model and relate them to the SILH operator basis and to the Higgs Lagrangian that we use for our study. It is evident that the SILH operator basis, which is appropriate for a generic strongly interacting model, incorporates directions in parameter space which are absent in the more specific model of Higgs inflation. Dedicated measurements of Higgs self-interactions become essential if such a class of model is realized.

\section{Relating the SILH parametrization to the Higgs EFT Lagrangian}

In our notation, the field strength tensors of the $U(1)$ and $S U(2)$ gauge groups are defined as

$$
\begin{gathered}
B_{\mu \nu}=\partial_{\mu} B_{\nu}-\partial_{\nu} B_{\mu}, \\
W_{\mu \nu}^{i}=\partial_{\mu} W_{\nu}^{i}-\partial_{\nu} W_{\mu}^{i}-g \epsilon^{i j k} W_{\mu}^{j} W_{\nu}^{k},
\end{gathered}
$$

respectively. The mass eigenstates of the gauge bosons are

$$
\begin{gathered}
B_{\mu}=\cos \theta A_{\mu}-\sin \theta Z_{\mu}, \\
W_{\mu}^{1}=\frac{1}{\sqrt{2}}\left(W_{\mu}^{-}+W_{\mu}^{+}\right), \\
W_{\mu}^{2}=\frac{1}{\sqrt{2}}\left(W_{\mu}^{-}-W_{\mu}^{+}\right), \\
W_{\mu}^{3}=\cos \theta Z_{\mu}+\sin \theta A_{\mu} .
\end{gathered}
$$

In unitary gauge, the Higgs doublet is given by

$$
H=\frac{1}{\sqrt{2}}\left(\begin{array}{c}
0 \\
v+h
\end{array}\right) .
$$

By using the equation of motion of $W_{\mu \nu}$ and $B_{\mu \nu}$ (cf. [97]),

$$
\begin{aligned}
& \left(D^{\rho} W_{\rho \mu}\right)^{i}=\frac{g}{2}\left(H^{\dagger} i \sigma^{i} \stackrel{\leftrightarrow}{D}_{\mu} H+\bar{l} \gamma_{\mu} \sigma^{i} l+\bar{q} \gamma_{\mu} \sigma^{i} q\right), \\
& \partial^{\rho} B_{\rho \mu}=g^{\prime} Y H^{\dagger} i \stackrel{\leftrightarrow}{D}_{\mu} H+g^{\prime} \sum_{\psi \in\{l, e, q, u, d\}} Y_{\psi} \bar{\psi} \gamma_{\mu} \psi,
\end{aligned}
$$

we obtain the following expressions for the operators with coefficients $c_{W}$ and $c_{B}$ :

$$
\begin{aligned}
\frac{i c_{W} g}{2 m_{\rho}^{2}}\left(H^{\dagger} \sigma^{i} \stackrel{\leftrightarrow}{D^{\mu}} H\right)\left(D^{\nu} W_{\mu \nu}\right)^{i} & =\frac{i c_{W}}{m_{\rho}^{2}} \frac{g^{2}}{4}\left(H^{\dagger} \sigma^{i} \stackrel{\leftrightarrow}{D^{\mu}} H\right)\left(-H^{\dagger} i \sigma^{i} \stackrel{\leftrightarrow}{D_{\mu}} H\right)+\ldots \\
=\frac{c_{W}}{m_{\rho}^{2}} \frac{g^{2}}{4} & {\left[-\frac{g^{2}}{4 \cos ^{2} \theta} Z^{\mu} Z_{\mu}(v+h)^{4}-\frac{g^{2}}{2} W^{+\mu} W_{\mu}^{-}(v+h)^{4}\right]+\ldots } \\
\frac{i c_{B} g^{\prime}}{2 m_{\rho}^{2}}\left(H^{\dagger} \stackrel{\leftrightarrow}{D^{\mu}} H\right)\left(\partial^{\nu} B_{\mu \nu}\right) & =\frac{i c_{B}}{m_{\rho}^{2}} \frac{g^{\prime 2}}{4}\left(H^{\dagger} \stackrel{\leftrightarrow}{D^{\mu}} H\right)\left(-H^{\dagger} i \stackrel{\leftrightarrow}{D_{\mu}} H\right)+\ldots \\
& =\frac{c_{B}}{m_{\rho}^{2}} \frac{g^{\prime 2}}{4}\left[-\frac{g^{2}}{4 \cos ^{2} \theta} Z^{\mu} Z_{\mu}(v+h)^{4}\right]+\ldots
\end{aligned}
$$

To obtain expressions for the operators with coefficients $c_{H W}$ and $c_{H B}$, we write the relations

$$
\begin{aligned}
2\left(D^{\mu} H\right)^{\dagger} \sigma^{i}\left(D^{\nu} H\right) W_{\mu \nu}^{i} & =H^{\dagger} \sigma^{i} \stackrel{\leftrightarrow}{D^{\mu}} H\left(D^{\nu} W_{\mu \nu}^{i}\right)-H^{\dagger} \sigma^{i}\left(D^{\mu} D^{\nu} H\right) W_{\mu \nu}^{i}-\left(D^{\nu} D^{\mu} H\right)^{\dagger} \sigma^{i} H W_{\mu \nu}^{i}+\text { total derivative } \\
= & H^{\dagger} \sigma^{i} \stackrel{\leftrightarrow}{D^{\mu}} H\left(D^{\nu} W_{\mu \nu}\right)^{i}+i \frac{g}{2} H^{\dagger} H W^{i \mu \nu} W_{\mu \nu}^{i}+i \frac{g^{\prime}}{2} H^{\dagger} \sigma^{i} H B^{\mu \nu} W_{\mu \nu}^{i}+\text { Total derivative, } \\
2\left(D^{\mu} H\right)^{\dagger}\left(D^{\nu} H\right) B_{\mu \nu} & =H^{\dagger} \stackrel{\leftrightarrow}{D^{\mu}} H\left(\partial^{\nu} B_{\mu \nu}\right)-H^{\dagger}\left(D^{\mu} D^{\nu} H\right) B_{\mu \nu}-\left(D^{\nu} D^{\mu} H\right)^{\dagger} H B_{\mu \nu}+\text { total derivative } \\
& =H^{\dagger} \stackrel{\leftrightarrow}{D^{\mu}} H \partial^{\nu} B_{\mu \nu}+i \frac{g}{2} H^{\dagger} \sigma^{i} H W^{i \mu \nu} B_{\mu \nu}+i \frac{g^{\prime}}{2} H^{\dagger} H B^{\mu \nu} B_{\mu \nu}+\text { Total derivative. }
\end{aligned}
$$

Here we used that $D^{\mu} D^{\nu}=\frac{1}{2}\left[D^{\mu}, D^{\nu}\right]+\frac{1}{2}\left\{D^{\mu}, D^{\nu}\right\}$ and $\left[D^{\mu}, D^{\nu}\right]=-i g \frac{\sigma^{i}}{2} W^{i \mu \nu}-i g^{\prime} Y B^{\mu \nu}$. Also, note that $\left\{D^{\mu}, D^{\nu}\right\}$ vanishes when it is being contracted with an antisymmetric tensor $W_{\mu \nu}, B_{\mu \nu}$. Equations (A29) and (A30) correspond to the analogous relations in Ref. [96]. Expanding in unitary gauge, we obtain 


$$
\begin{aligned}
\frac{i c_{H W} g}{16 \pi^{2} f^{2}}\left(D^{\mu} H\right)^{\dagger} \sigma^{i}\left(D^{\nu} H\right) W_{\mu \nu}^{i}= & \frac{i c_{H W} g}{16 \pi^{2} f^{2}}\left[\frac{1}{2}\left(H^{\dagger} \sigma^{i} \stackrel{\leftrightarrow}{D^{\mu}} H\right)\left(D^{\nu} W_{\mu \nu}\right)^{i}\right. \\
& \left.-i \frac{g}{4} H^{\dagger} H W_{\mu \nu}^{i} W^{i \mu \nu}-i \frac{g^{\prime}}{4} H^{\dagger} \sigma^{i} H W_{\mu \nu}^{i} B^{\mu \nu}\right] \\
= & \frac{c_{H W} g^{2}}{64 \pi^{2} f^{2}}\left[-\frac{m_{Z}^{2}}{v^{2}} Z^{\mu} Z_{\mu}(v+h)^{4}-\frac{2 m_{W}^{2}}{v^{2}} W^{+\mu} W_{\mu}^{-}(v+h)^{4}\right] \\
& +\frac{c_{H W} g^{2}}{128 \pi^{2} f^{2}}(v+h)^{2} W_{\mu \nu}^{i} W^{i \mu \nu}+\frac{c_{H W} g g^{\prime}}{64 \pi^{2} f^{2}} H^{\dagger} \sigma^{i} H W_{\mu \nu}^{i} B^{\mu \nu}+\ldots
\end{aligned}
$$

$$
\begin{aligned}
\frac{i c_{H B} g^{\prime}}{16 \pi^{2} f^{2}}\left(D^{\mu} H\right)^{\dagger}\left(D^{\nu} H\right) B_{\mu \nu} & =\frac{i c_{H B} g^{\prime}}{16 \pi^{2} f^{2}}\left[\frac{1}{2}\left(H^{\dagger} \stackrel{\leftrightarrow}{D^{\mu}} H\right)\left(\partial^{\nu} B_{\mu \nu}\right)-\frac{g^{\prime}}{4} H^{\dagger} H B_{\mu \nu} B^{\mu \nu}-i \frac{g}{4} H^{\dagger} \sigma^{i} H W_{\mu \nu}^{i} B^{\mu \nu}\right] \\
& =\frac{c_{H B} g^{\prime 2}}{64 \pi^{2} f^{2}}\left[-\frac{m_{Z}^{2}}{v^{2}} Z^{\mu} Z_{\mu}(v+h)^{4}\right]+\frac{c_{H B} g^{\prime 2}}{128 \pi^{2} f^{2}}(v+h)^{2} B_{\mu \nu} B^{\mu \nu}+\frac{c_{H B} g g^{\prime}}{64 \pi^{2} f^{2}} H^{\dagger} \sigma^{i} H W_{\mu \nu}^{i} B^{\mu \nu}+\ldots
\end{aligned}
$$

We arrive at the following kinetic part of the Lagrangian which includes the anomalous contributions:

$$
\begin{aligned}
\mathcal{L}_{\mathrm{kin}}= & \frac{1}{2}\left(1+c_{H} \frac{v^{2}}{f^{2}}\right) \partial^{\mu} h \partial_{\mu} h-\frac{1}{2}\left(1+c_{H W} \frac{g^{2} v^{2}}{32 \pi^{2} f^{2}}\right) W^{+\mu \nu} W_{\mu \nu}^{-} \\
& -\frac{1}{4}\left[1+\frac{g^{2} v^{2}}{32 \pi^{2} f^{2}}\left(c_{H W}+c_{H B} \tan ^{2} \theta-4 c_{\gamma} \frac{g^{\prime 2}}{g_{\rho}^{2}} \sin ^{2} \theta\right)\right] Z_{\mu \nu} Z^{\mu \nu} \\
& -\frac{1}{4}\left[1-\frac{g^{2} v^{2}}{32 \pi^{2} f^{2}}\left(4 c_{\gamma} \frac{g^{\prime 2}}{g_{\rho}^{2}} \cos ^{2} \theta\right)\right] A_{\mu \nu} A^{\mu \nu} \\
& -\frac{1}{4}\left[\frac{g g^{\prime} v^{2}}{32 \pi^{2} f^{2}}\left(c_{H W}-c_{H B}\right)+\frac{g g^{\prime} v^{2}}{16 \pi^{2} f^{2}}\left(4 c_{\gamma} \frac{g^{2}}{g_{\rho}^{2}} \sin ^{2} \theta\right)\right] Z_{\mu \nu} A^{\mu \nu} .
\end{aligned}
$$

The fields may be rescaled by

$$
\begin{gathered}
h=\left(1+c_{H} \frac{v^{2}}{f^{2}}\right)^{-\frac{1}{2}} h^{\prime}=\zeta_{h} h^{\prime}, \\
W_{\mu}^{ \pm}=\left(1+c_{H W} \frac{g^{2} v^{2}}{32 \pi^{2} f^{2}}\right)^{-\frac{1}{2}} W_{\mu}^{\prime \pm}=\zeta_{W} W_{\mu}^{\prime \pm}, \\
Z_{\mu}=\left[1+\frac{g^{2} v^{2}}{32 \pi^{2} f^{2}}\left(c_{H W}+c_{H B} \tan ^{2} \theta-4 c_{\gamma} \frac{g^{\prime 2}}{g_{\rho}^{2}} \sin ^{2} \theta\right)\right]^{-\frac{1}{2}} Z_{\mu}^{\prime}=\zeta_{Z}^{\prime} Z_{\mu}^{\prime}, \\
A_{\mu}=\left[1-\frac{g^{2} v^{2}}{32 \pi^{2} f^{2}}\left(4 c_{\gamma} \frac{g^{\prime 2}}{g_{\rho}^{2}} \cos ^{2} \theta\right)\right]^{-\frac{1}{2}} A_{\mu}^{\prime}=\zeta_{A} A_{\mu}^{\prime}
\end{gathered}
$$

to rewrite the Lagrangian in terms of normalized fields as

$$
\begin{aligned}
& \mathcal{L}_{\text {kin }}= \frac{1}{2} \partial^{\mu} h^{\prime} \partial_{\mu} h^{\prime}-\frac{1}{2} W^{\prime+\mu \nu} W_{\mu \nu}^{\prime-}-\frac{1}{4} Z_{\mu \nu}^{\prime} Z^{\prime \mu \nu}-\frac{1}{4} A_{\mu \nu}^{\prime} A^{\prime \mu \nu} \\
&-\frac{1}{4}\left[\frac{g g^{\prime} v^{2}}{32 \pi^{2} f^{2}}\left(c_{H W}-c_{H B}\right)+\frac{g g^{\prime} v^{2}}{16 \pi^{2} f^{2}}\left(4 c_{\gamma} \frac{g^{2}}{g_{\rho}^{2}} \sin ^{2} \theta\right)\right] \zeta_{A} \zeta_{Z}^{\prime} Z_{\mu \nu}^{\prime} A^{\prime \mu \nu}, \\
&=\frac{1}{2} \partial^{\mu} h^{\prime} \partial_{\mu} h^{\prime}-\frac{1}{2} W^{\prime+\mu \nu} W_{\mu \nu}^{\prime-}-\frac{1}{4} Z_{\mu \nu}^{\prime} Z^{\prime \mu \nu}-\frac{1}{4} A_{\mu \nu}^{\prime} A^{\prime \mu \nu}-\frac{1}{4} y_{Z A} \zeta_{A} \zeta_{Z}^{\prime} Z_{\mu \nu}^{\prime} A^{\prime \mu \nu} .
\end{aligned}
$$


To eliminate the $Z A$ mixing term, we introduce a linear shift as follows:

$$
\begin{gathered}
A_{\mu}^{\prime \prime}=A_{\mu}^{\prime}+y_{Z A} \zeta_{A} \zeta_{Z}^{\prime} Z_{\mu}^{\prime} / 2 \\
Z_{\mu}^{\prime \prime}=\sqrt{1+y_{Z A}^{2} \zeta_{A}^{2} \zeta_{Z}^{\prime 2} / 4 Z_{\mu}^{\prime}}=\zeta_{Z}^{-1} Z_{\mu} .
\end{gathered}
$$

This leads to

$$
A_{\mu}=\zeta_{A} A_{\mu}^{\prime \prime}-\frac{y_{Z A} \zeta_{A}^{2} \zeta_{Z}^{\prime}}{4} Z_{\mu}^{\prime \prime}=\zeta_{A} A_{\mu}^{\prime \prime}-\zeta_{A Z} Z_{\mu}^{\prime \prime}
$$

In the final result, all electroweak gauge bosons are canonically normalized, and we may omit the primes from the redefined fields. The factors $\zeta_{h}, \zeta_{W}, \zeta_{Z}, \zeta_{Z}^{\prime} \zeta_{A}$, and $\zeta_{A Z}$ are introduced for convenience.

From Eqs. (A27) and (A28), we also have the mass terms,

$$
\begin{aligned}
\mathcal{L}_{\text {mass }}= & -\lambda v^{2}\left(1+\frac{3}{2} c_{6} \frac{v^{2}}{f^{2}}\right) \zeta_{h}^{2} h^{2}+\frac{g^{2} v^{2}}{4}\left(1-c_{W} \frac{g^{2} v^{2}}{2 m_{\rho}^{2}}-c_{H W} \frac{g^{2} v^{2}}{32 \pi^{2} f^{2}}\right) \zeta_{W}^{2} W^{+\mu} W_{\mu}^{-} \\
& +\frac{g^{2} v^{2}}{8 \cos ^{2} \theta}\left(1-c_{W} \frac{g^{2} v^{2}}{2 m_{\rho}^{2}}-c_{B} \frac{g^{\prime 2} v^{2}}{2 m_{\rho}^{2}}-c_{H W} \frac{g^{2} v^{2}}{32 \pi^{2} f^{2}}-c_{H B} \frac{g^{\prime 2} v^{2}}{32 \pi^{2} f^{2}}\right) \zeta_{Z}^{2} Z_{\mu} Z^{\mu} .
\end{aligned}
$$

There are shifts in the $W$ and $Z$ mass given by

$$
\begin{gathered}
m_{W}^{2}=\frac{g^{2} v^{2}}{4}\left(1-c_{W} \frac{g^{2} v^{2}}{2 m_{\rho}^{2}}-c_{H W} \frac{g^{2} v^{2}}{32 \pi^{2} f^{2}}\right) \zeta_{W}^{2}, \\
m_{Z}^{2}=\frac{g^{2} v^{2}}{4 \cos ^{2} \theta}\left(1-c_{W} \frac{g^{2} v^{2}}{2 m_{\rho}^{2}}-c_{B} \frac{g^{2} v^{2}}{2 m_{\rho}^{2}}-c_{H W} \frac{g^{2} v^{2}}{32 \pi^{2} f^{2}}-c_{H B} \frac{g^{\prime 2} v^{2}}{32 \pi^{2} f^{2}}\right) \zeta_{Z}^{2} .
\end{gathered}
$$

After rescaling the fields, we read off the parameter relations that are listed in Table II.

\section{APPENDIX B: DETAILS FOR THE DERIVATION OF UNITARITY CONSTRAINTS}

\section{1. $2 \rightarrow 2$ scattering}

The application of unitarity conditions to elastic $2 \rightarrow 2$ scattering is well known. In this subsection, for completeness, we provide the explicit derivation and its connection to the generic formulas in Sec. III. The derivation is not restricted to elastic scattering; it applies to any combination of two-particle initial and final states $a$ and $b$, respectively.

For a two-particle state vector $\left|\alpha, \Phi_{a}\right\rangle$, working in the center of mass frame, it is convenient to choose the polar angle $\theta_{a}$ and azimuthal angle $\phi_{a}$ as phase-space parameters, or correspondingly, the normalized kinematics variables are $\vec{x}_{a}=\left(\frac{1}{2}\left(\cos \theta_{a}+1\right), \frac{\phi_{a}}{2 \pi}\right)$. The Jacobian determinant is given by

$$
J_{a}=\frac{1}{8 \pi} \frac{1}{S_{\alpha}} s^{-1} \sqrt{\left[s-\left(m_{a 1}+m_{a 2}\right)^{2}\right]\left[s-\left(m_{a 1}-m_{a 2}\right)^{2}\right]},
$$

where $m_{a 1}, m_{a 2}$ are the masses of particles in $a . S_{\alpha}$ is the symmetry factor that accounts for identical particles in $a$ with quantum-number combination $\alpha$ : if the two particles are identical then $S_{\alpha}=2$, otherwise $S_{\alpha}=1$.

Following Ref. [109], in the center of mass frame, the scattering matrix from the two-particle state $\left|\alpha, \Phi_{a}\right\rangle$ to $\left|\beta, \Phi_{b}\right\rangle$ can be expressed as follows ${ }^{4}$ :

$$
\begin{aligned}
M^{\beta \alpha}\left(x_{b}, x_{a}\right) \equiv & J_{b}^{\frac{1}{2}}\left\langle\beta, \theta_{b}, \phi_{b}|\mathcal{M}| \alpha, \theta_{a}, \phi_{a}\right\rangle J_{a}^{\frac{1}{2}} \\
= & 2 \sum_{j}(2 j+1) a_{j}^{\alpha \beta} D_{\lambda_{\alpha} \lambda_{\beta}}^{j}\left(\zeta_{1}, \zeta_{2}, \zeta_{3}\right) \\
= & 2 \sum_{j, m}(2 j+1) a_{j}^{\alpha \beta} D_{m \lambda_{\alpha}}^{j *}\left(\phi_{a}, \theta_{a}, 0\right) \\
& \times D_{m \lambda_{\beta}}^{j}\left(\phi_{b}, \theta_{b}, 0\right),
\end{aligned}
$$

where $\theta_{a}\left(\theta_{b}\right)$ are the polar angles and $\phi_{a}\left(\phi_{b}\right)$ are the azimuthal angles for the states $\left|\alpha, \Phi_{a}\right\rangle\left(\left|\beta, \Phi_{b}\right\rangle\right)$, respectively. $\zeta_{1}, \zeta_{2}, \zeta_{3}$ denote corresponding Euler angles which represent the rotation from direction $\left(\theta_{a}, \phi_{a}\right)$ to direction

\footnotetext{
${ }^{4}$ To be consistent with the explicit choice of polarization vector in Eq. (B6), our phase convention differs from Ref. [109].
} 
$\left(\theta_{b}, \phi_{b}\right)$. We use the standard convention for parametrizing four-momenta in terms of polar and azimuthal angles,

$$
p^{\mu}=(E,|\vec{p}| \sin \theta \cos \phi,|\vec{p}| \sin \theta \sin \phi,|\vec{p}| \cos \theta) .
$$

If the particle is a massive vector boson, we define the polarization states as follows:

$$
\begin{aligned}
&|p,+\rangle= \frac{1}{\sqrt{2}}(0, \cos \phi \cos \theta+i \sin \phi \\
&\sin \phi \cos \theta-i \cos \phi,-\sin \theta) \\
&|p,-\rangle= \frac{1}{\sqrt{2}}(0, \cos \phi \cos \theta-i \sin \phi \\
&\sin \phi \cos \theta+i \cos \phi,-\sin \theta), \\
&|p, 0\rangle=\left(\frac{|\vec{p}|}{m}, \frac{p^{0}}{m} \sin \theta \cos \phi, \frac{p^{0}}{m} \sin \theta \sin \phi, \frac{p^{0}}{m} \cos \theta\right),
\end{aligned}
$$

where $m=\sqrt{E^{2}-|\vec{p}|^{2}}$.

This expansion suggests that we choose the Wigner D matrix as an orthonormal basis for the two-particle phase space,

$$
H_{j m}^{\alpha}(\vec{x})=\sqrt{2 j+1} D_{m \lambda_{\alpha}}^{j *}\left(\phi_{a}, \theta_{a}, 0\right) .
$$

As a result, in the scattering amplitude between twoparticle states, the corresponding amplitude $a$ becomes diagonal and depends only on one index,

$$
a_{j m, j^{\prime} m^{\prime}}^{\alpha \beta}=\delta_{j j^{\prime}} \delta_{m m^{\prime}} a_{j}^{\alpha \beta},
$$

where we introduce reduced $a$ coefficients $a_{j}^{\alpha \beta}$.

Similarly, the $b$ coefficients can be reduced to a oneindex version,

$$
b_{j m}^{\alpha \beta}=\sum_{j^{\prime} m^{\prime}}\left|a_{j m, j^{\prime} m^{\prime}}^{\alpha \beta}\right|^{2}=\left|a_{j}^{\alpha \beta}\right|^{2} .
$$

The set of unitarity conditions (3.13) is thus reduced to

$$
\begin{gathered}
\left|\operatorname{Re} a_{j}^{\alpha \alpha}\right| \leq \frac{1}{2}, \\
\left|\operatorname{Im} a_{j}^{\alpha \alpha}-\frac{1}{2}\right| \leq \frac{1}{2}, \\
\sum_{\beta \neq \alpha} b_{j}^{\alpha \beta}=\sum_{\beta \neq \alpha}\left|a_{j}^{\alpha \beta}\right|^{2} \leq \frac{1}{4} .
\end{gathered}
$$

These conditions are equivalent to those in Refs. $[72,81$, 110 ], if only $2 \rightarrow 2$ processes are considered.

\section{2. $2 \rightarrow n$ scattering: general idea}

The unitarity conditions (3.13) do not depend on the characteristics of the intermediate state $c$, which may be any $n$-particle state. We have made use of this fact by expressing the conditions in terms of $b$ coefficients,

$$
\begin{aligned}
b_{A}^{\alpha \gamma} \equiv & \frac{1}{4} \int \mathrm{d} x_{a} \mathrm{~d} x_{b} \mathrm{~d} x_{c} H_{A}^{\alpha *}\left(x_{a}\right) H_{A}^{\alpha}\left(x_{b}\right) M^{\gamma \alpha *}\left(x_{c}, x_{b}\right) \\
& \times M^{\gamma \alpha}\left(x_{c}, x_{a}\right) \leq \frac{1}{4},
\end{aligned}
$$

which by construction are independent of the phase-space parametrization pertaining to $\Phi_{c}$. We keep the dependency on the discrete quantum numbers $\gamma$ of the intermediate state $c$.

In analogy to the $2 \rightarrow 2$ case above, we may use any orthonormal basis for the initial two-particle state $a$. Choosing the same Wigner D-matrix expansion is most convenient, since due to angular-momentum conservation, the $b$ coefficients only depend on one index,

$$
b_{j m}^{\alpha \gamma} \equiv b_{j m^{\prime}}^{\alpha \gamma} \equiv b_{j}^{\alpha \gamma},
$$

independent of the complexity of the intermediate states $c$. At this point, we may discuss the connection to previous literature on the subject $[73,74,76]$.

(i) In Refs. [73,74], unitarity constraints are formulated for the total cross section of $2 \rightarrow n$ scattering under the assumption that the $j=0$ partial wave ( $s$ wave) dominates. This assumption applies to some subset of the states that we consider here, but clearly is not justified for the generic case of polarized vectorboson scattering.

In fact, with our notation, the cross section for $a \rightarrow c$ with discrete quantum numbers $\alpha, \gamma$ is given by

$$
\begin{aligned}
\sigma_{\alpha \gamma}(a \rightarrow c)= & \frac{16 \pi S_{\alpha} s}{\left[s-\left(m_{a 1}+m_{a 2}\right)^{2}\right]\left[s-\left(m_{a 1}-m_{a 2}\right)^{2}\right]} \\
& \times \sum_{j}(2 j+1) b_{j}^{\alpha \gamma}, \quad(\mathrm{B} 15)
\end{aligned}
$$

where $b_{j}$ are the reduced $b$ coefficients after choosing the Wigner $\mathrm{D}$ matrix as basis.

Assuming that the $j=0$ partial wave dominates in the high-energy limit, we obtain

$$
\sigma_{\alpha \gamma}(a \rightarrow c) \approx \frac{16 \pi S_{\alpha}}{s} b_{0}^{\alpha \gamma} \leq \frac{4 \pi S_{\alpha}}{s},
$$

which is equivalent to the result of Refs. [73,74]. This inequality applies to any polarized cross section and could provide a stronger bound than its equivalent for an unpolarized cross section. 
(ii) Reference [76] considers the more generic situation of $2 \rightarrow n$ scattering without $s$-wave dominance, but restricts the derivation to spin-less particles. In that case, the Wigner D-matrix formalism collapses to the familiar formalism of Legendre polynomials and spherical harmonics. By the general relation,

$$
P_{l}\left(\cos \theta_{b a}\right)=\frac{4 \pi}{2 l+1} \sum_{m=-l}^{l} Y_{l}^{m}\left(\theta_{b}, \phi_{b}\right) Y_{l}^{m *}\left(\theta_{a}, \phi_{a}\right),
$$

the relative polar angle $\theta_{b a}$ can be determined via

$$
\cos \theta_{b a}=\cos \theta_{b} \cos \theta_{a}+\sin \theta_{b} \sin \theta_{a} \cos \left(\phi_{b}-\phi_{a}\right) .
$$

The Wigner D matrix reduces to spherical harmonics as follows:

$$
D_{m 0}^{j}(\phi, \theta, 0)=\sqrt{\frac{4 \pi}{2 j+1}} Y_{l}^{m *}(\theta, \phi) .
$$

With these relations, it is easy to verify that our formulas reduce to the ones of Ref. [76] in the spinless case.

\section{Generalized $s$ wave}

For some helicity combinations, the unitarity condition for $2 \rightarrow n$ scattering becomes independent of phase-space parameters in the high-energy limit. This is the situation which was considered in Refs. [73,74]. In this subsection, we work out the details for our application.

In the high-energy limit, effectively we can treat all external particles as massless, $p_{i}^{2}=0$. The generalized $s$-wave condition for scattering $a \rightarrow c$ takes the form

$$
\left\langle\gamma, \Phi_{c}|\mathcal{M}| \alpha, \Phi_{a}\right\rangle \approx \mathcal{C}
$$

where $\mathcal{C}$ is a constant with respect to the kinematical parameters, for fixed total four-momentum. In fact, in the EFT approximation, this situation occurs naturally for some of the terms since the leading contributions become polynomials of the Lorentz invariants.

(a) For the case of inelastic scattering $\alpha \neq \gamma$, the $b$ coefficients with (multi-)index $A$ take the form

$$
\begin{aligned}
b_{A}^{\alpha \gamma}= & \frac{1}{4} \int_{0}^{1} \mathrm{~d} \vec{x}_{a} \mathrm{~d}{\overrightarrow{x_{b}}}_{H_{A}^{\alpha *}}\left(\vec{x}_{a}\right) H_{A}^{\alpha}\left(\vec{x}_{b}\right) J_{\alpha}^{\frac{1}{2}}\left(\vec{x}_{a}\right) J_{\alpha}^{\frac{1}{2}}\left(\vec{x}_{b}\right) \\
& \times \int_{0}^{1} \mathrm{~d} \vec{x}_{c} J_{\gamma}\left(\vec{x}_{c}\right)\left\langle\gamma, \Phi_{c}|\mathcal{M}| \alpha, \Phi_{b}\right\rangle^{*}\left\langle\gamma, \Phi_{c}|\mathcal{M}| \alpha, \Phi_{a}\right\rangle \\
= & \frac{1}{4}|\mathcal{C}|^{2} \Delta_{\gamma}\left|F_{A}^{\alpha}\right|^{2}
\end{aligned}
$$

where the total phase-space volume $\Delta_{\gamma}$ is given by $[111,112]$

$$
\begin{aligned}
\Delta_{\gamma} \equiv & \int_{0}^{1} \mathrm{~d} \vec{x}_{c} J_{\gamma}\left(\vec{x}_{c}\right)=\frac{1}{S_{\gamma}} \frac{1}{(2 \pi)^{3 n_{\gamma}-4}}\left(\frac{\pi}{2}\right)^{n_{\gamma}-1} \\
& \times \frac{s^{n_{\gamma}-2}}{\left(n_{\gamma}-1\right) !\left(n_{\gamma}-2\right) !}
\end{aligned}
$$

and we define the function $F$ as

$$
F_{A}^{\alpha}=\int_{0}^{1} \mathrm{~d} \vec{x}_{a} H_{A}^{\alpha}\left(\vec{x}_{a}\right) J_{\alpha}^{\frac{1}{2}}\left(\vec{x}_{a}\right) .
$$

Using the Cauchy-Schwarz inequality, the orthonormality condition for the basis yields

$$
\left|F_{A}^{\alpha}\right|^{2} \leq \int_{0}^{1} \mathrm{~d} \vec{x}_{a}\left|H_{\vec{l}_{u}}^{\alpha}\left(\vec{x}_{a}\right)\right|^{2} \int_{0}^{1} \mathrm{~d} \vec{x}_{b} J_{\alpha}\left(\vec{x}_{b}\right)=\Delta_{\alpha}
$$

Therefore, we have

$$
b_{A}^{\alpha \gamma} \leq \frac{1}{4} \Delta_{\alpha} \Delta_{\gamma}|\mathcal{C}|^{2} .
$$

The strongest bound is obtained if the equals sign applies in Eq. (B25). The inequality becomes

$$
\frac{1}{4} \Delta_{\alpha} \Delta_{\gamma}|\mathcal{C}|^{2} \leq \frac{1}{4}
$$

To realize the optimal bound within a given phasespace parametrization, the following condition should be satisfied:

$$
\frac{H_{A}^{\alpha}\left(\vec{x}_{a}\right)}{J_{\alpha}^{\frac{1}{2}}\left(\vec{x}_{a}\right)}=\text { constant. }
$$

The condition can be met if $H_{A}^{\alpha}\left(\vec{x}_{a}\right)$ and $J_{\alpha}\left(\vec{x}_{a}\right)$ are both constant. Since a constant basis function is a member of commonly used orthonormal bases, the condition reduces to requiring a constant Jacobian determinant for the phase-space parametrization. For an algorithm which achieves this, cf. Ref. [112].

We observe that the bounds in Eq. (B26) are symmetric under the exchange $\alpha \leftrightarrow \gamma$, although the states $a$ and $c$ may differ in number or species of particles. We may exploit this property by performing polarization sums to either the initial or final state, when applying the formalism to scattering processes.

(b) In elastic scattering, i.e., $\alpha=\gamma$, the unitarity constraint may be expressed in terms of the $a$ coefficients rather than $b$ coefficients. After an analogous derivation, we arrive at the following optimal constraint: 


$$
\begin{gathered}
\left|\operatorname{Re} \frac{1}{2} \Delta_{\alpha} \mathcal{C}\right| \leq \frac{1}{2} \\
0 \leq\left|\operatorname{Im} \frac{1}{2} \Delta_{\alpha} \mathcal{C}-\frac{1}{4}\right| \leq 1
\end{gathered}
$$

The above discussion can be also applied to the case that the independence of phase-space parameters results from summing over degenerate states (polarization, color, etc.). Explicitly, for a set of degenerate states $S,^{5}$

$\sum_{\gamma \in S}\left(\left\langle\gamma, \Phi_{c}|\mathcal{M}| \alpha, \Phi_{a}\right\rangle\right)^{*}\left\langle\gamma, \Phi_{c}|\mathcal{M}| \alpha, \Phi_{b}\right\rangle=\left|\mathcal{C}_{S}\right|^{2}$,

where $\left|\mathcal{C}_{S}\right|^{2}$ is independent of the phase-space parameters $\vec{x}_{a}, \vec{x}_{b}, \vec{x}_{c}$. With an optimal choice of kinematic variables and basis, we obtain the bound

$$
\frac{1}{4} \Delta_{\alpha} \Delta_{\gamma}\left|\mathcal{C}_{S}\right|^{2} \leq \frac{1}{4}
$$

\section{Generic case: recursive kinematics}

For the concrete evaluation of unitarity bounds in the generic case where the phase-space parameter dependence remains nontrivial, we have to choose a specific phasespace parametrization. In our calculations, we used the standard recursive generation of $2 \rightarrow n$ phase space in terms of $2 \rightarrow 2$ scattering followed by a tree of $1 \rightarrow 2$ momentum splittings. The phase-space manifold ultimately is mapped to the $3 n-4$-dimensional unit hypercube, $\vec{x} \in[0,1]^{3 n-4}$. Below, we review this construction and provide the detailed formulas.

We denote the $n$-body phase-space element with total four-momentum $Q^{\mu}$ as $\mathrm{d} \Phi_{n}\left\{Q^{\mu}\right\}$.

(1) For $n>2$, this phase-space element is given by

$$
\begin{aligned}
& \mathrm{d} \Phi_{n}\left\{Q^{\mu}\right\}=\delta^{(4)}\left(\sum_{i=1}^{n} p_{i}^{\mu}-Q^{\mu}\right) \mathrm{d} \Phi_{n} \\
& =\frac{\mathrm{d}^{4} p_{n}}{(2 \pi)^{3}} \delta\left(p_{n}^{2}-m_{n}^{2}\right) \mathrm{d} \Phi_{n-1}\left\{Q^{\mu}-p_{n}^{\mu}\right\} .
\end{aligned}
$$

Working in the c.m. frame of $Q^{\mu}$ where $Q_{\mathrm{CM}}^{\mu}=\left(\sqrt{Q^{2}}, 0,0,0\right)$, we obtain

$$
\begin{aligned}
\frac{\mathrm{d}^{4} p_{n}}{(2 \pi)^{3}} \delta\left(p_{n}^{2}-m_{n}^{2}\right) & =\frac{\mathrm{d}^{4} p_{n, \mathrm{CM}}}{(2 \pi)^{3}} \delta\left(p_{n, \mathrm{CM}}^{2}-m_{n}^{2}\right) \\
& =\frac{\rho^{3}\left(Q^{2}, m_{n}, \sum_{i=1}^{n-1} m_{i}\right) x_{3 n-6}^{2} \sin \theta_{n}}{8 \pi E_{n, \mathrm{CM}}} \mathrm{d} x_{3 n-6} \mathrm{~d} x_{3 n-5} \mathrm{~d} x_{3 n-4},
\end{aligned}
$$

where the function $\rho$ is defined by

$$
\rho\left(s, m_{1}, m_{2}\right)=\sqrt{\left[s-\left(m_{1}+m_{2}\right)^{2}\right]\left[s-\left(m_{1}-m_{2}\right)^{2}\right]},
$$

and the four momentum $p_{n, \mathrm{CM}}$ satisfies

$$
\begin{gathered}
p_{n, \mathrm{CM}}^{\mu}=\left(E_{n, \mathrm{CM}}, \vec{p}_{n, \mathrm{CM}}\right), \\
\vec{p}_{n, \mathrm{CM}}=x_{3 n-6} \rho\left(Q^{2}, m_{n}, \sum_{i=1}^{n-1} m_{i}\right)\left(\sin \theta_{n} \cos \phi_{n}, \sin \theta_{n} \sin \phi_{n}, \cos \theta_{n}\right), \\
E_{n, \mathrm{CM}}=\sqrt{\left|\vec{p}_{n, \mathrm{CM}}\right|^{2}+m_{n}^{2}} \\
\theta_{n}=\pi x_{3 n-5}, \\
\phi_{n}=2 \pi x_{3 n-4} .
\end{gathered}
$$

The corresponding four momentum in original frame can be obtained by a simple Lorentz boost,

$$
\begin{aligned}
p_{n}^{\mu} & =\Lambda\left(Q_{\mathrm{CM}}, Q, p_{n, \mathrm{CM}}\right) \\
& =p_{n, \mathrm{CM}}^{\mu}-2\left(Q_{\mathrm{CM}}^{\mu}+Q^{\mu}\right) \frac{\left(Q_{\mathrm{CM}}+Q\right) \cdot p_{n, \mathrm{CM}}}{\left(Q_{\mathrm{CM}}+Q\right)^{2}}+2 Q^{\mu} \frac{Q_{\mathrm{CM}} \cdot p_{n, \mathrm{CM}}}{Q^{2}} .
\end{aligned}
$$

\footnotetext{
${ }^{5}$ We require all states in $S$ to have identical particle numbers and symmetry factors.
} 
(2) For $n=2$, working again in the c.m. frame, the formulas simplify accordingly,

$$
\mathrm{d} \Phi_{2}(Q)=\mathrm{d} x_{1} \mathrm{~d} x_{2} \frac{\rho\left(Q^{2}, m_{1}, m_{2}\right) \sin \theta}{128 \pi^{4} \sqrt{Q^{2}}},
$$

with

$$
\begin{gathered}
p_{1, \mathrm{CM}}^{\mu}=\left(\sqrt{p_{\mathrm{CM}}^{2}+m_{1}^{2}},-\vec{p}_{\mathrm{CM}}\right), \\
p_{2, \mathrm{CM}}^{\mu}=\left(\sqrt{p_{\mathrm{CM}}^{2}+m_{2}^{2}}, \vec{p}_{\mathrm{CM}}\right),
\end{gathered}
$$

$$
\begin{gathered}
\vec{p}_{\mathrm{CM}}=\rho\left(Q^{2}, m_{1}, m_{2}\right) \\
\times\left(\sin \theta_{2} \cos \phi_{2}, \sin \theta_{2} \sin \phi_{2}, \cos \theta_{2}\right), \\
\theta_{2}=\pi x_{1},
\end{gathered}
$$

$$
\phi_{2}=2 \pi x_{2} .
$$

Again, the corresponding four-momenta in the original frame can be obtained via the Lorentz boost given in Eq. (B.40).
[1] ATLAS Collaboration, Observation of a new particle in the search for the Standard Model Higgs boson with the ATLAS detector at the LHC, Phys. Lett. B 716, 1 (2012).

[2] CMS Collaboration, Observation of a new boson at a mass of $125 \mathrm{GeV}$ with the CMS experiment at the LHC, Phys. Lett. B 716, 30 (2012).

[3] CMS Collaboration, Search for Higgs boson pair production in the final state containing two photons and two bottom quarks in proton-proton collisions at $\sqrt{s}=13 \mathrm{TeV}$, Report No. CMS-PAS-HIG-17-008.

[4] T. Plehn, M. Spira, and P. M. Zerwas, Pair production of neutral Higgs particles in gluon-gluon collisions, Nucl. Phys. B479, 46 (1996).

[5] U. Baur, T. Plehn, and D. L. Rainwater, Measuring the Higgs Boson Self Coupling at the LHC and Finite Top Mass Matrix Elements, Phys. Rev. Lett. 89, 151801 (2002).

[6] Q. Li, Q.-S. Yan, and X. Zhao, Higgs pair production: Improved description by matrix element matching, Phys. Rev. D 89, 033015 (2014).

[7] B. Bhattacherjee and A. Choudhury, Role of supersymmetric heavy Higgs boson production in the self-coupling measurement of $125 \mathrm{GeV}$ Higgs boson at the LHC, Phys. Rev. D 91, 073015 (2015).

[8] Q.-H. Cao, B. Yan, D.-M. Zhang, and H. Zhang, Resolving the degeneracy in single Higgs production with Higgs pair production, Phys. Lett. B 752, 285 (2016).

[9] Q.-H. Cao, G. Li, B. Yan, D.-M. Zhang, and H. Zhang, Double Higgs production at the $14 \mathrm{TeV}$ LHC and a $100 \mathrm{TeV} p p$ collider, Phys. Rev. D 96, 095031 (2017).

[10] R. Grober, M. Muhlleitner, and M. Spira, Higgs pair production at NLO QCD for $C P$-violating Higgs sectors, Nucl. Phys. B925, 1 (2017).

[11] U. Baur, T. Plehn, and D. L. Rainwater, Determining the Higgs boson selfcoupling at hadron colliders, Phys. Rev. D 67, 033003 (2003).

[12] J. Ren, R.-Q. Xiao, M. Zhou, Y. Fang, H.-J. He, and W. Yao, LHC search of new Higgs boson via resonant DiHiggs production with decays into 4W, J. High Energy Phys. 06 (2018) 090.
[13] U. Baur, T. Plehn, and D. L. Rainwater, Probing the Higgs self-coupling at hadron colliders using rare decays, Phys. Rev. D 69, 053004 (2004).

[14] W. Yao, Studies of measuring Higgs self-coupling with $H H \rightarrow b \bar{b} \gamma \gamma$ at the future hadron colliders, arXiv: 1308.6302.

[15] F. Kling, T. Plehn, and P. Schichtel, Maximizing the significance in Higgs boson pair analyses, Phys. Rev. D 95, 035026 (2017).

[16] J. Chang, K. Cheung, J. S. Lee, C.-T. Lu, and J. Park, Higgs-boson-pair production $H(\rightarrow b \bar{b}) H(\rightarrow \gamma \gamma)$ from gluon fusion at the HL-LHC and HL-100 TeV hadron collider, Phys. Rev. D 100, 096001 (2019).

[17] J. H. Kim, Y. Sakaki, and M. Son, Combined analysis of double Higgs production via gluon fusion at the HL-LHC in the effective field theory approach, Phys. Rev. D 98, 015016 (2018).

[18] H.-J. He, J. Ren, and W. Yao, Probing new physics of cubic Higgs boson interaction via Higgs pair production at hadron colliders, Phys. Rev. D 93, 015003 (2016).

[19] A. Papaefstathiou, L. L. Yang, and J. Zurita, Higgs boson pair production at the LHC in the $b \bar{b} W^{+} W^{-}$channel, Phys. Rev. D 87, 011301 (2013).

[20] U. Baur, T. Plehn, and D. L. Rainwater, Examining the Higgs boson potential at lepton and hadron colliders: A comparative analysis, Phys. Rev. D 68, 033001 (2003).

[21] M. J. Dolan, C. Englert, and M. Spannowsky, Higgs selfcoupling measurements at the LHC, J. High Energy Phys. 10 (2012) 112.

[22] A. J. Barr, M. J. Dolan, C. Englert, and M. Spannowsky, Di-Higgs final states augMT2ed-selecting $h h$ events at the high luminosity LHC, Phys. Lett. B 728, 308 (2014).

[23] L.-C. L, C. Du, Y. Fang, H.-J. He, and H. Zhang, Searching heavier Higgs boson via di-Higgs production at LHC Run-2, Phys. Lett. B 755, 509 (2016).

[24] D. E. Ferreira de Lima, A. Papaefstathiou, and M. Spannowsky, Standard model Higgs boson pair production in the $(b \bar{b})(b \bar{b})$ final state, J. High Energy Phys. 08 (2014) 030 . 
[25] J. K. Behr, D. Bortoletto, J. A. Frost, N. P. Hartland, C. Issever, and J. Rojo, Boosting Higgs pair production in the $b \bar{b} b \bar{b}$ final state with multivariate techniques, Eur. Phys. J. C 76, 386 (2016).

[26] V. Barger, L. L. Everett, C. B. Jackson, and G. Shaughnessy, Higgs-pair production and measurement of the triscalar coupling at $\operatorname{LHC}(8,14)$, Phys. Lett. B 728, 433 (2014).

[27] A. J. Barr, M. J. Dolan, C. Englert, D. E. Ferreira de Lima, and M. Spannowsky, Higgs self-coupling measurements at a 100 TeV hadron collider, J. High Energy Phys. 02 (2015) 016.

[28] A. Papaefstathiou, Discovering Higgs boson pair production through rare final states at a $100 \mathrm{TeV}$ collider, Phys. Rev. D 91, 113016 (2015).

[29] Q. Li, Z. Li, Q.-S. Yan, and X. Zhao, Probe Higgs boson pair production via the $3 \ell 2 j+\mathbb{E}$ mode, Phys. Rev. D 92, 014015 (2015).

[30] X. Zhao, Q. Li, Z. Li, and Q.-S. Yan, Discovery potential of Higgs boson pair production through $4 \ell+\mathbb{E}$ final states at a $100 \mathrm{TeV}$ collider, Chin. Phys. C 41, 023105 (2017).

[31] R. Contino et al., Physics at a $100 \mathrm{TeV}$ pp collider: Higgs and EW symmetry breaking studies, CERN Yellow Rep. 3, 255 (2017).

[32] D. Gonalves, T. Han, F. Kling, T. Plehn, and M. Takeuchi, Higgs boson pair production at future hadron colliders: From kinematics to dynamics, Phys. Rev. D 97, 113004 (2018).

[33] D. R. T. Jones and S. T. Petcov, Heavy higgs bosons at LEP, Phys. Lett. 84B, 440 (1979).

[34] ATLAS Collaboration, Measurement of the Higgs boson coupling properties in the $H \rightarrow Z Z^{*} \rightarrow 4 \ell$ decay channel at $\sqrt{s}=13 \mathrm{TeV}$ with the ATLAS detector, Report No. ATLAS-CONF-2017-043.

[35] CMS Collaboration, Higgs to WW measurements with $15.2 \mathrm{fb}^{-1}$ of $13 \mathrm{TeV}$ proton-proton collisions, Report No. CMS-PAS-HIG-16-021.

[36] M. J. Dolan, C. Englert, N. Greiner, and M. Spannowsky, Further on up the Road: $h h j j$ Production at the LHC, Phys. Rev. Lett. 112, 101802 (2014).

[37] L.-S. Ling, R.-Y. Zhang, W.-G. Ma, L. Guo, W.-H. Li, and X.-Z. Li, NNLO QCD corrections to Higgs pair production via vector boson fusion at hadron colliders, Phys. Rev. D 89, 073001 (2014).

[38] M. J. Dolan, C. Englert, N. Greiner, K. Nordstrom, and M. Spannowsky, $h h j j$ production at the LHC, Eur. Phys. J. C 75, 387 (2015).

[39] F. Bishara, R. Contino, and J. Rojo, Higgs pair production in vector-boson fusion at the LHC and beyond, Eur. Phys. J. C 77, 481 (2017).

[40] E. Arganda, C. Garcia-Garcia, and M. J. Herrero, Probing the Higgs self-coupling through double Higgs production in vector boson scattering at the LHC, Nucl. Phys. B945, 114687 (2019).

[41] J. Baglio, A. Djouadi, R. Grber, M. M. Mhlleitner, J. Quevillon, and M. Spira, The measurement of the Higgs self-coupling at the LHC: Theoretical status, J. High Energy Phys. 04 (2013) 151.

[42] R. Frederix, S. Frixione, V. Hirschi, F. Maltoni, O. Mattelaer, P. Torrielli, E. Vryonidou, and M. Zaro, Higgs pair production at the LHC with NLO and parton-shower effects, Phys. Lett. B 732, 142 (2014).

[43] C. Englert, Q. Li, M. Spannowsky, M. Wang, and L. Wang, VBS $\mathrm{W}^{ \pm} \mathrm{W}^{ \pm} \mathrm{H}$ production at the HL-LHC and a $100 \mathrm{TeV} p$-collider, Int. J. Mod. Phys. A 32, 1750106 (2017).

[44] M. Moretti, S. Moretti, F. Piccinini, R. Pittau, and A. D. Polosa, Higgs boson self-couplings at the LHC as a probe of extended Higgs sectors, J. High Energy Phys. 02 (2005) 024.

[45] C. Englert, F. Krauss, M. Spannowsky, and J. Thompson, Di-Higgs phenomenology in $t \bar{t} h h$ : The forgotten channel, Phys. Lett. B 743, 93 (2015).

[46] T. Liu and H. Zhang, Measuring Di-Higgs physics via the $t \bar{t} h h \rightarrow t \bar{t} b \bar{b} b \bar{b}$ channel, arXiv:1410.1855.

[47] Q.-H. Cao, Y. Liu, and B. Yan, Measuring trilinear Higgs coupling in $\mathrm{WHH}$ and $\mathrm{ZHH}$ productions at the high-luminosity LHC, Phys. Rev. D 95, 073006 (2017).

[48] V. D. Barger, T. Han, and R. J. N. Phillips, Double Higgs boson bremsstrahlung from $W$ and $Z$ bosons at supercolliders, Phys. Rev. D 38, 2766 (1988).

[49] H. T. Li and J. Wang, Fully differential Higgs pair production in association with a $W$ boson at next-tonext-to-leading order in QCD, Phys. Lett. B 765, 265 (2017).

[50] K. Nordstrm and A. Papaefstathiou, $V H H$ production at the high-luminosity LHC, Eur. Phys. J. Plus 134, 288 (2019).

[51] T. Plehn and M. Rauch, The quartic Higgs coupling at hadron colliders, Phys. Rev. D 72, 053008 (2005).

[52] T. Binoth, S. Karg, N. Kauer, and R. Ruckl, Multi-Higgs boson production in the Standard Model and beyond, Phys. Rev. D 74, 113008 (2006).

[53] D. A. Dicus, C. Kao, and W. W. Repko, Self-coupling of the Higgs boson in the processes $p p \rightarrow Z H H H+X$ and $p p \rightarrow W H H H+X$, Phys. Rev. D 93, 113003 (2016).

[54] F. Maltoni, E. Vryonidou, and M. Zaro, Top-quark mass effects in double and triple Higgs production in gluongluon fusion at NLO, J. High Energy Phys. 11 (2014) 079.

[55] A. Papaefstathiou and K. Sakurai, Triple Higgs boson production at a $100 \mathrm{TeV}$ proton-proton collider, J. High Energy Phys. 02 (2016) 006.

[56] C.-Y. Chen, Q.-S. Yan, X. Zhao, Y.-M. Zhong, and Z. Zhao, Probing triple-Higgs productions via $4 b 2 \gamma$ decay channel at a $100 \mathrm{TeV}$ hadron collider, Phys. Rev. D 93, 013007 (2016).

[57] B. Fuks, J.H. Kim, and S. J. Lee, Probing Higgs self-interactions in proton-proton collisions at a centerof-mass energy of $100 \mathrm{TeV}$, Phys. Rev. D 93, 035026 (2016).

[58] W. Kilian, S. Sun, Q.-S. Yan, X. Zhao, and Z. Zhao, New physics in multi-Higgs boson final states, J. High Energy Phys. 06 (2017) 145.

[59] P. Agrawal, D. Saha, and A. Shivaji, Production of $H H H$ and $H H V(V=\gamma, Z)$ at the hadron colliders, Phys. Rev. D 97, 036006 (2018).

[60] B. Fuks, J.H. Kim, and S. J. Lee, Scrutinizing the Higgs quartic coupling at a future $100 \mathrm{TeV}$ protonproton collider with taus and b-jets, Phys. Lett. B 771, 354 (2017). 
[61] R. Contino, C. Grojean, D. Pappadopulo, R. Rattazzi, and A. Thamm, Strong Higgs interactions at a linear collider, J. High Energy Phys. 02 (2014) 006.

[62] A. S. Belyaev, P. B. Schaefers, and M. C. Thomas, Precise test of Higgs boson properties via triple Higgs boson production in vector boson fusion at future colliders, Phys. Rev. D 99, 015030 (2019).

[63] ILD Collaboration, Measurement of Higgs couplings and self-coupling at the ILC, Proc. Sci., EPS-HEP2013 (2013) 316 [arXiv:1311.6528].

[64] H. Abramowicz et al., Higgs physics at the CLIC electronpositron linear collider, Eur. Phys. J. C 77, 475 (2017).

[65] A. Djouadi, W. Kilian, M. Muhlleitner, and P. M. Zerwas, Testing Higgs self-couplings at $e+e-$ linear colliders, Eur. Phys. J. C 10, 27 (1999).

[66] F. Maltoni, D. Pagani, and X. Zhao, Constraining the Higgs self-couplings at $e^{+} e^{-}$colliders, J. High Energy Phys. 07 (2018) 087.

[67] W. Kilian, T. Ohl, and J. Reuter, WhIZARD: Simulating multi-particle processes at LHC and ILC, Eur. Phys. J. C 71, 1742 (2011).

[68] J. Alwall, R. Frederix, S. Frixione, V. Hirschi, F. Maltoni, O. Mattelaer, H.-S. Shao, T. Stelzer, P. Torrielli, and M. Zaro, The automated computation of tree-level and nextto-leading order differential cross sections, and their matching to parton shower simulations, J. High Energy Phys. 07 (2014) 079.

[69] R. Alonso, E. E. Jenkins, and A. V. Manohar, Geometry of the scalar sector, J. High Energy Phys. 08 (2016) 101.

[70] G. F. Giudice, C. Grojean, A. Pomarol, and R. Rattazzi, The strongly-interacting light Higgs, J. High Energy Phys. 06 (2007) 045.

[71] J. M. Cornwall, D. N. Levin, and G. Tiktopoulos, Derivation of gauge invariance from high-energy unitarity bounds on the s matrix, Phys. Rev. D 10, 1145 (1974).

[72] U. Baur and D. Zeppenfeld, Unitarity constraints on the electroweak three vector boson vertices, Phys. Lett. B 201, 383 (1988).

[73] F. Maltoni, J. M. Niczyporuk, and S. Willenbrock, The scale of fermion mass generation, Phys. Rev. D 65, 033004 (2002).

[74] D. A. Dicus and H.-J. He, Scales of fermion mass generation and electroweak symmetry breaking, Phys. Rev. D 71, 093009 (2005).

[75] D. A. Dicus and H.-J. He, Scales of Mass Generation for Quarks, Leptons and Majorana Neutrinos, Phys. Rev. Lett. 94, 221802 (2005).

[76] Z.-H. Yu, Q.-S. Yan, and P.-F. Yin, Detecting interactions between dark matter and photons at high energy $e^{+} e^{-}$ colliders, Phys. Rev. D 88, 075015 (2013).

[77] T. Kinoshita, Mass singularities of Feynman amplitudes, J. Math. Phys. (N.Y.) 3, 650 (1962).

[78] T. D. Lee and M. Nauenberg, Degenerate systems and mass singularities, Phys. Rev. 133, B1549 (1964).

[79] R. E. Cutkosky, Singularities and discontinuities of Feynman amplitudes, J. Math. Phys. (N.Y.) 1, 429 (1960).

[80] M. J. G. Veltman, Unitarity and causality in a renormalizable field theory with unstable particles, Physica (Amsterdam) 29, 186 (1963).
[81] T. Corbett, O. J. P. boli, and M. C. Gonzalez-Garcia, Unitarity constraints on dimension-six operators, Phys. Rev. D 91, 035014 (2015).

[82] G. Perez, M. Sekulla, and D. Zeppenfeld, Anomalous quartic gauge couplings and unitarization for the vector boson scattering process $p p \rightarrow W^{+} W^{+} j j X \rightarrow$ $\ell^{+} \nu_{\ell} \ell^{+} \nu_{\ell} j j X$, Eur. Phys. J. C 78, 759 (2018).

[83] S. Brass, C. Fleper, W. Kilian, J. Reuter, and M. Sekulla, Transversal modes and Higgs bosons in electroweak vector-boson scattering at the LHC, Eur. Phys. J. C 78, 931 (2018).

[84] J. Baglio et al., Release note-VBFNLO 2.7.0, arXiv: 1404.3940.

[85] J. Baglio et al., VBFNLO: A parton level Monte Carlo for processes with electroweak bosons-Manual for version 2.7.0, arXiv:1107.4038.

[86] S. Dawson, The effective $W$ approximation, Nucl. Phys. B249, 42 (1985).

[87] K. Agashe, R. Contino, and A. Pomarol, The minimal composite Higgs model, Nucl. Phys. B719, 165 (2005).

[88] M. Geller and O. Telem, Holographic Twin Higgs Model, Phys. Rev. Lett. 114, 191801 (2015).

[89] R. Barbieri, D. Greco, R. Rattazzi, and A. Wulzer, The composite twin Higgs scenario, J. High Energy Phys. 08 (2015) 161.

[90] M. Low, A. Tesi, and L.-T. Wang, Twin Higgs mechanism and a composite Higgs boson, Phys. Rev. D 91, 095012 (2015).

[91] L.-X. Xu, J.-H. Yu, and S.-H. Zhu, Minimal neutral naturalness model, arXiv:1810.01882.

[92] H.-L. Li, L.-X. Xu, J.-H. Yu, and S.-H. Zhu, EFTs meet Higgs nonlinearity, compositeness and (Neutral) naturalness, J. High Energy Phys. 09 (2019) 010.

[93] W. Kilian, T. Ohl, J. Reuter, and M. Sekulla, High-energy vector boson scattering after the Higgs discovery, Phys. Rev. D 91, 096007 (2015).

[94] T. Corbett, A. Joglekar, H.-L. Li, and J.-H. Yu, Exploring extended scalar sectors with Di-Higgs signals: A Higgs EFT perspective, J. High Energy Phys. 05 (2018) 061.

[95] D. B. Kaplan and H. Georgi, SU(2) $\times \mathrm{U}(1)$ breaking by vacuum misalignment, Phys. Lett. B 136B, 183 (1984).

[96] R. Contino, M. Ghezzi, C. Grojean, M. Muhlleitner, and M. Spira, Effective Lagrangian for a light Higgs-like scalar, J. High Energy Phys. 07 (2013) 035.

[97] B. Grzadkowski, M. Iskrzynski, M. Misiak, and J. Rosiek, Dimension-six terms in the standard model lagrangian, J. High Energy Phys. 10 (2010) 085.

[98] R. Barbieri, A. Pomarol, R. Rattazzi, and A. Strumia, Electroweak symmetry breaking after LEP-1 and LEP-2, Nucl. Phys. B703, 127 (2004).

[99] F. L. Bezrukov and M. Shaposhnikov, The Standard Model Higgs boson as the inflaton, Phys. Lett. B 659, 703 (2008).

[100] F. Bezrukov and M. Shaposhnikov, Standard Model Higgs boson mass from inflation: Two loop analysis, J. High Energy Phys. 07 (2009) 089.

[101] Y. Hamada, T. Noumi, S. Sun, and G. Shiu, An O (750) $\mathrm{GeV}$ resonance and inflation, Phys. Rev. D 93, 123514 (2016). 
[102] J. Ren, Z.-Z. Xianyu, and H.-J. He, Higgs gravitational interaction, weak boson scattering, and Higgs inflation in Jordan and Einstein frames, J. Cosmol. Astropart. Phys. 06 (2014) 032.

[103] H.-J. He and Z.-Z. Xianyu, Extending Higgs inflation with $\mathrm{TeV}$ scale new physics, J. Cosmol. Astropart. Phys. 10 (2014) 019.

[104] Z.-Z. Xianyu and H.-J. He, Asymptotically safe Higgs inflation, J. Cosmol. Astropart. Phys. 10 (2014) 083.

[105] S.-F. Ge, H.-J. He, J. Ren, and Z.-Z. Xianyu, Realizing dark matter and Higgs inflation in light of LHC diphoton excess, Phys. Lett. B 757, 480 (2016).

[106] J. Ellis, H.-J. He, and Z.-Z. Xianyu, Higgs inflation, reheating and gravitino production in no-scale supersymmetric GUTs, J. Cosmol. Astropart. Phys. 08 (2016) 068.
[107] J. Ellis, H.-J. He, and Z.-Z. Xianyu, New Higgs inflation in a no-scale supersymmetric SU(5) GUT, Phys. Rev. D 91, 021302 (2015).

[108] I. Obata, T. Miura, and J. Soda, Dynamics of electroweak gauge fields during and after Higgs inflation, Phys. Rev. D 90, 045005 (2014).

[109] M. Jacob and G.C. Wick, On the general theory of collisions for particles with spin, Ann. Phys. (N.Y.) 7, 404 (1959).

[110] M. Dahiya, S. Dutta, and R. Islam, Investigating perturbative unitarity in the presence of anomalous couplings, Phys. Rev. D 93, 055013 (2016).

[111] R. Kleiss, W. J. Stirling, and S.D. Ellis, A new Monte Carlo treatment of multiparticle phase space at high-energies, Comput. Phys. Commun. 40, 359 (1986).

[112] S. Pltzer, RAMBO on diet, arXiv:1308.2922. 